\title{
Strong convergence rates for nonlinearity-truncated Euler-type approximations of stochastic Ginzburg-Landau equations
}

\author{
Sebastian Becker and Arnulf Jentzen
}

January 22, 2016

\begin{abstract}
This article proposes and analyzes explicit and easily implementable temporal numerical approximation schemes for additive noise-driven stochastic partial differential equations (SPDEs) with polynomial nonlinearities such as, e.g., stochastic Ginzburg-Landau equations. We prove essentially sharp strong convergence rates for the considered approximation schemes. Our analysis is carried out for abstract stochastic evolution equations on separable Banach and Hilbert spaces including the above mentioned SPDEs as special cases. We also illustrate our strong convergence rate results by means of a numerical simulation in MATLAB.
\end{abstract}

\section{Contents}

1 Introduction

1.1 Notation . . . . . . . . . . . . . . . . . . . . . . 7

2 A priori bounds

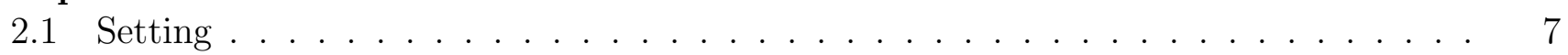

2.2 A priori bounds based on variational arguments . . . . . . . . . . . . . . . . . 7

2.3 A priori bounds based on bootstrap-type arguments . . . . . . . . . . . . . 8

3 Pathwise error estimates $\quad 10$

3.1 Setting . . . . . . . . . . . . . . . . . . . . . . 10

3.2 Regularity of the exact solution . . . . . . . . . . . . . . . . 10

3.3 Regularity of the semilinear integrated version of the numerical approximation . . . 15

3.4 Analysis of the error between the numerical approximation and its semilinear integrated version . . . . . . . . . . . . . . . . . . 20

3.5 Analysis of the error between the exact solution and the semilinear integrated version of the numerical approximation . . . . . . . . . . . . . . . . . 
4.1 Setting . . . . . . . . . . . . . . . . . . . . . . 23

4.2 Analysis of the error between the numerical approximation and its semilinear integrated version . . . . . . . . . . . . . . . . .

4.3 Analysis of the error between the exact solution and the semilinear integrated version of the numerical approximation . . . . . . . . . . . . . .

4.4 Analysis of the error between the exact solution and the numerical approximation .

5 Main result

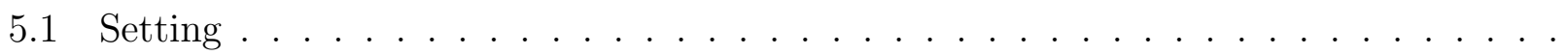

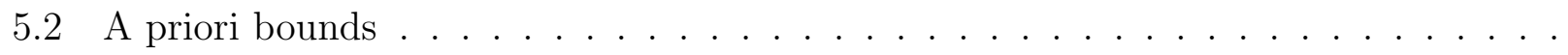

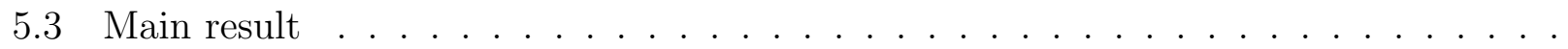

\section{Examples}

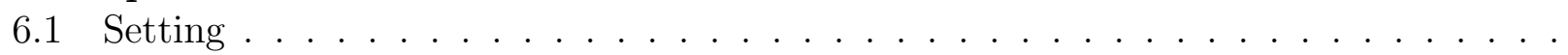

6.2 Auxiliary lemmas . . . . . . . . . . . . . . . . . . . . . .

6.3 Strong convergence rates for a nonlinearity-truncated exponential Euler scheme . . .

6.4 Strong convergence rates for a nonlinearity-truncated linear-implicit Euler scheme .

\section{Introduction}

In this article we study strong numerical approximations of semilinear stochastic evolution equations (SEEs) with superlinearly growing nonlinearities. The explicit Euler scheme and the linear-implicit Euler scheme are known to diverge strongly and numerically weakly in the case of such SEEs; see Theorem 2.1 in Hutzenthaler et al. [19], Theorem 2.1 in Hutzenthaler al. [21], and Section 5.1 in Kurniawan [29]. Fully drift-implicit Euler schemes have been shown to converge strongly even in the case of some SEEs with superlinearly growing nonlinearities and thereby overcome the lack of strong convergence of the explicit and the linear-implicit Euler scheme; see, e.g., Theorem 2.4 in Hu [16], Theorem 2.10 Gyöngy \& Millet [11], and Theorem 1.1 in Kovács et al. [26]. Fully drift-implicit Euler schemes can, however, often only be simulated approximatively as a nonlinear equation has to be solved in each time step and the resulting approximations of the fully drift-implicit Euler approximations require additional computational effort (particularly, when the state space of the considered SEE is high dimensional, see, e.g., Figure 4 in Hutzenthaler et al. [20]) and have not yet been shown to converge strongly. Recently, a series of explicit and easily implementable timediscrete approximation schemes have been proposed and shown to converge strongly in the case of SEEs with superlinearly growing nonlinearities; see, e.g., Hutzenthaler et al. [20], Wang \& Gan [45], Hutzenthaler \& Jentzen [18], Tretyakov \& Zhang [44], Halidias [13], Sabanis [39, 40], Halidias \& Stamatiou [15], Hutzenthaler et al. [22], Szpruch \& Zhāng [42], Halidias [14], Liu \& Mao [30], Hutzenthaler \& Jentzen [17], Zhang [46], Dareiotis et al. [10], Kumar \& Sabanis [27], Beyn et al. [1], Zong et al. [47], Song et al. [41], Ngo \& Luong [36], Tambue \& Mukam [43], Mao [34], Beyn et al. [2], Kumar \& Sabanis [28], and Mao [35] in the case of finite dimensional SEEs and see, e.g., Gyöngy et al. [12] and Jentzen \& Pušnik [25] in the case of infinte dimensional SEEs. These schemes are suitable modified versions of the explicit Euler scheme that somehow tame/truncate the superlinearly growing nonlinearities of the considered SEE and thereby prevent the considered tamed 
scheme from strong divergence. However, each of the above mentioned temporal strong convergence results for implicit (see [16, 11, 26]) and explicit (see [20, 45, 18, 44, 13, 39, 40, 15, 22, 42, 14, 30, 17, 46, 10, 27, 12, 1, 47, 41, 25, 36, 43, 34, 2, 28, 35]) schemes applies merely to trace noise class driven SEEs and excludes the important case of the more irregular space-time white noise. In particular, none of these results applies to space-time white noise driven stochastic Ginzburg-Landau equations. In this work we intend to close this gap and we propose (see (3) and (4) below) and analyze (see Theorem 5.3 below) suitable explicit and strongly convergent approximation schemes for possibly space-time white noise driven SEEs. In particular, we establish essentially sharp strong convergence rates for suitable explicit nonlinearity-truncated approximation schemes for space-time white noise driven stochastic Ginzburg-Landau equations (see (5), Corollary 6.15, and Corollary 6.17 below).

To illustrate the main result of this article (see Theorem 5.3 in Section 5.3 below) in more detail, we consider the following example of our general setting (see Section 5.11) in this introductory section. Let $T \in(0, \infty), n \in\{1,3,5, \ldots\}, a_{0}, a_{1}, \ldots, a_{n-1} \in \mathbb{R}, a_{n} \in(-\infty, 0), \xi \in H_{0}^{2}((0,1) ; \mathbb{R})$, $H=L^{2}((0,1) ; \mathbb{R})$, let $F: L^{2 n}((0,1) ; \mathbb{R}) \rightarrow H$ be the function with the property that for all $v \in$ $L^{2 n}((0,1) ; \mathbb{R})$ it holds that $F(v)=\sum_{k=0}^{n} a_{k} v^{k}$, let $A: D(A) \subseteq H \rightarrow H$ be the Laplacian with Dirichlet boundary conditions on $H$, let $(\Omega, \mathcal{F}, \mathbb{P})$ be a probability space with a normal filtration $\left(\mathcal{F}_{t}\right)_{t \in[0, T]}$, and let $\left(W_{t}\right)_{t \in[0, T]}$ be an $\operatorname{Id}_{H^{-c y l i n d r i c a l ~}}\left(\mathcal{F}_{t}\right)_{t \in[0, T]}$-Wiener process. The assumptions that $n$ is odd and that $a_{n}<0$ ensure that there exists an up to indistinguishability unique $\left(\mathcal{F}_{t}\right)_{t \in[0, T]^{-}}$ adapted stochastic process $X:[0, T] \times \Omega \rightarrow L^{2 n}((0,1) ; \mathbb{R})$ with continuous sample paths which satisfies that for all $t \in[0, T]$ it holds $\mathbb{P}$-a.s. that

$$
X_{t}=e^{t A} \xi+\int_{0}^{t} e^{(t-s) A} F\left(X_{s}\right) d s+\int_{0}^{t} e^{(t-s) A} d W_{s}
$$

(cf., e.g., Section 7.2 in Da Prato \& Zabczyk [8] and Chapter 6 in Cerrai [4]). The stochastic process $X$ is thus a mild solution of the SPDE

$$
d X_{t}(x)=\left[\frac{\partial^{2}}{\partial x^{2}} X_{t}(x)+\sum_{k=0}^{n} a_{k}\left(X_{t}(x)\right)^{k}\right] d t+d W_{t}(x)
$$

with $X_{0}(x)=\xi(x)$ and $X_{t}(0)=X_{t}(1)=0$ for $x \in(0,1), t \in[0, T]$. In the case $n=3$ and $a_{0}=a_{2}=0$, the SPDE (2) is often referred to as stochastic Ginzburg-Landau equation in the literature (see also (6) below). In this article we introduce the following nonlinearity-truncated exponential scheme to approximate the solution process $X$ of the SPDE (2) . Let $\lfloor\cdot\rfloor_{h}: \mathbb{R} \rightarrow \mathbb{R}$, $h \in(0, \infty)$, be the mappings with the property that for all $h \in(0, \infty), t \in \mathbb{R}$ it holds that $\lfloor t\rfloor_{h}=$ $\max (\{0, h,-h, 2 h,-2 h, \ldots\} \cap(-\infty, t])$, let $\chi \in\left(0, \frac{1}{2 n}\right]$, and let $Y^{N}:[0, T] \times \Omega \rightarrow L^{2 n^{2}}((0,1) ; \mathbb{R})$, $N \in \mathbb{N}$, be stochastic processes which satisfy that for all $t \in[0, T], N \in \mathbb{N}$ it holds $\mathbb{P}$-a.s. that

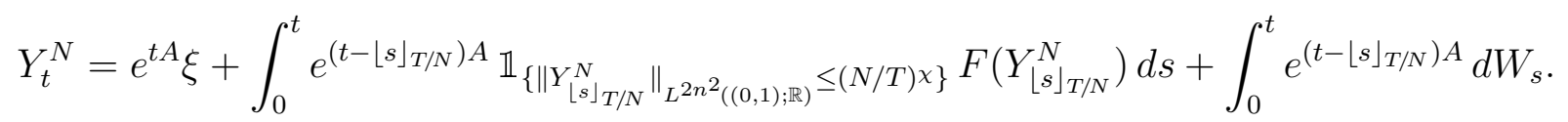

The approximation scheme (3) is a nonlinearity-truncated modification (cf. (2) in Jentzen \& Pušnik [25]) of a time-continuous version (cf., e.g., (130)-(134) in Da Prato et al. [7]) of the classical exponential Euler approximation scheme for semilinear SPDEs (cf., e.g., (3.2) and (3.6) in Lord \& Rougemont [31]). We also propose the following nonlinearity-truncated linear-implicit scheme to approximate the solution process $X$ of the $\operatorname{SPDE}(2)$. Let $Z^{N}:[0, T] \times \Omega \rightarrow L^{2 n^{2}}((0,1) ; \mathbb{R}), N \in \mathbb{N}$, 
be stochastic processes which satisfy that for all $t \in[0, T], N \in \mathbb{N}$ it holds $\mathbb{P}$-a.s. that

$$
\begin{aligned}
& Z_{t}^{N}=\left(\operatorname{Id}_{H}-\left(t-\lfloor t\rfloor_{T / N}\right) A\right)^{-1}\left(\operatorname{Id}_{H}-\frac{T}{N} A\right)^{-\lfloor t\rfloor_{T / N} \frac{N}{T}} \xi \\
& +\int_{0}^{t}\left(\operatorname{Id}_{H}-\left(t-\lfloor t\rfloor_{T / N}\right) A\right)^{-1}\left(\operatorname{Id}_{H}-\frac{T}{N} A\right)^{\left(\lfloor s\rfloor_{T / N}-\lfloor t\rfloor_{T / N}\right) \frac{N}{T}} \mathbb{1}_{\left\{\left\|Z_{\lfloor s\rfloor_{T / N}^{N}}\right\|_{L^{2 n^{2}((0,1) ; \mathbb{R})}} \leq(N / T)^{\chi}\right\}} F\left(Z_{\lfloor s\rfloor_{T / N}}^{N}\right) d s \\
& +\int_{0}^{t}\left(\operatorname{Id}_{H}-\left(t-\lfloor t\rfloor_{T / N}\right) A\right)^{-1}\left(\operatorname{Id}_{H}-\frac{T}{N} A\right)^{\left(\lfloor s\rfloor_{T / N}-\lfloor t\rfloor_{T / N}\right) \frac{N}{T}} d W_{s} .
\end{aligned}
$$

The approximation scheme (44) is a nonlinearity-truncated modification (cf. (2) in Jentzen \& Pušnik [25]) of a time-continuous version (cf., e.g., (142)-(146) in Da Prato et al. [7]) of the classical linear-implicit Euler approximation scheme for semilinear SPDEs. Both approximation schemes, (3) and (4), are easy to implement (cf. (7) and (8) below and cf., e.g., also Section 4 in Lord \& Tambue [32]). In Corollary 6.15 and Corollary 6.17below we prove that for all $p \in(0, \infty), \theta \in[0,1 / 4)$ there exists a real number $C \in \mathbb{R}$ such that for all $N \in \mathbb{N}$ it holds that

$$
\sup _{t \in[0, T]}\left(\mathbb{E}\left[\left\|X_{t}-Y_{t}^{N}\right\|_{H}^{p}\right]\right)^{1 / p}+\sup _{t \in[0, T]}\left(\mathbb{E}\left[\left\|X_{t}-Z_{t}^{N}\right\|_{H}^{p}\right]\right)^{1 / p} \leq C N^{-\theta} .
$$

Our proofs of (5), Corollary 6.15, and Corollary 6.17, respectively, are based on the well known idea to substract the Ornstein-Uhlenbeck process from the solution process of the SPDE (cf. Section 2 below and, e.g., (14.2.2)-(14.2.3) in Section 14.2 in Da Prato \& Zabczyk [9]), are based on some arguments in Jentzen \& Kurniawan [24] and Jentzen \& Pušnik [25], and are based on an appropriate Lyapunov-type condition for one-step approximation schemes on Banach spaces (see (10) in Section 2.1, (85) in Section 5.1, and Corollary 6.3). To the best of our knowledge, inequality (5), Corollary 6.15, and Corollary 6.17, respectively, are the first results in the literature that establish strong convergence for temporal numerical approximations of the SPDE (2) in the case where $n>1$.

In the following we illustrate (5) through a numerical example. For this we consider the choice $T=1, n=3, a_{0}=0, a_{1}=1, a_{2}=0, a_{3}=-1, \xi=0, \chi=1 / 6=1 / 2 n$ and we note that the SPDE (2) reduces in this case to the stochastic Ginzburg-Landau equation

$$
d X_{t}(x)=\left[\frac{\partial^{2}}{\partial x^{2}} X_{t}(x)+X_{t}(x)-\left(X_{t}(x)\right)^{3}\right] d t+d W_{t}(x)
$$

with $X_{0}(x)=0$ and $X_{t}(0)=X_{t}(1)=0$ for $x \in(0,1), t \in[0, T]$. Note that the approximation processes in (3) and (44) satisfy in the case of (6) that for all $n \in\{1,2, \ldots, N-1\}, N \in \mathbb{N}$ it holds $\mathbb{P}$-a.s. that $Y_{0}^{N}=Z_{0}^{N}=0$ and

$$
\begin{aligned}
& Y_{(n+1) / N}^{N}=e^{\frac{T}{N} A}\left[Y_{n / N}^{N}+\frac{T}{N} \mathbb{1}_{\left\{\left\|Y_{n / N}^{N}\right\|_{L^{18}((0,1) ; \mathbb{R})} \leq N^{1 / 6}\right\}}\left(Y_{n / N}^{N}-\left[Y_{n / N}^{N}\right]^{3}\right)+\int_{n / N}^{(n+1) / N} d W_{s}\right], \\
& Z_{(n+1) / N}^{N}=\left(\operatorname{Id}_{H}-\frac{T}{N} A\right)^{-1}\left[Z_{n / N}^{N}+\frac{T}{N} \mathbb{1}_{\left\{\left\|Z_{n / N}^{N}\right\|_{L 18((0,1) ; \mathbb{R})} \leq N^{1 / 6}\right\}}\left(Z_{n / N}^{N}-\left[Z_{n / N}^{N}\right]^{3}\right)+\int_{n / N}^{(n+1) / N} d W_{s}\right] .
\end{aligned}
$$

In Figure 1 we plot approximatively the strong root mean square approximation errors

$$
\left(\mathbb{E}\left[\left\|X_{T}-Y_{T}^{N}\right\|_{H}^{2}\right]\right)^{1 / 2} \quad \text { and } \quad\left(\mathbb{E}\left[\left\|X_{T}-Z_{T}^{N}\right\|_{H}^{2}\right]\right)^{1 / 2}
$$


against the number of time steps $N \in\{64,128,256, \ldots, 262144\}$. The infinite dimensional Hilbert space $H=L^{2}((0,1) ; \mathbb{R})$ in $(9)$ is approximated in Figure 1 through the finite dimensional subspace spanned by the first 1024 eigenfunctions of the Laplacian, the unknown exact solution process $\left(X_{t}\right)_{t \in[0, T]}$ of (6) is approximated in Figure 1 through a nonlinearity-truncated linear-implicit CrankNicolson approximation with 1048576 time steps, and the expectations in (9) are approximated in Figure 1 by a Monte Carlo approximation with 25 Monte Carlo runs. Details can be found in Figure 2 in which we present the MATLAB code that has been used to create Figure 1. The order lines in Figure 1 correspond to the convergence orders 1/8, 1/4, and 1/2. Figure 1 thus essentially agrees with our strong convergence result (5) which proves that both schemes, (77) and (8), converge in the sense of (9) with order $1 / 4-$.

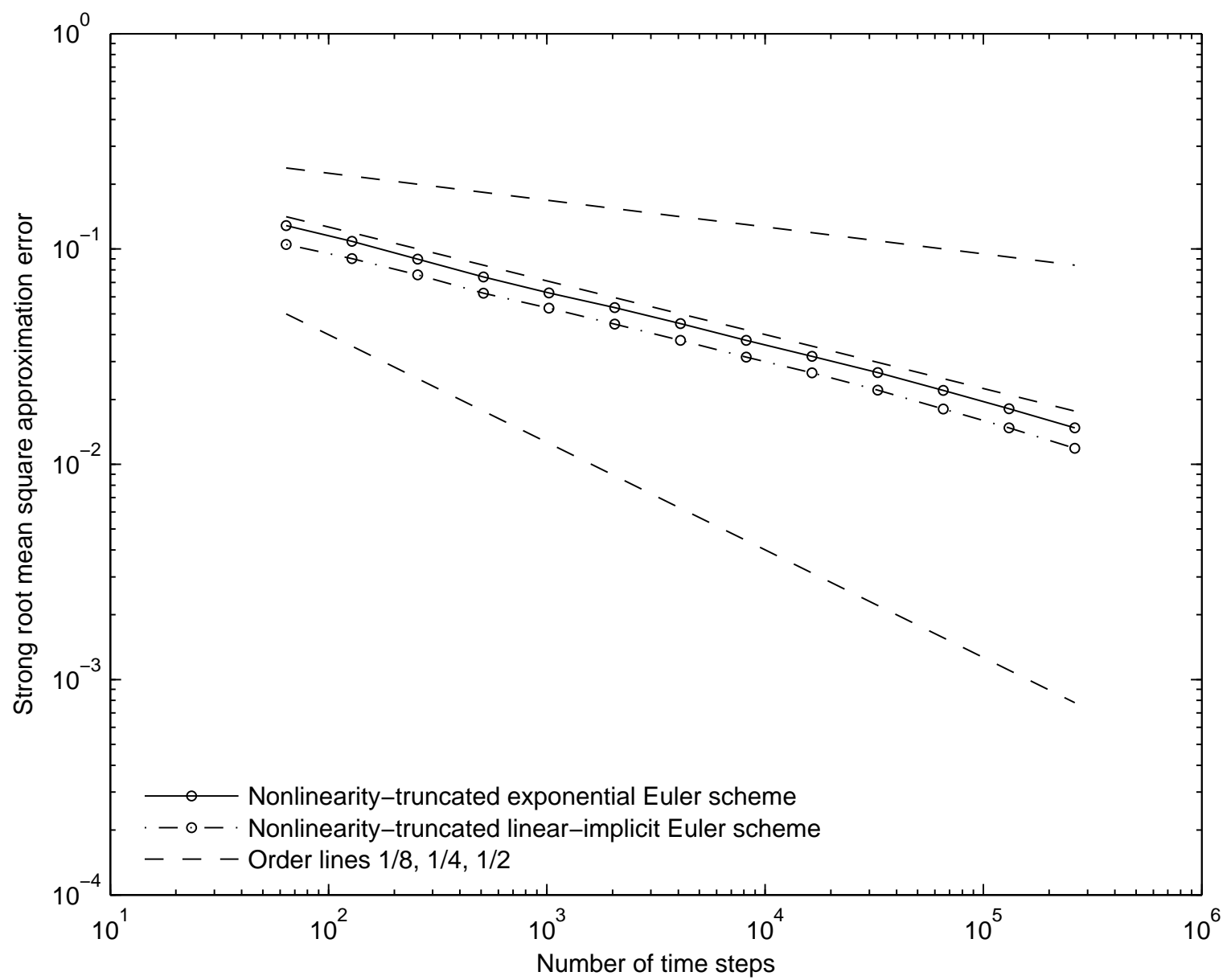

Figure 1: Strong approximation errors (9) against the number of time steps $N \in\{64,128$, $256, \ldots, 262144\}$.

The remainder of this article is organized as follows. In Section 2 the required a priori moments for the nonlinearity-truncated approximation schemes are established, in Section 3 the error analysis is performed in the pathwise sense under the hypothesis of suitable a priori bounds for the approximation processes, and in Section 4 the error analysis is carried out in the strong $L^{p}$-sense 


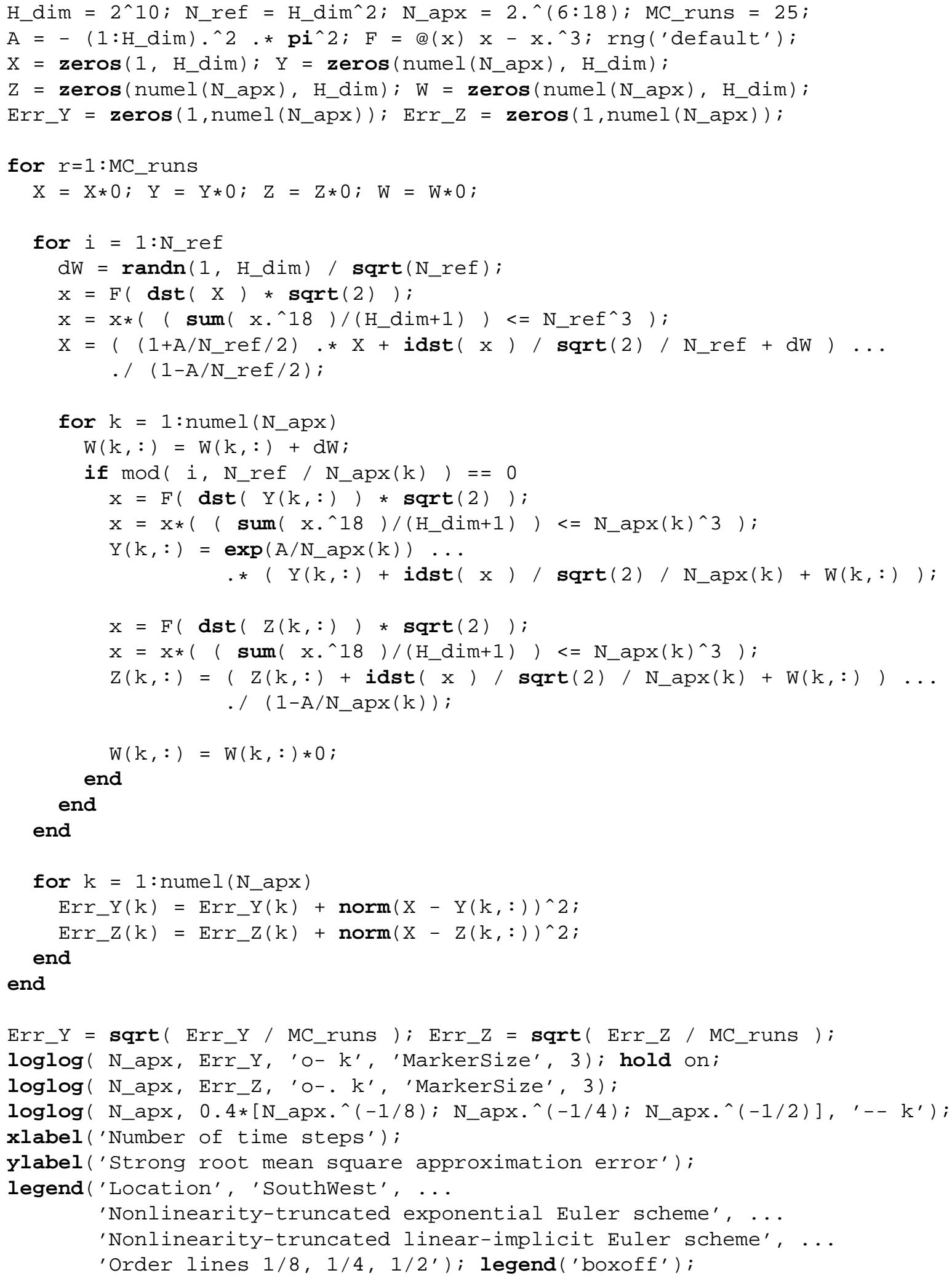

Figure 2: MATLAB code used to create Figure 1. 
under the hypothesis of appropriate a priori moment bounds for the approximation processes. Section 5 combines the results of Section 2 and Section 4 and thereby establishes Theorem 5.3 which is the main result of this article. The analysis in Sections 25 is carried out for abstract stochastic evolution equations on separable Banach and Hilbert spaces, respectively. Section 6 then verifies that the assumptions of Theorem 5.3 in Section 5 are satisfied in the case of concrete stochastic partial differential equations of the type (2) and, in particular, establishes Corollary 6.15] and Corollary 6.17.

\subsection{Notation}

Throughout this article the following notation is used. For measurable spaces $\left(\Omega_{1}, \mathcal{F}_{1}\right)$ and $\left(\Omega_{2}, \mathcal{F}_{2}\right)$ we denote by $\mathcal{M}\left(\mathcal{F}_{1}, \mathcal{F}_{2}\right)$ the set of all $\mathcal{F}_{1} / \mathcal{F}_{2}$-measurable mappings. For a set $A$ we denote by $\mathcal{P}(A)$ the power set of $A$. For a set $A$ and a subset $\mathcal{A} \subseteq \mathcal{P}(A)$ we denote by $\sigma_{A}(\mathcal{A})$ the smallest sigma-algebra on $A$ which contains $\mathcal{A}$. For a topological space $(X, \tau)$ we denote by $\mathcal{B}(X)$ the set given by $\mathcal{B}(X)=\sigma_{X}(\tau)$. For a natural number $d \in \mathbb{N}$ and a set $A \in \mathcal{B}\left(\mathbb{R}^{d}\right)$ we denote by $\lambda_{A}: \mathcal{B}(A) \rightarrow[0, \infty]$ the Lebesgue-Borel measure on $A$. We denote by $\left\lfloor_{h} \cdot\right\rfloor_{h}: \mathbb{R} \rightarrow \mathbb{R}, h \in(0, \infty)$, and $\lceil\cdot\rceil_{h}: \mathbb{R} \rightarrow \mathbb{R}, h \in(0, \infty)$, the mappings with the property that for all $h \in(0, \infty), t \in \mathbb{R}$ it holds that $\lfloor t\rfloor_{h}=\max (\{0, h,-h, 2 h,-2 h, \ldots\} \cap(-\infty, t])$ and $\lceil t\rceil_{h}=\min (\{0, h,-h, 2 h,-2 h, \ldots\} \cap[t, \infty))$. For a measure space $(\Omega, \mathcal{F}, \mu)$, a measurable space $(S, \mathcal{S})$, a set $R \subseteq S$, and a function $f: \Omega \rightarrow R$ we denote by $[f]_{\mu, \mathcal{S}}$ the set given by $[f]_{\mu, \mathcal{S}}=\{g \in \mathcal{M}(\mathcal{F}, \mathcal{S}):(\exists A \in \mathcal{F}: \mu(A)=0$ and $\{\omega \in \Omega: f(\omega) \neq$ $g(\omega)\} \subseteq A)\}$.

\section{$2 \quad$ A priori bounds}

\subsection{Setting}

Let $T, \phi \in(0, \infty), c, C \in[0, \infty), M \in \mathbb{N}$, let $\left(V,\|\cdot\|_{V}\right)$ and $\left(W,\|\cdot\|_{W}\right)$ be separable $\mathbb{R}$-Banach spaces with $V \subseteq W$ densely and continuously, let $\Phi, \Psi, \mathcal{V} \in \mathcal{M}(\mathcal{B}(V), \mathcal{B}([0, \infty))), F \in \mathcal{M}(\mathcal{B}(V), \mathcal{B}(W))$, $\mathcal{S} \in \mathcal{M}\left(\mathcal{B}\left(\left\{(s, t) \in[0, T]^{2}: s<t\right\}\right), \mathcal{B}(L(W, V))\right)$ satisfy that for all $u, v \in V, r_{1}, r_{2}, r_{3} \in[0, T]$, $s \in\left\{0, \frac{T}{M}, \frac{2 T}{M}, \ldots, \frac{(M-1) T}{M}\right\}, t \in\left(s, s+\frac{T}{M}\right]$ with $r_{1}<r_{2}<r_{3}$ it holds that $\|F(u+v)\|_{W} \leq C(1+$ $\left.|\Phi(u)|^{\phi}+|\Phi(v)|^{\phi}\right), \mathcal{S}_{r_{1}, r_{3}}=\mathcal{S}_{r_{2}, r_{3}} \mathcal{S}_{r_{1}, r_{2}}$, and

$$
\Phi\left(\mathcal{S}_{s, t}\left[u+(t-s) \mathbb{1}_{[0, M / T]}(\mathcal{V}(u+v)) F(u+v)\right]\right) \leq e^{c(t-s)}[\Phi(u)+(t-s) \Psi(v)],
$$

let $(\Omega, \mathcal{F}, \mathbb{P})$ be a probability space, and let $O, Y:[0, T] \times \Omega \rightarrow V$ be stochastic processes such that for all $t \in(0, T]$ it holds that

$$
Y_{t}=\mathcal{S}_{0, t} Y_{0}+\int_{0}^{t} \mathcal{S}_{\lfloor s\rfloor_{T / M}, t} \mathbb{1}_{\left\{\mathcal{V}\left(Y_{\lfloor s\rfloor_{T / M}}+O_{\lfloor s\rfloor_{T / M}}\right) \leq M / T\right\}} F\left(Y_{\lfloor s\rfloor_{T / M}}+O_{\lfloor s\rfloor_{T / M}}\right) d s .
$$

\subsection{A priori bounds based on variational arguments}

Lemma 2.1. Assume the setting in Section 2.1. Then for all $t \in[0, T]$ it holds that

$$
\Phi\left(Y_{t}\right) \leq e^{c t} \Phi\left(Y_{0}\right)+\int_{0}^{t} e^{c\left(t-\lfloor s\rfloor_{T / M}\right)} \Psi\left(O_{\lfloor s\rfloor_{T / M}}\right) d s
$$


Proof of Lemma 2.1. Note that (11) shows that for all $s \in\left\{0, \frac{T}{M}, \frac{2 T}{M}, \ldots, \frac{(M-1) T}{M}\right\}, t \in\left(s, s+\frac{T}{M}\right]$ it holds that

$$
Y_{t}=\mathcal{S}_{s, t}\left[Y_{s}+(t-s) \mathbb{1}_{\left\{\mathcal{V}\left(Y_{s}+O_{s}\right) \leq M / T\right\}} F\left(Y_{s}+O_{s}\right)\right]
$$

This and (10) prove that for all $s \in\left\{0, \frac{T}{M}, \frac{2 T}{M}, \ldots, \frac{(M-1) T}{M}\right\}, t \in\left(s, s+\frac{T}{M}\right]$ it holds that

$$
\Phi\left(Y_{t}\right) \leq e^{c(t-s)}\left[\Phi\left(Y_{s}\right)+(t-s) \Psi\left(O_{s}\right)\right] .
$$

In particular, (14) implies that for all $m \in\{1,2, \ldots, M\}$ it holds that

$$
\begin{aligned}
\Phi\left(Y_{\frac{m T}{M}}\right) & \leq e^{c \frac{T}{M}}\left[\Phi\left(Y_{\frac{(m-1) T}{M}}\right)+\frac{T}{M} \Psi\left(O_{\frac{(m-1) T}{M}}\right)\right] \leq \ldots \leq e^{c \frac{m T}{M}} \Phi\left(Y_{0}\right)+\frac{T}{M} \sum_{l=0}^{m-1} e^{c(m-l) \frac{T}{M}} \Psi\left(O_{\frac{l T}{M}}\right) \\
& =e^{c \frac{m T}{M}} \Phi\left(Y_{0}\right)+\sum_{l=0}^{m-1} \int_{\frac{l T}{M}}^{\frac{(l+1) T}{M}} e^{c\left(\frac{m T}{M}-\lfloor s\rfloor_{T / M}\right)} \Psi\left(O_{\lfloor s\rfloor_{T / M}}\right) d s \\
& =e^{c \frac{m T}{M}} \Phi\left(Y_{0}\right)+\int_{0}^{\frac{m T}{M}} e^{c\left(\frac{m T}{M}-\lfloor s\rfloor_{T / M}\right)} \Psi\left(O_{\lfloor s\rfloor_{T / M}}\right) d s .
\end{aligned}
$$

Moreover, (14) and (15) yield that for all $t \in[0, T]$ it holds that

$$
\begin{aligned}
& \Phi\left(Y_{t}\right) \\
& \leq e^{c\left(t-\lfloor t\rfloor_{T / M}\right)}\left[\Phi\left(Y_{\lfloor t\rfloor_{T / M}}\right)+\left(t-\lfloor t\rfloor_{T / M}\right) \Psi\left(O_{\lfloor t\rfloor_{T / M}}\right)\right] \\
& \leq e^{c\left(t-\lfloor t\rfloor_{T / M}\right)}\left[e^{c\lfloor t\rfloor_{T / M}} \Phi\left(Y_{0}\right)+\int_{0}^{\lfloor t\rfloor_{T / M}} e^{c\left(\lfloor t\rfloor_{T / M}-\lfloor s\rfloor_{T / M}\right)} \Psi\left(O_{\lfloor s\rfloor_{T / M}}\right) d s+\left(t-\lfloor t\rfloor_{T / M}\right) \Psi\left(O_{\lfloor t\rfloor_{T / M}}\right)\right] \\
& =e^{c t} \Phi\left(Y_{0}\right)+\int_{0}^{t} e^{c\left(t-\lfloor s\rfloor_{T / M}\right)} \Psi\left(O_{\lfloor s\rfloor_{T / M}}\right) d s .
\end{aligned}
$$

This completes the proof of Lemma 2.1.

\subsection{A priori bounds based on bootstrap-type arguments}

Lemma 2.2. Assume the setting in Section 2.1 and let $t \in[0, T]$. Then

$$
\left\|F\left(Y_{t}+O_{t}\right)\right\|_{W} \leq C\left(1+2^{[\phi-1]^{+}} e^{c \phi t}\left[\left|\Phi\left(Y_{0}\right)\right|^{\phi}+\left|\int_{0}^{t} \Psi\left(O_{\lfloor s\rfloor_{T / M}}\right) d s\right|^{\phi}\right]+\left|\Phi\left(O_{t}\right)\right|^{\phi}\right) .
$$

Proof of Lemma 2.2. Note that the assumption that $\forall u, v \in V:\|F(u+v)\|_{W} \leq C\left(1+|\Phi(u)|^{\phi}+\right.$ $\left.|\Phi(v)|^{\phi}\right)$, Lemma 2.1, and the fact that $\forall x, y \in \mathbb{R}, r \in(0, \infty):|x+y|^{r} \leq 2^{[r-1]^{+}}|x|^{r}+2^{[r-1]^{+}}|y|^{r}$ 
imply that

$$
\begin{aligned}
\left\|F\left(Y_{t}+O_{t}\right)\right\|_{W} & \leq C\left(1+\left|\Phi\left(Y_{t}\right)\right|^{\phi}+\left|\Phi\left(O_{t}\right)\right|^{\phi}\right) \\
& \leq C\left(1+\left|e^{c t} \Phi\left(Y_{0}\right)+\int_{0}^{t} e^{c\left(t-\lfloor s\rfloor_{T / M}\right)} \Psi\left(O_{\lfloor s\rfloor_{T / M}}\right) d s\right|^{\phi}+\left|\Phi\left(O_{t}\right)\right|^{\phi}\right) \\
& \leq C\left(1+2^{[\phi-1]^{+}}\left|e^{c t} \Phi\left(Y_{0}\right)\right|^{\phi}+2^{[\phi-1]^{+}}\left|\int_{0}^{t} e^{c\left(t-\lfloor s\rfloor_{T / M}\right)} \Psi\left(O_{\lfloor s\rfloor_{T / M}}\right) d s\right|^{\phi}+\left|\Phi\left(O_{t}\right)\right|^{\phi}\right) .
\end{aligned}
$$

This completes the proof of Lemma 2.2 .

Corollary 2.3. Assume the setting in Section 2.1 and let $t \in(0, T], \rho \in[0,1)$. Then

$$
\begin{aligned}
\left\|Y_{t}\right\|_{V} \leq & \left\|\mathcal{S}_{0, t}\right\|_{L(V)}\left\|Y_{0}\right\|_{V}+C\left[\sup _{s \in[0, t)}(t-s)^{\rho}\left\|\mathcal{S}_{\lfloor s\rfloor_{T / M}, t}\right\|_{L(W, V)}\right]\left[\int_{0}^{t}(t-s)^{-\rho}\left|\Phi\left(O_{\lfloor s\rfloor_{T / M}}\right)\right|^{\phi} d s\right. \\
& \left.+\frac{t^{(1-\rho)}}{(1-\rho)}\left(1+2^{[\phi-1]^{+}} e^{c \phi t}\left[\left|\Phi\left(Y_{0}\right)\right|^{\phi}+\left|\int_{0}^{t} \Psi\left(O_{\lfloor u\rfloor_{T / M}}\right) d u\right|^{\phi}\right]\right)\right] .
\end{aligned}
$$

Proof of Corollary 2.3. Note that Lemma 2.2 implies

$$
\begin{aligned}
\left\|Y_{t}\right\|_{V} \leq & \left\|\mathcal{S}_{0, t} Y_{0}\right\|_{V}+\int_{0}^{t}\left\|\mathcal{S}_{\lfloor s\rfloor_{T / M}, t}\right\|_{L(W, V)}\left\|\mathbb{1}_{\left\{\mathcal{V}\left(Y_{\lfloor s\rfloor_{T / M}}+O_{\lfloor s\rfloor}\right) \leq M / T\right\}} F\left(Y_{\lfloor s\rfloor_{T / M}}+O_{\lfloor s\rfloor_{T / M}}\right)\right\|_{W} d s \\
\leq & \left\|\mathcal{S}_{0, t}\right\|_{L(V)}\left\|Y_{0}\right\|_{V} \\
& +\left[\sup _{s \in[0, t)}\left(t-\lfloor s\rfloor_{T / M}\right)^{\rho}\left\|\mathcal{S}_{\lfloor s\rfloor_{T / M}, t}\right\|_{L(W, V)}\right] \int_{0}^{t}\left(t-\lfloor s\rfloor_{T / M}\right)^{-\rho}\left\|F\left(Y_{\lfloor s\rfloor_{T / M}}+O_{\lfloor s\rfloor_{T / M}}\right)\right\|_{W} d s \\
\leq & \left\|\mathcal{S}_{0, t}\right\|_{L(V)}\left\|Y_{0}\right\|_{V}+C\left[\sup _{s \in[0, t)}(t-s)^{\rho}\left\|\mathcal{S}_{\lfloor s\rfloor_{T / M}, t}\right\|_{L(W, V)}\right]\left[\int _ { 0 } ^ { t } ( t - s ) ^ { - \rho } \left(1+\left|\Phi\left(O_{\lfloor s\rfloor_{T / M}}\right)\right|^{\phi}\right.\right. \\
& \left.\left.+2^{[\phi-1]^{+}} e^{c \phi\lfloor s\rfloor_{T / M}}\left[\left|\Phi\left(Y_{0}\right)\right|^{\phi}+\left|\int_{0}^{\lfloor s\rfloor_{T / M}} \Psi\left(O_{\lfloor u\rfloor_{T / M}}\right) d u\right|^{\phi}\right]\right) d s\right] .
\end{aligned}
$$

This completes the proof of Corollary 2.3.

Corollary 2.4. Assume the setting in Section 2.1 and let $p \in[\max \{1,1 / \phi\}, \infty), t \in(0, T], \rho \in[0,1)$. Then

$$
\begin{aligned}
& \left\|Y_{t}\right\|_{\mathcal{L}^{p}(\mathbb{P} ; V)} \leq\left\|\mathcal{S}_{0, t}\right\|_{L(V)}\left\|Y_{0}\right\|_{\mathcal{L}^{p}(\mathbb{P} ; V)}+\frac{C t^{(1-\rho)}}{(1-\rho)}\left[\sup _{s \in[0, t)}(t-s)^{\rho}\left\|\mathcal{S}_{\lfloor s\rfloor_{T / M}, t}\right\|_{L(W, V)}\right] \\
& \cdot\left(1+\sup _{s \in[0, t)}\left\|\Phi\left(O_{s}\right)\right\|_{\mathcal{L}^{p \phi(\mathbb{P} ; \mathbb{R})}}^{\phi}+2^{[\phi-1]^{+}} e^{c \phi t}\left[\left\|\Phi\left(Y_{0}\right)\right\|_{\mathcal{L}^{p \phi(\mathbb{P} ; \mathbb{R})}}^{\phi}+t^{\phi} \sup _{s \in[0, t)}\left\|\Psi\left(O_{s}\right)\right\|_{\mathcal{L}^{p \phi(\mathbb{P} ; \mathbb{R})}}^{\phi}\right]\right) .
\end{aligned}
$$


Proof of Corollary 2.4. Observe that Corollary 2.3 implies

$$
\begin{aligned}
& \left\|Y_{t}\right\|_{\mathcal{L}^{p}(\mathbb{P} ; V)} \leq\left\|\mathcal{S}_{0, t}\right\|_{L(V)}\left\|Y_{0}\right\|_{\mathcal{L}^{p}(\mathbb{P} ; V)} \\
& +C\left[\sup _{s \in[0, t)}(t-s)^{\rho}\left\|\mathcal{S}_{\lfloor s\rfloor_{T / M}, t}\right\|_{L(W, V)}\right]\left[\int_{0}^{t}(t-s)^{-\rho}\left\|\Phi\left(O_{\lfloor s\rfloor_{T / M}}\right)\right\|_{\mathcal{L}^{p \phi}(\mathbb{P} ; \mathbb{R})}^{\phi} d s\right. \\
& \left.+\frac{t^{(1-\rho)}}{(1-\rho)}\left(1+2^{[\phi-1]^{+}} e^{c \phi t}\left[\left\|\Phi\left(Y_{0}\right)\right\|_{\mathcal{L}^{p \phi}(\mathbb{P} ; \mathbb{R})}^{\phi}+\left|\int_{0}^{t}\left\|\Psi\left(O_{\lfloor u\rfloor_{T / M}}\right)\right\|_{\mathcal{L}^{p \phi}(\mathbb{P} ; \mathbb{R})} d u\right|^{\phi}\right]\right)\right] .
\end{aligned}
$$

The proof of Corollary 2.4 is thus completed.

\section{Pathwise error estimates}

\subsection{Setting}

Let $T, C, \varphi \in(0, \infty), L \in[0, \infty), \rho \in[0,1), M \in \mathbb{N}$, let $\left(H,\langle\cdot, \cdot\rangle_{H},\|\cdot\|_{H}\right)$ be a separable $\mathbb{R}$-Hilbert space, let $A: D(A) \subseteq H \rightarrow H$ be a generator of an analytic semigroup with $\operatorname{spectrum}(A) \subseteq\{z \in$ $\mathbb{C}: \operatorname{Re}(z)<0\}$, let $\left(H_{r},\langle\cdot, \cdot\rangle_{H_{r}},\|\cdot\|_{H_{r}}\right), r \in \mathbb{R}$, be a family of interpolation spaces associated to $-A$ (cf., e.g., Definition 3.5.26 in [23]), let $\left(V,\|\cdot\|_{V}\right)$ be a separable $\mathbb{R}$-Banach space with $H_{1} \subseteq$ $V \subseteq H$ densely and continuously, let $\mathcal{V} \in \mathcal{M}(\mathcal{B}(V), \mathcal{B}([0, \infty))), \mathcal{S} \in \mathcal{M}\left(\mathcal{B}\left(\left\{(s, t) \in[0, T]^{2}: s<\right.\right.\right.$ $t\}), \mathcal{B}(L(H, V))), F \in \mathcal{C}(V, H)$ satisfy for all $x, y \in V$ with $x-y \in H_{1}$ that $\|F(x)-F(y)\|_{H}^{2} \leq$ $L\|x-y\|_{V}^{2}\left(1+\|x\|_{V}^{\varphi}+\|y\|_{V}^{\varphi}\right)$ and $\langle x-y, A(x-y)+F(x)-F(y)\rangle_{H} \leq C\|x-y\|_{H}^{2}$, assume that $\sup _{t \in(0, T)} t^{\rho}\left\|e^{t A}\right\|_{L(H, V)}<\infty$, let $(\Omega, \mathcal{F}, \mathbb{P})$ be a probability space, let $X:[0, T] \times \Omega \rightarrow V$ be a stochastic process with continuous sample paths, let $O:[0, T] \times \Omega \rightarrow V$ be a stochastic process which satisfies for all $\omega \in \Omega$ that $\limsup _{r \searrow 0} \sup _{0 \leq s<t \leq T} \frac{s\left\|O_{t}(\omega)-O_{s}(\omega)\right\|_{V}}{(t-s)^{r}}<\infty$, let $\mathcal{O}, \bar{X}, \mathcal{X}, \overline{\mathcal{X}}, \mathbf{X}:[0, T] \times$ $\Omega \rightarrow V$ be stochastic processes, and assume for all $t \in[0, T]$ that $X_{t}=\int_{0}^{t} e^{(t-s) A} F\left(X_{s}\right) d s+O_{t}$, $\bar{X}_{t}=X_{t}-O_{t}, \mathbf{X}_{t}=\int_{0}^{t} e^{(t-s) A} F\left(\mathcal{X}_{\lfloor s\rfloor_{T / M}}\right) d s, \mathcal{X}_{t}=\int_{0}^{t} \mathcal{S}_{\lfloor s\rfloor_{T / M}, t} \mathbb{1}_{\left\{\mathcal{V}\left(\mathcal{X}_{\lfloor s\rfloor_{T / M}}\right) \leq M / T\right\}} F\left(\mathcal{X}_{\lfloor s\rfloor_{T / M}}\right) d s+\mathcal{O}_{t}$, and $\overline{\mathcal{X}}_{t}=\mathcal{X}_{t}-\mathcal{O}_{t}$.

\subsection{Regularity of the exact solution}

The next elementary lemma is a slightly modified version of Theorem 3.5 in Section 4.3 in Pazy [37].

Lemma 3.1. Let $T \in(0, \infty), \alpha \in(0,1]$, let $\left(W,\|\cdot\|_{W}\right)$ be an $\mathbb{R}$-Banach space, let $A: D(A) \subseteq W \rightarrow$ $W$ be a generator of an analytic semigroup with $\operatorname{spectrum}(A) \subseteq\{z \in \mathbb{C}: \operatorname{Re}(z)<0\}$, let $f:[0, T] \rightarrow$ $W$ be a continuous function which satisfies $\sup _{0 \leq s<t \leq T} \frac{s\|f(t)-\bar{f}(s)\|_{W}}{(t-s)^{\alpha}}<\infty$, and let $x:[0, T] \rightarrow W$ be the function with the property that for all $t \in[0, T]$ it holds that $x(t)=\int_{0}^{t} e^{(t-s) A} f(s) d s$. Then

(i) it holds for all $t \in[0, T]$ that $x(t) \in D(A)$,

(ii) it holds that the function $(0, T] \ni t \mapsto x(t) \in D(A)$ is continuous,

(iii) it holds that the function $[0, T] \ni t \mapsto x(t) \in W$ is continuous, 
(iv) it holds that the function $(0, T] \ni t \mapsto x(t) \in W$ is continuously differentiable, and

(v) it holds for all $t \in(0, T]$ that $x^{\prime}(t)=A x(t)+f(t)$.

Proof of Lemma 3.1. Throughout this proof assume w.l.o.g. that $W \neq\{0\}$ and let $K \in(0, \infty)$ be the real number which satisfies $K=\sup _{0 \leq s<u \leq T} \sup _{r \in\left\{0, \frac{\alpha}{2}, \frac{1}{2}, 1\right\}}\left[\left\|(-u A)^{r} e^{u A}\right\|_{L(W)}+\|(-u A)^{-r}\left(e^{u A}-\right.\right.$ $\left.\left.\operatorname{Id}_{W}\right)\left\|_{L(W)}+\right\| f(u) \|_{W}+\frac{s\|f(u)-f(s)\|_{W}}{(u-s)^{\alpha}}\right]$. The assumption that $A: D(A) \subseteq W \rightarrow W$ is a generator of an analytic semigroup with $\operatorname{spectrum}(A) \subseteq\{z \in \mathbb{C}: \operatorname{Re}(z)<0\}$ and the assumption that $f:[0, T] \rightarrow W$ is a continuous function with the property that $\sup _{0 \leq s<u \leq T} \frac{s\|f(u)-f(s)\|_{W}}{(u-s)^{\alpha}}<\infty$ assure that such a real number does indeed exist. Next note that for all $t \in(0, T], \varepsilon \in(0, t)$ it holds that

$$
\begin{aligned}
x(t) & =e^{(t-\varepsilon) A} \int_{0}^{\varepsilon} e^{(\varepsilon-s) A} f(s) d s+\int_{\varepsilon}^{t} e^{(t-s) A} f(s) d s \\
& =e^{(t-\varepsilon) A} x(\varepsilon)+\int_{\varepsilon}^{t} e^{(t-s) A}[f(s)-f(t)] d s+\int_{0}^{(t-\varepsilon)} e^{s A} f(t) d s \\
& =e^{(t-\varepsilon) A} x(\varepsilon)+\int_{\varepsilon}^{t} e^{(t-s) A}[f(s)-f(t)] d s+A^{-1}\left(e^{(t-\varepsilon) A}-\operatorname{Id}_{W}\right) f(t) .
\end{aligned}
$$

Moreover, observe that for all $t \in(0, T], \varepsilon \in(0, t)$ it holds that

$$
\begin{aligned}
\int_{\varepsilon}^{t}\left\|A e^{(t-s) A}[f(s)-f(t)]\right\|_{W} d s & \leq \int_{\varepsilon}^{t}\left\|A e^{(t-s) A}\right\|_{L(W)}\|f(s)-f(t)\|_{W} d s \\
& \leq \frac{K^{2}}{\varepsilon} \int_{\varepsilon}^{t}(t-s)^{(\alpha-1)} d s \leq \frac{K^{2} t^{\alpha}}{\alpha \varepsilon}<\infty .
\end{aligned}
$$

Combining the fact that $x(0)=0,(23)$, and (24) proves (ii). In addition, note that for all $t \in(0, T]$, $\varepsilon \in(0, t), t_{1}, t_{2} \in(\varepsilon, T]$ with $t_{1} \leq t \leq t_{2}$ it holds that

$$
\begin{aligned}
x & \left(t_{2}\right)-x\left(t_{1}\right) \\
= & \int_{t_{1}}^{t_{2}} e^{\left(t_{2}-s\right) A} f(s) d s+\left(e^{\left(t_{2}-t_{1}\right) A}-\operatorname{Id}_{W}\right) \int_{0}^{t_{1}} e^{\left(t_{1}-s\right) A} f(s) d s \\
= & \int_{t_{1}}^{t_{2}} e^{\left(t_{2}-s\right) A}\left[f(s)-f\left(t_{2}\right)\right] d s+\int_{t_{1}}^{t_{2}} e^{\left(t_{2}-s\right) A} f\left(t_{2}\right) d s+\left(e^{\left(t_{2}-t_{1}\right) A}-\operatorname{Id}_{W}\right) \int_{0}^{\varepsilon} e^{\left(t_{1}-s\right) A} f(s) d s \\
& +\left(e^{\left(t_{2}-t_{1}\right) A}-\operatorname{Id}_{W}\right) \int_{\varepsilon}^{t_{1}} e^{\left(t_{1}-s\right) A} f\left(t_{1}\right) d s+\left(e^{\left(t_{2}-t_{1}\right) A}-\operatorname{Id}_{W}\right) \int_{\varepsilon}^{t_{1}} e^{\left(t_{1}-s\right) A}\left[f(s)-f\left(t_{1}\right)\right] d s .
\end{aligned}
$$

Therefore, we obtain that for all $t \in(0, T], \varepsilon \in(0, t), t_{1}, t_{2} \in(\varepsilon, T]$ with $t_{1} \leq t \leq t_{2}$ it holds that

$$
\begin{aligned}
& x\left(t_{2}\right)-x\left(t_{1}\right) \\
& =\int_{t_{1}}^{t_{2}} e^{\left(t_{2}-s\right) A}\left[f(s)-f\left(t_{2}\right)\right] d s+A^{-1}\left(e^{\left(t_{2}-t_{1}\right) A}-\operatorname{Id}_{W}\right)\left[f\left(t_{2}\right)-f(t)\right] \\
& +A^{-1} e^{\left(t_{1}-\varepsilon\right) A}\left(e^{\left(t_{2}-t_{1}\right) A}-\operatorname{Id}_{W}\right) f(t)+A^{-1}\left(e^{\left(t_{1}-\varepsilon\right) A}-\operatorname{Id}_{W}\right)\left(e^{\left(t_{2}-t_{1}\right) A}-\operatorname{Id}_{W}\right)\left[f\left(t_{1}\right)-f(t)\right] \\
& +\left(e^{\left(t_{2}-t_{1}\right) A}-\operatorname{Id}_{W}\right) e^{\left(t_{1}-\varepsilon\right) A} \int_{0}^{\varepsilon} e^{(\varepsilon-s) A} f(s) d s+\left(e^{\left(t_{2}-t_{1}\right) A}-\operatorname{Id}_{W}\right) \int_{\varepsilon}^{t_{1}} e^{\left(t_{1}-s\right) A}\left[f(s)-f\left(t_{1}\right)\right] d s .
\end{aligned}
$$


This implies that for all $t \in(0, T], \varepsilon \in(0, t), t_{1}, t_{2} \in(\varepsilon, T]$ with $t_{1} \leq t \leq t_{2}$ it holds that

$$
\begin{aligned}
\| & A\left(x\left(t_{2}\right)-x\left(t_{1}\right)\right) \|_{W} \\
\leq & \int_{t_{1}}^{t_{2}}\left\|A e^{\left(t_{2}-s\right) A}\right\|_{L(W)}\left\|f(s)-f\left(t_{2}\right)\right\|_{W} d s+\left\|e^{\left(t_{2}-t_{1}\right) A}-\operatorname{Id}_{W}\right\|_{L(W)}\left\|f\left(t_{2}\right)-f(t)\right\|_{W} \\
& +\left\|e^{\left(t_{1}-\varepsilon\right) A}\right\|_{L(W)}\left\|\left(e^{\left(t_{2}-t_{1}\right) A}-\operatorname{Id}_{W}\right) f(t)\right\|_{W} \\
& +\left\|e^{\left(t_{1}-\varepsilon\right) A}-\operatorname{Id}_{W}\right\|_{L(W)}\left\|e^{\left(t_{2}-t_{1}\right) A}-\operatorname{Id}_{W}\right\|_{L(W)}\left\|f\left(t_{1}\right)-f(t)\right\|_{W} \\
& +\left\|(-A)^{-\frac{1}{2}}\left(e^{\left(t_{2}-t_{1}\right) A}-\operatorname{Id}_{W}\right)\right\|_{L(W)}\left\|A e^{\left(t_{1}-\varepsilon\right) A}\right\|_{L(W)} \int_{0}^{\varepsilon}\left\|(-A)^{\frac{1}{2}} e^{(\varepsilon-s) A}\right\|_{L(W)}\|f(s)\|_{W} d s \\
& +\left\|(-A)^{-\frac{\alpha}{2}}\left(e^{\left(t_{2}-t_{1}\right) A}-\operatorname{Id}_{W}\right)\right\|_{L(W)} \int_{\varepsilon}^{t_{1}}\left\|(-A)^{\left(1+\frac{\alpha}{2}\right)} e^{\left(t_{1}-s\right) A}\right\|_{L(W)}\left\|f(s)-f\left(t_{1}\right)\right\|_{W} d s .
\end{aligned}
$$

The fact that $K \geq 1$ hence assures that for all $t \in(0, T], \varepsilon \in(0, t), t_{1}, t_{2} \in(\varepsilon, T]$ with $t_{1} \leq t \leq t_{2}$ it holds that

$$
\begin{aligned}
\| & A\left(x\left(t_{2}\right)-x\left(t_{1}\right)\right) \|_{W} \\
\leq & \frac{K^{2}}{t_{1}} \int_{t_{1}}^{t_{2}}\left|t_{2}-s\right|^{(\alpha-1)} d s+\frac{K^{2}}{t}\left|t_{2}-t\right|^{\alpha}+K\left\|\left(e^{\left(t_{2}-t_{1}\right) A}-\operatorname{Id}_{W}\right) f(t)\right\|_{W}+\frac{K^{3}}{t_{1}}\left|t-t_{1}\right|^{\alpha} \\
& \left.+\frac{K^{3}\left|t_{2}-t_{1}\right|^{\frac{1}{2}}}{\left(t_{1}-\varepsilon\right)} \sup _{s \in(0, \varepsilon)}\|f(s)\|_{W}\right] \int_{0}^{\varepsilon}(\varepsilon-s)^{-\frac{1}{2}} d s \\
& +K^{2}\left|t_{2}-t_{1}\right|^{\frac{\alpha}{2}} \int_{\varepsilon}^{t_{1}}\left\|(-A) e^{\frac{\left(t_{1}-s\right)}{2}} A\right\|_{L(W)}\left\|(-A)^{\frac{\alpha}{2}} e^{\frac{\left(t_{1}-s\right)}{2}} A\right\|_{L(W)} \frac{\left|t_{1}-s\right|^{\alpha}}{s} d s \\
\leq & \frac{K^{2}\left|t_{2}-t_{1}\right|^{\alpha}}{\alpha \varepsilon}+\frac{K^{2}\left|t_{2}-t\right|^{\alpha}}{\varepsilon}+K\left\|\left(e^{\left(t_{2}-t_{1}\right) A}-\operatorname{Id}_{W}\right) f(t)\right\|_{W}+\frac{K^{3}\left|t-t_{1}\right|^{\alpha}}{\varepsilon} \\
& +\frac{2 K^{4} \varepsilon^{\frac{1}{2}}\left|t_{2}-t_{1}\right|^{\frac{1}{2}}}{\left(t_{1}-\varepsilon\right)}+\frac{4 K^{4}\left|t_{2}-t_{1}\right|^{\frac{\alpha}{2}}}{\varepsilon} \int_{\varepsilon}^{t_{1}}\left|t_{1}-s\right|^{\left(\frac{\alpha}{2}-1\right)} d s \\
\leq & \frac{\left(3 K^{3}+8 K^{4} T^{\frac{\alpha}{2}}\right)\left|t_{2}-t_{1}\right|^{\alpha}}{\alpha \varepsilon}+K\left\|\left(e^{\left(t_{2}-t_{1}\right) A}-\operatorname{Id}_{W}\right) f(t)\right\|_{W}+\frac{2 K^{4} \sqrt{T}\left|t_{2}-t_{1}\right|^{\frac{1}{2}}}{\left(t_{1}-\varepsilon\right)} .
\end{aligned}
$$

This ensures that for all $t \in(0, T], \varepsilon \in(0, t)$ it holds that

$$
\limsup _{\substack{\left(t_{1}, t_{2}\right) \rightarrow(t, t),\left(t_{1}, t_{2}\right) \in(\varepsilon, t] \times[t, T]}}\left\|A\left(x\left(t_{2}\right)-x\left(t_{1}\right)\right)\right\|_{W}=0 .
$$

Therefore, we obtain that the function $(0, T] \ni t \mapsto x(t) \in D(A)$ is continuous. Moreover, note that 
for all $t \in(0, T], \varepsilon \in(0, t), t_{1}, t_{2} \in(\varepsilon, T]$ with $t_{1} \leq t \leq t_{2}$ it holds that

$$
\begin{aligned}
x & \left(t_{2}\right)-x\left(t_{1}\right)-A x(t)\left(t_{2}-t_{1}\right)-f(t)\left(t_{2}-t_{1}\right) \\
= & \left(e^{\left(t_{2}-t_{1}\right) A}-\operatorname{Id}_{W}\right) x\left(t_{1}\right)-A x(t)\left(t_{2}-t_{1}\right)+\int_{t_{1}}^{t_{2}} e^{\left(t_{2}-s\right) A} f(s) d s-f(t)\left(t_{2}-t_{1}\right) \\
= & \left(e^{\left(t_{2}-t_{1}\right) A}-\operatorname{Id}_{W}-A\left(t_{2}-t_{1}\right)\right) x(t)+\left(e^{\left(t_{2}-t_{1}\right) A}-\operatorname{Id}_{W}\right)\left(x\left(t_{1}\right)-x(t)\right) \\
& +\int_{t_{1}}^{t_{2}} e^{\left(t_{2}-s\right) A}[f(s)-f(t)] d s+\int_{t_{1}}^{t_{2}}\left(e^{\left(t_{2}-s\right) A}-\operatorname{Id}_{W}\right) f(t) d s \\
= & \int_{0}^{\left(t_{2}-t_{1}\right)}\left(e^{s A}-\operatorname{Id}_{W}\right) A x(t) d s+\int_{0}^{\left(t_{2}-t_{1}\right)} e^{s A} A\left(x\left(t_{1}\right)-x(t)\right) d s \\
& +\int_{t_{1}}^{t_{2}} e^{\left(t_{2}-s\right) A}[f(s)-f(t)] d s+\int_{0}^{\left(t_{2}-t_{1}\right)}\left(e^{s A}-\operatorname{Id}_{W}\right) f(t) d s .
\end{aligned}
$$

This shows that for all $t \in(0, T], \varepsilon \in(0, t),\left(t_{1}, t_{2}\right) \in((\varepsilon, t] \times[t, T]) \backslash\{(t, t)\}$ it holds that

$$
\begin{aligned}
& \frac{\left\|x\left(t_{2}\right)-x\left(t_{1}\right)-A x(t)\left(t_{2}-t_{1}\right)-f(t)\left(t_{2}-t_{1}\right)\right\|_{W}}{\left(t_{2}-t_{1}\right)} \\
& \leq \sup _{s \in\left(0, t_{2}-t_{1}\right)}\left\|\left(e^{s A}-\operatorname{Id}_{W}\right) A x(t)\right\|_{W}+\sup _{s \in\left(0, t_{2}-t_{1}\right)}\left\|e^{s A}\right\|_{L(W)}\left\|A\left(x\left(t_{1}\right)-x(t)\right)\right\|_{W} \\
& \quad+\int_{t_{1}}^{t_{2}} \frac{\left\|e^{\left(t_{2}-s\right) A}\right\|_{L(W)}\|f(s)-f(t)\|_{W}}{\left(t_{2}-t_{1}\right)} d s+\sup _{s \in\left(0, t_{2}-t_{1}\right)}\left\|\left(e^{s A}-\operatorname{Id}_{W}\right) f(t)\right\|_{W} \\
& \leq \sup _{s \in\left(0, t_{2}-t_{1}\right)}\left\|\left(e^{s A}-\operatorname{Id}_{W}\right) A x(t)\right\|_{W}+K\left\|A\left(x\left(t_{1}\right)-x(t)\right)\right\|_{W}+\frac{K^{2}\left(t_{2}-t_{1}\right)^{\alpha}}{(1+\alpha) t_{1}} \\
& \quad+\sup _{s \in\left(0, t_{2}-t_{1}\right)}\left\|\left(e^{s A}-\operatorname{Id}_{W}\right) f(t)\right\|_{W} .
\end{aligned}
$$

This and (iii) ensure that for all $t \in(0, T], \varepsilon \in(0, t)$ it holds that

$$
\limsup _{\substack{\left(t_{1}, t_{2}\right) \rightarrow(t, t),\left(t_{1}, t_{2}\right) \in((\varepsilon, t] \times[t, T]) \backslash\{(t, t)\}}} \frac{\left\|x\left(t_{2}\right)-x\left(t_{1}\right)-A x(t)\left(t_{2}-t_{1}\right)-f(t)\left(t_{2}-t_{1}\right)\right\|_{W}}{\left(t_{2}-t_{1}\right)}=0 .
$$

Combining this with (iii) proves (iv) and (证). Finally, note that (iv) and the fact that

$$
\begin{aligned}
\underset{s \searrow 0}{\limsup \|x(s)-x(0)\|_{W}} & =\underset{s \searrow 0}{\limsup }\left\|\int_{0}^{s} e^{(s-u) A} f(u) d u\right\|_{W} \\
& \leq \underset{s \searrow 0}{\limsup }\left[\int_{0}^{s}\left\|e^{(s-u) A}\right\|_{L(W)}\|f(u)\|_{W} d u\right] \leq K^{2}\left[\limsup _{s \searrow 0}\right]=0
\end{aligned}
$$

show (iii). The proof of Lemma 3.1 is thus completed.

Corollary 3.2. Assume the setting in Section 3.1. Then

(i) it holds for all $\omega \in \Omega, t \in[0, T]$ that $\bar{X}_{t}(\omega) \in D(A)$, 
(ii) it holds for every $\omega \in \Omega$ that the function $(0, T] \ni t \mapsto \bar{X}_{t}(\omega) \in D(A)$ is continuous,

(iii) it holds for every $\omega \in \Omega$ that the function $[0, T] \ni t \mapsto \bar{X}_{t}(\omega) \in H$ is continuous,

(iv) it holds for every $\omega \in \Omega$ that the function $(0, T] \ni t \mapsto \bar{X}_{t}(\omega) \in H$ is continuously differentiable, and

(v) it holds for all $\omega \in \Omega, t \in(0, T]$ that $\frac{d \bar{X}_{t}(\omega)}{d t}=A \bar{X}_{t}(\omega)+F\left(X_{t}(\omega)\right)$.

Proof of Corollary 3.2. Throughout this proof let $\kappa \in(0,1-\rho)$ be a real number. Next note that the assumptions that $A: D(A) \subseteq H \rightarrow H$ is a generator of an analytic semigroup with $\operatorname{spectrum}(A) \subseteq$ $\{z \in \mathbb{C}: \operatorname{Re}(z)<0\}$, that $X:[0, T] \times \Omega \rightarrow V$ has continuous sample paths, that $F: V \rightarrow H$ is continuous, that $O:[0, T] \times \Omega \rightarrow V$ satisfies $\forall \omega \in \Omega: \lim \sup _{r \searrow 0} \sup _{0 \leq s<t \leq T} \frac{s\left\|O_{t}(\omega)-O_{s}(\omega)\right\|_{V}}{(t-s)^{r}}<\infty$, and that $\sup _{t \in(0, T)} t^{\rho}\left\|e^{t A}\right\|_{L(H, V)}<\infty$ imply that there exist functions $\theta: \Omega \rightarrow(0,1], K: \Omega \rightarrow[0, \infty)$ such that for all $\omega \in \Omega$ it holds that

$$
\begin{aligned}
K(\omega)= & \sup _{t \in(0, T]}\left[\left\|(-t A)^{\kappa} e^{t A}\right\|_{L(H)}+\left\|(-t A)^{-\kappa}\left(e^{t A}-\operatorname{Id}_{H}\right)\right\|_{L(H)}+t^{\rho}\left\|e^{t A}\right\|_{L(H, V)}\right] \\
& +\sup _{0 \leq s<t \leq T}\left[\left\|X_{t}(\omega)\right\|_{V}+\left\|F\left(X_{t}(\omega)\right)\right\|_{H}+\frac{s\left\|O_{t}(\omega)-O_{s}(\omega)\right\|_{V}}{|t-s|^{\theta(\omega)}}\right] .
\end{aligned}
$$

Moreover, observe that for all $\omega \in \Omega, t_{1}, t_{2} \in(0, T]$ with $t_{1}<t_{2}$ it holds that

$$
\begin{aligned}
& \left\|X_{t_{2}}(\omega)-X_{t_{1}}(\omega)\right\|_{V} \\
\leq & \int_{t_{1}}^{t_{2}}\left\|e^{\left(t_{2}-s\right) A} F\left(X_{s}(\omega)\right)\right\|_{V} d s+\int_{0}^{t_{1}}\left\|\left(e^{\left(t_{2}-s\right) A}-e^{\left(t_{1}-s\right) A}\right) F\left(X_{s}(\omega)\right)\right\|_{V} d s \\
& +\left\|O_{t_{2}}(\omega)-O_{t_{1}}(\omega)\right\|_{V} \\
\leq & \int_{t_{1}}^{t_{2}}\left\|e^{\left(t_{2}-s\right) A}\right\|_{L(H, V)}\left\|F\left(X_{s}(\omega)\right)\right\|_{H} d s+\frac{K(\omega)\left(t_{2}-t_{1}\right)}{t_{1}} \\
& +\int_{0}^{t_{1}}\left\|e^{\frac{\left(t_{1}-s\right)}{2} A}\right\|_{L(H, V)}\left\|e^{\frac{\left(t_{1}-s\right)}{2} A}\left(e^{\left(t_{2}-t_{1}\right) A}-\operatorname{Id}_{H}\right)\right\|_{L(H)}\left\|F\left(X_{s}(\omega)\right)\right\|_{H} d s .
\end{aligned}
$$

This shows that for all $\omega \in \Omega, t_{1}, t_{2} \in(0, T]$ with $t_{1}<t_{2}$ it holds that

$$
\begin{aligned}
& \left\|X_{t_{2}}(\omega)-X_{t_{1}}(\omega)\right\|_{V} \\
& \leq|K(\omega)|^{2} \int_{t_{1}}^{t_{2}}\left(t_{2}-s\right)^{-\rho} d s+\frac{K(\omega)\left(t_{2}-t_{1}\right)^{\theta(\omega)}}{t_{1}} \\
& \quad+2^{\rho}|K(\omega)|^{2} \int_{0}^{t_{1}}\left(t_{1}-s\right)^{-\rho}\left\|(-A)^{\kappa} e^{\frac{\left(t_{1}-s\right)}{2}} A\right\|_{L(H)}\left\|(-A)^{-\kappa}\left(e^{\left(t_{2}-t_{1}\right) A}-\operatorname{Id}_{H}\right)\right\|_{L(H)} d s \\
& \leq \\
& \leq \frac{|K(\omega)|^{2}\left(t_{2}-t_{1}\right)^{(1-\rho)}}{(1-\rho)}+\frac{K(\omega)\left(t_{2}-t_{1}\right)^{\theta(\omega)}}{t_{1}}+2^{(\rho+\kappa)}|K(\omega)|^{4}\left(t_{2}-t_{1}\right)^{\kappa} \int_{0}^{t_{1}}\left(t_{1}-s\right)^{-(\rho+\kappa)} d s .
\end{aligned}
$$


Hence, we obtain that for all $\omega \in \Omega, t_{1}, t_{2} \in(0, T]$ with $t_{1}<t_{2}$ it holds that

$$
\begin{aligned}
& \left\|X_{t_{2}}(\omega)-X_{t_{1}}(\omega)\right\|_{V} \\
\leq & \frac{|K(\omega)|^{2}\left(t_{2}-t_{1}\right)^{(1-\rho)}}{(1-\rho)}+\frac{K(\omega)\left(t_{2}-t_{1}\right)^{\theta(\omega)}}{t_{1}}+\frac{2^{(\rho+\kappa)}|K(\omega)|^{4}\left|t_{1}\right|^{(1-\rho-\kappa)}\left(t_{2}-t_{1}\right)^{\kappa}}{(1-\rho-\kappa)} \\
= & \frac{\left(t_{2}-t_{1}\right)^{\min \{\kappa, \theta(\omega)\}}}{t_{1}}\left(\frac{|K(\omega)|^{2} t_{1}\left(t_{2}-t_{1}\right)^{(1-\rho-\min \{\kappa, \theta(\omega)\})}}{(1-\rho)}+K(\omega)\left(t_{2}-t_{1}\right)^{\max \{0, \theta(\omega)-\kappa\}}\right. \\
& \left.+\frac{2^{(\rho+\kappa)}|K(\omega)|^{4}\left|t_{1}\right|^{(2-\rho-\kappa)}\left(t_{2}-t_{1}\right)^{\max \{0, \kappa-\theta(\omega)\}}}{(1-\rho-\kappa)}\right) \\
\leq & \frac{\left(t_{2}-t_{1}\right)^{\min \{\kappa, \theta(\omega)\}}}{t_{1}}\left(\frac{|K(\omega)|^{2}(1+T)^{2}}{(1-\rho)}+K(\omega)(1+T)+\frac{2^{(\rho+\kappa)}|K(\omega)|^{4}(1+T)^{2}}{(1-\rho-\kappa)}\right) .
\end{aligned}
$$

Combining this with the fact that $\forall u, v \in V:\|F(u)-F(v)\|_{H}^{2} \leq L\|u-v\|_{V}^{2}\left(1+\|u\|_{V}^{\varphi}+\|v\|_{V}^{\varphi}\right)$ yields that for all $\omega \in \Omega, t_{1}, t_{2} \in(0, T]$ with $t_{1}<t_{2}$ it holds that

$$
\begin{aligned}
& \left\|F\left(X_{t_{2}}(\omega)\right)-F\left(X_{t_{1}}(\omega)\right)\right\|_{H} \\
& \leq \sqrt{L\left(1+\left\|X_{t_{2}}(\omega)\right\|_{V}^{\varphi}+\left\|X_{t_{1}}(\omega)\right\|_{V}^{\varphi}\right)}\left\|X_{t_{2}}(\omega)-X_{t_{1}}(\omega)\right\|_{V} \\
& \leq \frac{\left(t_{2}-t_{1}\right)^{\min \{\kappa, \theta(\omega)\}}}{t_{1}} \sqrt{L\left(1+2|K(\omega)|^{\varphi}\right)}(1+T)^{2}\left(\frac{|K(\omega)|^{2}}{(1-\rho)}+K(\omega)+\frac{2^{(\rho+\kappa)}|K(\omega)|^{4}}{(1-\rho-\kappa)}\right) .
\end{aligned}
$$

This and Lemma 3.1 complete the proof of Corollary 3.2 ,

\subsection{Regularity of the semilinear integrated version of the numerical approximation}

Lemma 3.3. Assume the setting in Section 3.1. Then

(i) it holds for all $\omega \in \Omega, t \in[0, T]$ that $\mathbf{X}_{t}(\omega) \in D(A)$,

(ii) it holds for every $\omega \in \Omega$ that the function $[0, T] \ni t \mapsto \mathbf{X}_{t}(\omega) \in D(A)$ is continuous,

(iii) it holds for every $\omega \in \Omega$ that the function $[0, T] \ni t \mapsto \mathbf{X}_{t}(\omega) \in H$ is Lipschitz continuous,

(iv) it holds for every $\omega \in \Omega$ that the function $[0, T] \backslash\left\{0, \frac{T}{M}, \frac{2 T}{M}, \ldots, T\right\} \ni t \mapsto \mathbf{X}_{t}(\omega) \in H$ is continuously differentiable,

(v) it holds for all $\omega \in \Omega, t \in[0, T] \backslash\left\{0, \frac{T}{M}, \frac{2 T}{M}, \ldots, T\right\}$ that $\frac{d \mathbf{X}_{t}(\omega)}{d t}=A \mathbf{X}_{t}(\omega)+F\left(\mathcal{X}_{\lfloor t\rfloor_{T / M}}(\omega)\right)$, and

(vi) it holds for all $\omega \in \Omega, t \in[0, T]$ that $\mathbf{X}_{t}(\omega)=\int_{0}^{t}\left[A \mathbf{X}_{s}(\omega)+F\left(\mathcal{X}_{\lfloor s\rfloor_{T / M}}(\omega)\right)\right] d s$.

Proof of Lemma 3.3. Throughout this proof assume w.l.o.g. that $H \neq\{0\}$. Next note that the assumption that $A: D(A) \subseteq H \rightarrow H$ is a generator of an analytic semigroup with $\operatorname{spectrum}(A) \subseteq$ 
$\{z \in \mathbb{C}: \operatorname{Re}(z)<0\}$ ensures that there exists a function $K: \Omega \rightarrow(0, \infty)$ such that for all $\omega \in \Omega$ it holds that

$$
K(\omega)=\sup _{t \in\left\{0, \frac{T}{M}, \ldots, T\right\}}\left\|F\left(\mathcal{X}_{t}(\omega)\right)\right\|_{H}+\sup _{t \in(0, T]} \sup _{r \in\{0,1\}}\left[\left\|(-t A)^{r} e^{t A}\right\|_{L(H)}+\left\|(-t A)^{-r}\left(e^{t A}-\operatorname{Id}_{H}\right)\right\|_{L(H)}\right] .
$$

Moreover, observe that for all $\omega \in \Omega, t \in[0, T]$ it holds that

$$
\begin{aligned}
& \mathbf{X}_{t}(\omega)=\int_{\lfloor t\rfloor_{T / M}}^{t} e^{(t-s) A} F\left(\mathcal{X}_{\lfloor t\rfloor_{T / M}}(\omega)\right) d s+\sum_{l=0}^{\frac{M}{T}\lfloor t\rfloor_{T / M}-1} \int_{\frac{l T}{M}}^{\frac{(l+1) T}{M}} e^{(t-s) A} F\left(\mathcal{X}_{\lfloor s\rfloor_{T / M}}(\omega)\right) d s \\
& =A^{-1}\left(e^{\left(t-\lfloor t\rfloor_{T / M}\right) A}-\operatorname{Id}_{H}\right) F\left(\mathcal{X}_{\lfloor t\rfloor_{T / M}}(\omega)\right)+A^{-1} \sum_{l=0}^{\frac{M}{T}\lfloor t\rfloor_{T / M}-1} e^{\left(t-(l+1) \frac{T}{M}\right) A}\left(e^{\frac{T}{M} A}-\operatorname{Id}_{H}\right) F\left(\mathcal{X}_{\frac{l T}{M}}(\omega)\right) .
\end{aligned}
$$

This implies that for all $\omega \in \Omega, t \in[0, T]$ it holds that $\mathbf{X}_{t}(\omega) \in D(A)$. Furthermore, note that for all $\omega \in \Omega, s, t \in[0, T]$ with $s \leq t$ it holds that

$$
\begin{aligned}
\mathbf{X}_{t}(\omega)-\mathbf{X}_{s}(\omega) & \int_{\lfloor t\rfloor_{T / M}}^{t} e^{(t-u) A} F\left(\mathcal{X}_{\lfloor t\rfloor_{T / M}}(\omega)\right) d u-\int_{\lfloor s\rfloor_{T / M}}^{s} e^{(s-u) A} F\left(\mathcal{X}_{\lfloor s\rfloor_{T / M}}(\omega)\right) d u \\
& +\int_{\lfloor s\rfloor_{T / M}}^{\lfloor t\rfloor_{T / M}} e^{(t-u) A} F\left(\mathcal{X}_{\lfloor u\rfloor_{T / M}}(\omega)\right) d u+\left(e^{(t-s) A}-\operatorname{Id}_{H}\right) \int_{0}^{\lfloor s\rfloor_{T / M}} e^{(s-u) A} F\left(\mathcal{X}_{\lfloor u\rfloor_{T / M}}(\omega)\right) d u \\
= & A^{-1}\left(e^{\left(t-\lfloor t\rfloor_{T / M}\right) A}-\operatorname{Id}_{H}\right)\left(F\left(\mathcal{X}_{\lfloor t\rfloor_{T / M}}(\omega)\right)-F\left(\mathcal{X}_{\lfloor s\rfloor_{T / M}}(\omega)\right)\right) \\
& +A^{-1}\left(e^{\left(t-\lfloor t\rfloor_{T / M}\right) A}-e^{\left(s-\lfloor s\rfloor_{T / M}\right) A}\right) F\left(\mathcal{X}_{\lfloor s\rfloor_{T / M}}(\omega)\right) \\
& +A^{-1} \sum_{l=\frac{M}{T}\lfloor s\rfloor_{T / M}}^{\frac{M}{T}\lfloor\rfloor_{T / M}-1} e^{\left(t-(l+1) \frac{T}{M}\right) A}\left(e^{\frac{T}{M} A}-\operatorname{Id}_{H}\right) F\left(\mathcal{X}_{\frac{l T}{M}}(\omega)\right) \\
& +A^{-1}\left(e^{(t-s) A}-\operatorname{Id}_{H}\right) \sum_{l=0}^{\frac{M}{T}\lfloor s\rfloor_{T / M}-1} e^{\left(s-(l+1) \frac{T}{M}\right) A}\left(e^{\frac{T}{M} A}-\operatorname{Id}_{H}\right) F\left(\mathcal{X}_{\frac{l T}{M}}(\omega)\right) .
\end{aligned}
$$


This shows that for all $\omega \in \Omega, s, t \in[0, T]$ with $s \leq t$ it holds that

$$
\begin{aligned}
\| & A\left(\mathbf{X}_{t}(\omega)-\mathbf{X}_{s}(\omega)\right) \|_{H} \\
\leq & \left\|e^{\left(t-\lfloor t\rfloor_{T / M}\right) A}-\operatorname{Id}_{H}\right\|_{L(H)}\left\|F\left(\mathcal{X}_{\lfloor t\rfloor_{T / M}}(\omega)\right)-F\left(\mathcal{X}_{\lfloor s\rfloor_{T / M}}(\omega)\right)\right\|_{H} \\
& +\left\|e^{\min \left\{t-\lfloor t\rfloor_{T / M}, s-\lfloor s\rfloor_{T / M}\right\} A}\right\|_{L(H)}\left\|\left(e^{\left|t-s-\left(\lfloor t\rfloor_{T / M}-\lfloor s\rfloor_{T / M}\right)\right| A}-\operatorname{Id}_{H}\right) F\left(\mathcal{X}_{\lfloor s\rfloor_{T / M}}(\omega)\right)\right\|_{H} \\
& +\sum_{l=\frac{M}{T}\lfloor s\rfloor_{T / M}}{ }^{\frac{M}{T}\lfloor t\rfloor_{T / M}-1}\left\|e^{\left(t-(l+1) \frac{T}{M}\right) A}\right\|_{L(H)}\left\|e^{\frac{T}{M} A}-\operatorname{Id}_{H}\right\|_{L(H)}\left\|F\left(\mathcal{X}_{\frac{l T}{M}}(\omega)\right)\right\|_{H} \\
& +\sum_{l=0}^{\frac{M}{T}\lfloor s\rfloor_{T / M}-1}\left\|e^{\left(s-(l+1) \frac{T}{M}\right) A}\right\|_{L(H)}\left\|e^{\frac{T}{M} A}-\operatorname{Id}_{H}\right\|_{L(H)}\left\|\left(e^{(t-s) A}-\operatorname{Id}_{H}\right) F\left(\mathcal{X}_{\frac{l T}{M}}(\omega)\right)\right\|_{H} \\
\leq & K(\omega)\left\|F\left(\mathcal{X}_{\lfloor t\rfloor_{T / M}}(\omega)\right)-F\left(\mathcal{X}_{\lfloor s\rfloor_{T / M}}(\omega)\right)\right\|_{H} \\
& +K(\omega)\left\|\left(e^{\left|t-s-\left(\lfloor t\rfloor_{T / M}-\lfloor s\rfloor_{T / M}\right)\right| A}-\operatorname{Id}_{H}\right) F\left(\mathcal{X}_{\lfloor s\rfloor_{T / M}}(\omega)\right)\right\|_{H} \\
& +\frac{M|K(\omega)|^{3}}{T}\left(\lfloor t\rfloor_{T / M}-\lfloor s\rfloor_{T / M}\right)+\sup _{l \in\{0,1,2, \ldots, M\}} M|K(\omega)|^{2}\left\|\left(e^{(t-s) A}-\operatorname{Id}_{H}\right) F\left(\mathcal{X}_{\frac{l T}{M}}(\omega)\right)\right\|_{H} .
\end{aligned}
$$

In particular, this implies that for all $\omega \in \Omega, s \in[0, T]$ it holds that

$$
\limsup _{\substack{t \rightarrow s, t \in[s, T]}}\left\|A\left(\mathbf{X}_{t}(\omega)-\mathbf{X}_{s}(\omega)\right)\right\|_{H}=0 .
$$

Moreover, (42) assures that for all $\omega \in \Omega, t \in[0, T] \backslash\left\{0, \frac{T}{M}, \frac{2 T}{M}, \ldots, T\right\}$ it holds that

$$
\limsup _{\substack{s \rightarrow t, s \in[0, t]}}\left\|A\left(\mathbf{X}_{t}(\omega)-\mathbf{X}_{s}(\omega)\right)\right\|_{H}=0 .
$$

Next note that (41) implies that for all $\omega \in \Omega, t \in\left\{\frac{T}{M}, \frac{2 T}{M}, \ldots, T\right\}, s \in\left(t-\frac{T}{M}, t\right]$ it holds that

$$
\begin{aligned}
\mathbf{X}_{t}(\omega)-\mathbf{X}_{s}(\omega)= & A^{-1}\left(\operatorname{Id}_{H}-e^{\left(s-\left(t-\frac{T}{M}\right)\right) A}\right) F\left(\mathcal{X}_{\left(t-\frac{T}{M}\right)}(\omega)\right)+A^{-1}\left(e^{\frac{T}{M} A}-\operatorname{Id}_{H}\right) F\left(\mathcal{X}_{\left(t-\frac{T}{M}\right)}(\omega)\right) \\
& +A^{-1}\left(e^{(t-s) A}-\operatorname{Id}_{H}\right) \sum_{l=0}^{\frac{t M}{T}-2} e^{\left(s-(l+1) \frac{T}{M}\right) A}\left(e^{\frac{T}{M} A}-\operatorname{Id}_{H}\right) F\left(\mathcal{X}_{\frac{l T}{M}}(\omega)\right) .
\end{aligned}
$$

This ensures that for all $\omega \in \Omega, t \in\left\{\frac{T}{M}, \frac{2 T}{M}, \ldots, T\right\}, s \in\left(t-\frac{T}{M}, t\right]$ it holds that

$$
\begin{aligned}
\| & A\left(\mathbf{X}_{t}(\omega)-\mathbf{X}_{s}(\omega)\right) \|_{H} \\
\leq & \left\|\left(e^{\frac{T}{M} A}-e^{\left(s-\left(t-\frac{T}{M}\right)\right) A}\right) F\left(\mathcal{X}_{\left(t-\frac{T}{M}\right)}(\omega)\right)\right\|_{H} \\
& +\sum_{l=0}^{\frac{t M}{T}-2}\left\|e^{\left(s-(l+1) \frac{T}{M}\right) A}\right\|_{L(H)}\left\|\left(e^{\frac{T}{M} A}-\operatorname{Id}_{H}\right)\right\|_{L(H)}\left\|\left(e^{(t-s) A}-\operatorname{Id}_{H}\right) F\left(\mathcal{X}_{\frac{l T}{M}}(\omega)\right)\right\|_{H} \\
\leq & \left\|e^{\left(s-\left(t-\frac{T}{M}\right)\right) A}\right\|_{L(H)}\left\|\left(e^{(t-s) A}-\operatorname{Id}_{H}\right) F\left(\mathcal{X}_{\left(t-\frac{T}{M}\right)}(\omega)\right)\right\|_{H} \\
& +\sup _{l \in\{0,1,2, \ldots, M\}}|K(\omega)|^{2}\left(\frac{t M}{T}-1\right)\left\|\left(e^{(t-s) A}-\operatorname{Id}_{H}\right) F\left(\mathcal{X}_{\frac{l T}{M}}(\omega)\right)\right\|_{H} \\
\leq & \sup _{l \in\{0,1,2, \ldots, M\}} K(\omega)(1+K(\omega)) M\left\|\left(e^{(t-s) A}-\operatorname{Id}_{H}\right) F\left(\mathcal{X}_{\frac{l T}{M}}(\omega)\right)\right\|_{H} .
\end{aligned}
$$


Hence, we obtain that for all $\omega \in \Omega, t \in\left\{\frac{T}{M}, \frac{2 T}{M}, \ldots, T\right\}$ it holds that

$$
\limsup _{\substack{s \rightarrow t, s \in(t-T / M, t]}}\left\|A\left(\mathbf{X}_{t}(\omega)-\mathbf{X}_{s}(\omega)\right)\right\|_{H}=0 .
$$

Combining (43), (44), and (47) proves for every $\omega \in \Omega$ that the function $[0, T] \ni t \mapsto \mathbf{X}_{t}(\omega) \in D(A)$ is continuous. Moreover, note that for all $\omega \in \Omega, s, t \in[0, T]$ with $s<t$ it holds that

$$
\begin{aligned}
\mathbf{X}_{t}(\omega)-\mathbf{X}_{s}(\omega) \\
=\int_{s}^{t} e^{(t-u) A} F\left(\mathcal{X}_{\lfloor u\rfloor_{T / M}}(\omega)\right) d u+\left(e^{(t-s) A}-\operatorname{Id}_{H}\right) \int_{\lfloor s\rfloor_{T / M}}^{s} e^{(s-u) A} F\left(\mathcal{X}_{\lfloor s\rfloor_{T / M}}(\omega)\right) d u \\
\quad+\left(e^{(t-s) A}-\operatorname{Id}_{H}\right) \int_{0}^{\lfloor s\rfloor_{T / M}} e^{(s-u) A} F\left(\mathcal{X}_{\lfloor u\rfloor_{T / M}}(\omega)\right) d u . \\
=\int_{s}^{t} e^{(t-u) A} F\left(\mathcal{X}_{\lfloor u\rfloor_{T / M}}(\omega)\right) d u+A^{-1}\left(e^{(t-s) A}-\operatorname{Id}_{H}\right)\left(e^{\left(s-\lfloor s\rfloor_{T / M}\right) A}-\operatorname{Id}_{H}\right) F\left(\mathcal{X}_{\lfloor s\rfloor_{T / M}}(\omega)\right) \\
\quad+A^{-1}\left(e^{(t-s) A}-\operatorname{Id}_{H}\right) \sum_{l=0}^{\frac{M}{T}\lfloor s\rfloor_{T / M}-1} e^{\left(s-(l+1) \frac{T}{M}\right) A}\left(e^{\frac{T}{M} A}-\operatorname{Id}_{H}\right) F\left(\mathcal{X}_{\frac{l T}{M}}(\omega)\right) .
\end{aligned}
$$

This shows that for all $\omega \in \Omega, s, t \in[0, T]$ with $s<t$ it holds that

$$
\begin{aligned}
\| & \mathbf{X}_{t}(\omega)-\mathbf{X}_{s}(\omega) \|_{H} \\
\leq & \int_{s}^{t}\left\|e^{(t-u) A}\right\|_{L(H)}\left\|F\left(\mathcal{X}_{\lfloor u\rfloor_{T / M}}(\omega)\right)\right\|_{H} d u \\
& +\left\|A^{-1}\left(e^{(t-s) A}-\operatorname{Id}_{H}\right)\right\|_{L(H)}\left\|e^{\left(s-\lfloor s\rfloor_{T / M}\right) A}-\operatorname{Id}_{H}\right\|_{L(H)}\left\|F\left(\mathcal{X}_{\lfloor s\rfloor_{T / M}}(\omega)\right)\right\|_{H} \\
& +\left\|A^{-1}\left(e^{(t-s) A}-\operatorname{Id}_{H}\right)\right\|_{L(H)} \sum_{l=0}^{\frac{M}{T}\lfloor s\rfloor_{T / M}-1}\left\|e^{\left(s-(l+1) \frac{T}{M}\right) A}\left(e^{\frac{T}{M} A}-\operatorname{Id}_{H}\right) F\left(\mathcal{X}_{\frac{l T}{M}}(\omega)\right)\right\|_{H} \\
\leq & (t-s)|K(\omega)|^{2}(1+K(\omega)) \\
& +(t-s)|K(\omega)| \sum_{l=0}^{\frac{M}{T}\lfloor s\rfloor_{T / M}-1}\left\|e^{\left(s-(l+1) \frac{T}{M}\right) A}\right\|_{L(H)}\left\|e^{\frac{T}{M} A}-\operatorname{Id}_{H}\right\|_{L(H)}\left\|F\left(\mathcal{X}_{\frac{l T}{M}}(\omega)\right)\right\|_{H} \\
\leq & (t-s)|K(\omega)|^{2}(1+K(\omega))+(t-s)|K(\omega)|^{4} M .
\end{aligned}
$$

Hence, we obtain for every $\omega \in \Omega$ that the function $[0, T] \ni t \mapsto \mathbf{X}_{t}(\omega) \in H$ is Lipschitz continuous. 
Furthermore, observe that for all $\omega \in \Omega, t_{1}, t_{2}, t \in[0, T]$ with $t_{1} \leq t \leq t_{2}$ it holds that

$$
\begin{aligned}
\mathbf{X}_{t_{2}}(\omega)-\mathbf{X}_{t_{1}}(\omega)-A \mathbf{X}_{t}(\omega)\left(t_{2}-t_{1}\right)-F\left(\mathcal{X}_{\lfloor t\rfloor_{T / M}}(\omega)\right)\left(t_{2}-t_{1}\right) \\
=\left(e^{\left(t_{2}-t_{1}\right) A}-\operatorname{Id}_{H}\right) \mathbf{X}_{t_{1}}(\omega)-A \mathbf{X}_{t}(\omega)\left(t_{2}-t_{1}\right)+\int_{t_{1}}^{t_{2}}\left[e^{\left(t_{2}-s\right) A} F\left(\mathcal{X}_{\lfloor s\rfloor_{T / M}}(\omega)\right)-F\left(\mathcal{X}_{\lfloor t\rfloor_{T / M}}(\omega)\right)\right] d s \\
=\left(e^{\left(t_{2}-t_{1}\right) A}-\operatorname{Id}_{H}-A\left(t_{2}-t_{1}\right)\right) \mathbf{X}_{t}(\omega)+\left(e^{\left(t_{2}-t_{1}\right) A}-\operatorname{Id}_{H}\right)\left(\mathbf{X}_{t_{1}}(\omega)-\mathbf{X}_{t}(\omega)\right) \\
\quad+\int_{t_{1}}^{t_{2}} e^{\left(t_{2}-s\right) A}\left[F\left(\mathcal{X}_{\lfloor s\rfloor_{T / M}}(\omega)\right)-F\left(\mathcal{X}_{\lfloor\rfloor_{T / M}}(\omega)\right)\right] d s+\int_{t_{1}}^{t_{2}}\left(e^{\left(t_{2}-s\right) A}-\operatorname{Id}_{H}\right) F\left(\mathcal{X}_{\lfloor\rfloor_{T / M}}(\omega)\right) d s \\
=\int_{0}^{\left(t_{2}-t_{1}\right)}\left(e^{s A}-\operatorname{Id}_{H}\right) A \mathbf{X}_{t}(\omega) d s+\int_{0}^{\left(t_{2}-t_{1}\right)} e^{s A} A\left(\mathbf{X}_{t_{1}}(\omega)-\mathbf{X}_{t}(\omega)\right) d s \\
\quad+\int_{t_{1}}^{t_{2}} e^{\left(t_{2}-s\right) A}\left[F\left(\mathcal{X}_{\lfloor s\rfloor_{T / M}}(\omega)\right)-F\left(\mathcal{X}_{\lfloor t\rfloor_{T / M}}(\omega)\right)\right] d s+\int_{0}^{\left(t_{2}-t_{1}\right)}\left(e^{s A}-\operatorname{Id}_{H}\right) F\left(\mathcal{X}_{\lfloor t\rfloor_{T / M}}(\omega)\right) d s .
\end{aligned}
$$

This implies that for all $\omega \in \Omega, t \in[0, T],\left(t_{1}, t_{2}\right) \in([0, t] \times[t, T]) \backslash\{(t, t)\}$ it holds that

$$
\begin{aligned}
& \frac{\left\|\mathbf{X}_{t_{2}}(\omega)-\mathbf{X}_{t_{1}}(\omega)-\left(t_{2}-t_{1}\right) A \mathbf{X}_{t}(\omega)-\left(t_{2}-t_{1}\right) F\left(\mathcal{X}_{\lfloor t\rfloor_{T / M}}(\omega)\right)\right\|_{H}}{\left(t_{2}-t_{1}\right)} \\
& \leq \sup _{s \in\left(0, t_{2}-t_{1}\right)}\left\|\left(e^{s A}-\operatorname{Id}_{H}\right) A \mathbf{X}_{t}(\omega)\right\|_{H}+\sup _{s \in\left(0, t_{2}-t_{1}\right)}\left\|e^{s A}\right\|_{L(H)}\left\|A\left(\mathbf{X}_{t_{1}}(\omega)-\mathbf{X}_{t}(\omega)\right)\right\|_{H} \\
& +\sup _{s \in\left(t_{1}, t_{2}\right)}\left\|e^{\left(t_{2}-s\right) A}\right\|_{L(H)}\left\|F\left(\mathcal{X}_{\lfloor s\rfloor_{T / M}}(\omega)\right)-F\left(\mathcal{X}_{\lfloor t\rfloor_{T / M}}(\omega)\right)\right\|_{H} \\
& +\sup _{s \in\left(0, t_{2}-t_{1}\right)}\left\|\left(e^{s A}-\operatorname{Id}_{H}\right) F\left(\mathcal{X}_{\lfloor t\rfloor_{T / M}}(\omega)\right)\right\|_{H} \\
& \leq \sup _{s \in\left(0, t_{2}-t_{1}\right)}\left\|\left(e^{s A}-\operatorname{Id}_{H}\right) A \mathbf{X}_{t}(\omega)\right\|_{H}+K(\omega)\left\|A\left(\mathbf{X}_{t_{1}}(\omega)-\mathbf{X}_{t}(\omega)\right)\right\|_{H} \\
& +\sup _{s \in\left(t_{1}, t_{2}\right)} K(\omega)\left\|F\left(\mathcal{X}_{\lfloor s\rfloor_{T / M}}(\omega)\right)-F\left(\mathcal{X}_{\lfloor t\rfloor_{T / M}}(\omega)\right)\right\|_{H}+\sup _{s \in\left(0, t_{2}-t_{1}\right)}\left\|\left(e^{s A}-\operatorname{Id}_{H}\right) F\left(\mathcal{X}_{\lfloor t\rfloor_{T / M}}(\omega)\right)\right\|_{H} .
\end{aligned}
$$

This and (iii) ensure that for all $\omega \in \Omega, t \in[0, T] \backslash\left\{0, \frac{T}{M}, \frac{2 T}{M}, \ldots, T\right\}$ it holds that

$$
\frac{\left\|\mathbf{X}_{t_{2}}(\omega)-\mathbf{X}_{t_{1}}(\omega)-\left(t_{2}-t_{1}\right) A \mathbf{X}_{t}(\omega)-\left(t_{2}-t_{1}\right) F\left(\mathcal{X}_{\lfloor\rfloor_{T / M}}(\omega)\right)\right\|_{H}}{\left(t_{2}-t_{1}\right)}=0 .
$$

The proof of Lemma 3.3 is thus completed.

Lemma 3.4 (Temporal regularity). Assume the setting in Section 3.1 and let $\varrho \in[0,1-\rho)$, 
$t_{1}, t_{2} \in[0, T]$ with $t_{1}<t_{2}$. Then

$$
\begin{aligned}
\left\|\mathbf{X}_{t_{2}}-\mathbf{X}_{t_{1}}\right\|_{V} \leq & {\left[\sup _{s \in(0, T)} s^{\rho}\left\|e^{s A}\right\|_{L(H, V)}\right] \int_{t_{1}}^{t_{2}}\left(t_{2}-s\right)^{-\rho}\left\|F\left(\mathcal{X}_{\lfloor s\rfloor_{T / M}}\right)\right\|_{H} d s } \\
& +2^{(\rho+\varrho)}\left(t_{2}-t_{1}\right)^{\varrho}\left[\sup _{s \in(0, T)} s^{\rho}\left\|e^{s A}\right\|_{L(H, V)}\right]\left[\sup _{s \in(0, T)}\left\|(-s A)^{\varrho} e^{s A}\right\|_{L(H)}\right] \\
& \cdot\left[\sup _{s \in(0, T)}\left\|(-s A)^{-\varrho}\left(e^{s A}-\operatorname{Id}_{H}\right)\right\|_{L(H)}\right] \int_{0}^{t_{1}}\left(t_{1}-s\right)^{-(\rho+\varrho)} \| F\left(\mathcal{X}_{\left.\lfloor s\rfloor_{T / M}\right)} \|_{H} d s .\right.
\end{aligned}
$$

Proof of Lemma 3.4. Note that

$$
\begin{aligned}
\left\|\mathbf{X}_{t_{2}}-\mathbf{X}_{t_{1}}\right\|_{V} \\
\leq \int_{t_{1}}^{t_{2}}\left\|e^{\left(t_{2}-s\right) A} F\left(\mathcal{X}_{\lfloor s\rfloor_{T / M}}\right)\right\|_{V} d s+\int_{0}^{t_{1}}\left\|\left(e^{\left(t_{2}-t_{1}\right) A}-\operatorname{Id}_{H}\right) e^{\left(t_{1}-s\right) A} F\left(\mathcal{X}_{\lfloor s\rfloor_{T / M}}\right)\right\|_{V} d s \\
\leq\left[\sup _{s \in\left(0, t_{2}-t_{1}\right)} s^{\rho}\left\|e^{s A}\right\|_{L(H, V)}\right] \int_{t_{1}}^{t_{2}}\left(t_{2}-s\right)^{-\rho}\left\|F\left(\mathcal{X}_{\lfloor s\rfloor_{T / M}}\right)\right\|_{H} d s \\
+2^{\rho}\left[\sup _{s \in\left(0, t_{1}\right)} s^{\rho}\left\|e^{s A}\right\|_{L(H, V)}\right] \int_{0}^{t_{1}}\left(t_{1}-s\right)^{-\rho} \|\left(e^{\left(t_{2}-t_{1}\right) A}-\operatorname{Id}_{H}\right) e^{\frac{\left(t_{1}-s\right)}{2} A} F\left(\mathcal{X}_{\left.\lfloor s\rfloor_{T / M}\right) \|_{H} d s .}\right.
\end{aligned}
$$

This implies that

$$
\begin{aligned}
&\left\|\mathbf{X}_{t_{2}}-\mathbf{X}_{t_{1}}\right\|_{V} \\
& \leq {\left[\sup _{s \in(0, T)} s^{\rho}\left\|e^{s A}\right\|_{L(H, V)}\right] \int_{t_{1}}^{t_{2}}\left(t_{2}-s\right)^{-\rho}\left\|F\left(\mathcal{X}_{\lfloor s\rfloor_{T / M}}\right)\right\|_{H} d s } \\
&+2^{(\rho+\varrho)}\left(t_{2}-t_{1}\right)^{\varrho}\left[\sup _{s \in(0, T)} s^{\rho}\left\|e^{s A}\right\|_{L(H, V)}\right] \int_{0}^{t_{1}}\left(t_{1}-s\right)^{-(\rho+\varrho)} \\
& \cdot\left\|\left(-\left(t_{2}-t_{1}\right) A\right)^{-\varrho}\left(e^{\left(t_{2}-t_{1}\right) A}-\operatorname{Id}_{H}\right)\right\|_{L(H)}\left\|\left(-\frac{\left(t_{1}-s\right)}{2} A\right)^{\varrho} e^{\frac{\left(t_{1}-s\right)}{2} A}\right\|_{L(H)} \| F\left(\mathcal{X}_{\left.\lfloor s\rfloor_{T / M}\right) \|_{H} d s .}\right.
\end{aligned}
$$

The proof of Lemma 3.4 is thus completed.

\subsection{Analysis of the error between the numerical approximation and its semilinear integrated version}

Lemma 3.5. Assume the setting in Section 3.1 and let $\alpha \in(0, \infty), t \in(0, T]$. Then

$$
\begin{aligned}
\left\|\mathbf{X}_{t}-\overline{\mathcal{X}}_{t}\right\|_{V} \leq & \frac{T^{\alpha}}{M^{\alpha}}\left[\sup _{s \in(0, T)} s^{\rho}\left\|e^{s A}\right\|_{L(H, V)}\right] \int_{0}^{t}(t-s)^{-\rho}\left|\mathcal{V}\left(\mathcal{X}_{\lfloor s\rfloor_{T / M}}\right)\right|^{\alpha}\left\|F\left(\mathcal{X}_{\lfloor s\rfloor_{T / M}}\right)\right\|_{H} d s \\
& +\int_{0}^{t}\left\|e^{(t-s) A}-\mathcal{S}_{\lfloor s\rfloor_{T / M}, t}\right\|_{L(H, V)}\left\|F\left(\mathcal{X}_{\lfloor s\rfloor_{T / M}}\right)\right\|_{H} d s .
\end{aligned}
$$


Proof of Lemma 3.5. Note that the triangle inequality implies

$$
\begin{aligned}
\left\|\mathbf{X}_{t}-\overline{\mathcal{X}}_{t}\right\|_{V}= & \left\|\int_{0}^{t}\left(e^{(t-s) A} F\left(\mathcal{X}_{\lfloor s\rfloor_{T / M}}\right)-\mathcal{S}_{\lfloor s\rfloor_{T / M}, t} \mathbb{1}_{\left\{\mathcal{V}\left(\mathcal{X}_{\lfloor s\rfloor_{T / M}}\right) \leq M / T\right\}} F\left(\mathcal{X}_{\lfloor s\rfloor_{T / M}}\right)\right) d s\right\|_{V} \\
\leq & \int_{0}^{t} \| e^{(t-s) A} \mathbb{1}_{\left\{\mathcal{V}\left(\mathcal{X}_{\lfloor s\rfloor_{T / M}}\right)>M / T\right\}} F\left(\mathcal{X}_{\left.\lfloor s\rfloor_{T / M}\right) \|_{V}} d s\right. \\
& +\int_{0}^{t}\left\|\left(e^{(t-s) A}-\mathcal{S}_{\lfloor s\rfloor_{T / M}, t}\right) \mathbb{1}_{\left\{\mathcal{V}\left(\mathcal{X}_{\lfloor s\rfloor_{T / M}}\right) \leq M / T\right\}} F\left(\mathcal{X}_{\lfloor s\rfloor_{T / M}}\right)\right\|_{V} d s .
\end{aligned}
$$

Therefore, we obtain

$$
\begin{aligned}
\| & \mathbf{X}_{t}-\overline{\mathcal{X}}_{t} \|_{V} \\
\leq & \int_{0}^{t} \mathbb{1}_{\left\{\mathcal{V}\left(\mathcal{X}_{\lfloor s\rfloor_{T / M}}\right)>M / T\right\}}\left\|e^{(t-s) A}\right\|_{L(H, V)}\left\|F\left(\mathcal{X}_{\lfloor s\rfloor_{T / M}}\right)\right\|_{H} d s \\
& +\int_{0}^{t}\left\|e^{(t-s) A}-\mathcal{S}_{\lfloor s\rfloor_{T / M}, t}\right\|_{L(H, V)}\left\|F\left(\mathcal{X}_{\lfloor s\rfloor_{T / M}}\right)\right\|_{H} d s \\
\leq & {\left[\sup _{s \in(0, T)} s^{\rho}\left\|e^{s A}\right\|_{L(H, V)}\right] \int_{0}^{t}(t-s)^{-\rho} \mathbb{1}_{\left\{\left|\mathcal{V}\left(\mathcal{X}_{\lfloor s\rfloor_{T / M}}\right)\right|^{\alpha}>|M / T|^{\alpha}\right\}} \frac{T^{\alpha}}{M^{\alpha}}\left|\mathcal{V}\left(\mathcal{X}_{\lfloor s\rfloor_{T / M}}\right)\right|^{\alpha}\left\|F\left(\mathcal{X}_{\lfloor s\rfloor_{T / M}}\right)\right\|_{H} d s } \\
& +\int_{0}^{t}\left\|e^{(t-s) A}-\mathcal{S}_{\lfloor s\rfloor_{T / M}, t}\right\|_{L(H, V)} \| F\left(\mathcal{X}_{\left.\lfloor s\rfloor_{T / M}\right) \|_{H}} d s .\right.
\end{aligned}
$$

The proof of Lemma 3.5 is thus completed.

\subsection{Analysis of the error between the exact solution and the semilinear integrated version of the numerical approximation}

Lemma 3.6 (Error estimates based on the monotonicity assumption). Assume the setting in Section 3.1 and let $\kappa \in(2, \infty), t \in[0, T]$. Then

$$
\begin{aligned}
& \left\|\bar{X}_{t}-\mathbf{X}_{t}\right\|_{H}^{2} \leq \frac{1}{C(\kappa-2)} \int_{0}^{t} e^{\kappa C(t-s)}\left\|F\left(\mathbf{X}_{s}+O_{s}\right)-F\left(\mathcal{X}_{\lfloor s\rfloor_{T / M}}\right)\right\|_{H}^{2} d s \\
& \leq \frac{2^{[\varphi-1]^{+}} L}{C(\kappa-2)} \int_{0}^{t} e^{\kappa C(t-s)}\left(1+\left\|\mathbf{X}_{s}\right\|_{V}^{\varphi}+\left\|O_{s}\right\|_{V}^{\varphi}+\left\|\mathcal{X}_{\lfloor s\rfloor_{T / M}}\right\|_{V}^{\varphi}\right) \\
& \cdot\left(\left\|\mathbf{X}_{s}-\mathbf{X}_{\lfloor s\rfloor_{T / M}}\right\|_{V}+\left\|\mathbf{X}_{\lfloor s\rfloor_{T / M}}-\overline{\mathcal{X}}_{\lfloor s\rfloor_{T / M}}\right\|_{V}+\left\|O_{s}-O_{\lfloor s\rfloor_{T / M}}\right\|_{V}+\left\|O_{\lfloor s\rfloor_{T / M}}-\mathcal{O}_{\lfloor s\rfloor_{T / M}}\right\|_{V}\right)^{2} d s .
\end{aligned}
$$

Proof of Lemma 3.6. W.l.o.g. we assume that $t \in(0, T]$ (otherwise the proof is clear). Note that the fundamental theorem of calculus, Corollary [3.2, and Lemma 3.3 imply that for all $\varepsilon \in(0, t)$ it 
holds that

$$
\begin{aligned}
e^{-\kappa C t}\left\|\bar{X}_{t}-\mathbf{X}_{t}\right\|_{H}^{2}-e^{-\kappa C \varepsilon}\left\|\bar{X}_{\varepsilon}-\mathbf{X}_{\varepsilon}\right\|_{H}^{2} \\
=2 \int_{\varepsilon}^{t} e^{-\kappa C s}\left\langle\bar{X}_{s}-\mathbf{X}_{s}, A \bar{X}_{s}+F\left(X_{s}\right)-A \mathbf{X}_{s}-F\left(\mathcal{X}_{\lfloor s\rfloor_{T / M}}\right)\right\rangle_{H} d s \\
\quad-\kappa C \int_{\varepsilon}^{t} e^{-\kappa C s}\left\|\bar{X}_{s}-\mathbf{X}_{s}\right\|_{H}^{2} d s \\
=2 \int_{\varepsilon}^{t} e^{-\kappa C s}\left\langle\bar{X}_{s}-\mathbf{X}_{s}, A \bar{X}_{s}+F\left(X_{s}\right)-A \mathbf{X}_{s}-F\left(\mathbf{X}_{s}+O_{s}\right)\right\rangle_{H} d s \\
\quad+2 \int_{\varepsilon}^{t} e^{-\kappa C s}\left\langle\bar{X}_{s}-\mathbf{X}_{s}, F\left(\mathbf{X}_{s}+O_{s}\right)-F\left(\mathcal{X}_{\lfloor s\rfloor_{T / M}}\right)\right\rangle_{H} d s-\kappa C \int_{\varepsilon}^{t} e^{-\kappa C s}\left\|\bar{X}_{s}-\mathbf{X}_{s}\right\|_{H}^{2} d s .
\end{aligned}
$$

The assumption that $\forall x, y \in V$ with $x-y \in H_{1}:\langle x-y, A(x-y)+F(x)-F(y)\rangle_{H} \leq C\|x-y\|_{H}^{2}$ and the Cauchy-Schwarz inequality hence prove that for all $\varepsilon \in(0, t)$ it holds that

$$
\begin{aligned}
e^{-\kappa C t}\left\|\bar{X}_{t}-\mathbf{X}_{t}\right\|_{H}^{2}-e^{-\kappa C \varepsilon}\left\|\bar{X}_{\varepsilon}-\mathbf{X}_{\varepsilon}\right\|_{H}^{2} \\
=2 \int_{\varepsilon}^{t} e^{-\kappa C s}\left\langle X_{s}-\left(\mathbf{X}_{s}+O_{s}\right), A\left(X_{s}-\left[\mathbf{X}_{s}+O_{s}\right]\right)+F\left(X_{s}\right)-F\left(\mathbf{X}_{s}+O_{s}\right)\right\rangle_{H} d s \\
\quad+2 \int_{\varepsilon}^{t} e^{-\kappa C s}\left\langle\bar{X}_{s}-\mathbf{X}_{s}, F\left(\mathbf{X}_{s}+O_{s}\right)-F\left(\mathcal{X}_{\lfloor s\rfloor_{T / M}}\right)\right\rangle_{H} d s-\kappa C \int_{\varepsilon}^{t} e^{-\kappa C s}\left\|\bar{X}_{s}-\mathbf{X}_{s}\right\|_{H}^{2} d s \\
\leq 2 C \int_{\varepsilon}^{t} e^{-\kappa C s}\left\|X_{s}-\left(\mathbf{X}_{s}+O_{s}\right)\right\|_{H}^{2} d s-\kappa C \int_{\varepsilon}^{t} e^{-\kappa C s}\left\|\bar{X}_{s}-\mathbf{X}_{s}\right\|_{H}^{2} d s \\
\quad+2 \int_{\varepsilon}^{t} e^{-\kappa C s}\left\|\bar{X}_{s}-\mathbf{X}_{s}\right\|_{H} \| F\left(\mathbf{X}_{s}+O_{s}\right)-F\left(\mathcal{X}_{\left.\lfloor s\rfloor_{T / M}\right) \|_{H} d s .}\right.
\end{aligned}
$$

The fact that $\forall x, y \in \mathbb{R}: x y \leq \frac{x^{2}}{2}+\frac{y^{2}}{2}$ therefore shows that for all $\varepsilon \in(0, t)$ it holds that

$$
\begin{aligned}
e^{-\kappa C t}\left\|\bar{X}_{t}-\mathbf{X}_{t}\right\|_{H}^{2}-e^{-\kappa C \varepsilon}\left\|\bar{X}_{\varepsilon}-\mathbf{X}_{\varepsilon}\right\|_{H}^{2} \\
\leq C(2-\kappa) \int_{\varepsilon}^{t} e^{-\kappa C s}\left\|\bar{X}_{s}-\mathbf{X}_{s}\right\|_{H}^{2} d s \\
\quad+2 \int_{\varepsilon}^{t} e^{-\kappa C s}\left(\sqrt{C(\kappa-2)}\left\|\bar{X}_{s}-\mathbf{X}_{s}\right\|_{H}\right)\left(\frac{1}{\sqrt{C(\kappa-2)}} \| F\left(\mathbf{X}_{s}+O_{s}\right)-F\left(\mathcal{X}_{\left.\lfloor s\rfloor_{T / M}\right)} \|_{H}\right) d s\right. \\
\leq C(2-\kappa) \int_{\varepsilon}^{t} e^{-\kappa C s}\left\|\bar{X}_{s}-\mathbf{X}_{s}\right\|_{H}^{2} d s \\
\quad+\int_{\varepsilon}^{t} e^{-\kappa C s}\left[C(\kappa-2)\left\|\bar{X}_{s}-\mathbf{X}_{s}\right\|_{H}^{2}+\frac{1}{C(\kappa-2)}\left\|F\left(\mathbf{X}_{s}+O_{s}\right)-F\left(\mathcal{X}_{\left.\lfloor s\rfloor_{T / M}\right)}\right)\right\|_{H}^{2}\right] d s \\
=\frac{1}{C(\kappa-2)} \int_{\varepsilon}^{t} e^{-\kappa C s}\left\|F\left(\mathbf{X}_{s}+O_{s}\right)-F\left(\mathcal{X}_{\left.\lfloor s\rfloor_{T / M}\right)}\right)\right\|_{H}^{2} d s .
\end{aligned}
$$


Corollary 3.2 and Lemma 3.3 hence ensure

$$
\begin{aligned}
\left\|\bar{X}_{t}-\mathbf{X}_{t}\right\|_{H}^{2} & =\left\|\bar{X}_{t}-\mathbf{X}_{t}\right\|_{H}^{2}-\lim _{\substack{\varepsilon \rightarrow 0 \\
\varepsilon \in(0, t)}} e^{\kappa C(t-\varepsilon)}\left\|\bar{X}_{\varepsilon}-\mathbf{X}_{\varepsilon}\right\|_{H}^{2} \\
& \leq \frac{1}{C(\kappa-2)} \lim _{\substack{\varepsilon \rightarrow 0 \\
\varepsilon \in(0, t)}} \int_{\varepsilon}^{t} e^{\kappa C(t-s)}\left\|F\left(\mathbf{X}_{s}+O_{s}\right)-F\left(\mathcal{X}_{\lfloor s\rfloor_{T / M}}\right)\right\|_{H}^{2} d s \\
& =\frac{1}{C(\kappa-2)} \int_{0}^{t} e^{\kappa C(t-s)}\left\|F\left(\mathbf{X}_{s}+O_{s}\right)-F\left(\mathcal{X}_{\lfloor s\rfloor_{T / M}}\right)\right\|_{H}^{2} d s .
\end{aligned}
$$

Next observe that the triangle inequality implies that for all $s \in(0, t)$ it holds that

$$
\begin{aligned}
& \left\|\mathbf{X}_{s}+O_{s}-\mathcal{X}_{\lfloor s\rfloor_{T / M}}\right\|_{V} \\
& \leq\left\|\mathbf{X}_{s}-\mathbf{X}_{\lfloor s\rfloor_{T / M}}\right\|_{V}+\left\|\mathbf{X}_{\lfloor s\rfloor_{T / M}}+O_{s}-\mathcal{X}_{\lfloor s\rfloor_{T / M}}\right\|_{V} \\
& \leq\left\|\mathbf{X}_{s}-\mathbf{X}_{\lfloor s\rfloor_{T / M}}\right\|_{V}+\left\|\mathbf{X}_{\lfloor s\rfloor_{T / M}}-\left(\mathcal{X}_{\lfloor s\rfloor_{T / M}}-\mathcal{O}_{\lfloor s\rfloor_{T / M}}\right)\right\|_{V}+\left\|O_{s}-\mathcal{O}_{\lfloor s\rfloor_{T / M}}\right\|_{V} \\
& \leq\left\|\mathbf{X}_{s}-\mathbf{X}_{\lfloor s\rfloor_{T / M}}\right\|_{V}+\left\|\mathbf{X}_{\lfloor s\rfloor_{T / M}}-\overline{\mathcal{X}}_{\lfloor s\rfloor_{T / M}}\right\|_{V}+\left\|O_{s}-O_{\lfloor s\rfloor_{T / M}}\right\|_{V}+\left\|O_{\lfloor s\rfloor_{T / M}}-\mathcal{O}_{\lfloor s\rfloor_{T / M}}\right\|_{V} .
\end{aligned}
$$

Combining the fact that $\forall u, v \in V:\|F(u)-F(v)\|_{H}^{2} \leq L\|u-v\|_{V}^{2}\left(1+\|u\|_{V}^{\varphi}+\|v\|_{V}^{\varphi}\right)$ and the fact that $\forall r \in(0, \infty), x_{1}, x_{2} \in \mathbb{R}:\left|x_{1}+x_{2}\right|^{r} \leq 2^{[r-1]^{+}}\left(\left|x_{1}\right|^{r}+\left|x_{2}\right|^{r}\right)$ hence implies that for all $s \in(0, t)$ it holds that

$$
\begin{aligned}
& \left\|F\left(\mathbf{X}_{s}+O_{s}\right)-F\left(\mathcal{X}_{\lfloor s\rfloor_{T / M}}\right)\right\|_{H}^{2} \\
\leq & L\left\|\mathbf{X}_{s}+O_{s}-\mathcal{X}_{\lfloor s\rfloor_{T / M}}\right\|_{V}^{2}\left(1+\left\|\mathbf{X}_{s}+O_{s}\right\|_{V}^{\varphi}+\left\|\mathcal{X}_{\lfloor s\rfloor_{T / M}}\right\|_{V}^{\varphi}\right) \\
\leq & 2^{[\varphi-1]^{+}} L\left(1+\left\|\mathbf{X}_{s}\right\|_{V}^{\varphi}+\left\|O_{s}\right\|_{V}^{\varphi}+\left\|\mathcal{X}_{\lfloor s\rfloor_{T / M}}\right\|_{V}^{\varphi}\right) \\
& \cdot\left(\left\|\mathbf{X}_{s}-\mathbf{X}_{\lfloor s\rfloor_{T / M}}\right\|_{V}+\left\|\mathbf{X}_{\lfloor s\rfloor_{T / M}}-\overline{\mathcal{X}}_{\lfloor s\rfloor_{T / M}}\right\|_{V}+\left\|O_{s}-O_{\lfloor s\rfloor_{T / M}}\right\|_{V}+\left\|O_{\lfloor s\rfloor_{T / M}}-\mathcal{O}_{\lfloor s\rfloor_{T / M}}\right\|_{V}\right)^{2} .
\end{aligned}
$$

This and (63) complete the proof of Lemma 3.6 .

\section{Strong error estimates}

\subsection{Setting}

Let $T, C, \varphi \in(0, \infty), L \in[0, \infty), \rho \in[0,1)$, let $\left(H,\langle\cdot, \cdot\rangle_{H},\|\cdot\|_{H}\right)$ be a separable $\mathbb{R}$-Hilbert space, let $A: D(A) \subseteq H \rightarrow H$ be a generator of an analytic semigroup with spectrum $(A) \subseteq\{z \in$ $\mathbb{C}: \operatorname{Re}(z)<0\}$, let $\left(H_{r},\langle\cdot, \cdot\rangle_{H_{r}},\|\cdot\|_{H_{r}}\right), r \in \mathbb{R}$, be a family of interpolation spaces associated to $-A$ (cf., e.g., Definition 3.5.26 in [23]), let $\left(V,\|\cdot\|_{V}\right)$ be a separable $\mathbb{R}$-Banach space with $H_{1} \subseteq V \subseteq H$ densely and continuously, let $\mathcal{V} \in \mathcal{M}(\mathcal{B}(V), \mathcal{B}([0, \infty))),\left(\mathcal{S}^{M}\right)_{M \in \mathbb{N}} \subseteq \mathcal{M}\left(\mathcal{B}\left(\left\{(s, t) \in[0, T]^{2}: s<\right.\right.\right.$ t\}), $\mathcal{B}(L(H, V))), F \in \mathcal{C}(V, H)$ satisfy for all $x, y \in V$ with $x-y \in H_{1}$ that $\|F(x)-F(y)\|_{H}^{2} \leq$ $L\|x-y\|_{V}^{2}\left(1+\|x\|_{V}^{\varphi}+\|y\|_{V}^{\varphi}\right)$ and $\langle x-y, A(x-y)+F(x)-F(y)\rangle_{H} \leq C\|x-y\|_{H}^{2}$, assume that $\sup _{t \in(0, T)} t^{\rho}\left\|e^{t A}\right\|_{L(H, V)}<\infty$, let $(\Omega, \mathcal{F}, \mathbb{P})$ be a probability space, let $X:[0, T] \times \Omega \rightarrow V$ be a stochastic process with continuous sample paths, let $O, \bar{X}:[0, T] \times \Omega \rightarrow V$ be stochastic processes which satisfy for all $\omega \in \Omega$ that $\lim \sup _{r \searrow 0} \sup _{0 \leq s<t \leq T} \frac{s\left\|O_{t}(\omega)-O_{s}(\omega)\right\|_{V}}{(t-s)^{r}}<\infty$, let $\mathcal{O}^{M}, \mathcal{X}^{M}, \overline{\mathcal{X}}^{M}, \mathbf{X}^{M}:[0, T] \times$ 
$\Omega \rightarrow V, M \in \mathbb{N}$, be stochastic processes, and assume for all $t \in[0, T], M \in \mathbb{N}$ that $X_{t}=$ $\int_{0}^{t} e^{(t-s) A} F\left(X_{s}\right) d s+O_{t}, \bar{X}_{t}=X_{t}-O_{t}, \mathbf{X}_{t}^{M}=\int_{0}^{t} e^{(t-s) A} F\left(\mathcal{X}_{\lfloor s\rfloor_{T / M}^{M}}\right) d s, \overline{\mathcal{X}}_{t}^{M}=\mathcal{X}_{t}^{M}-\mathcal{O}_{t}^{M}$, and $\mathcal{X}_{t}^{M}=\int_{0}^{t} \mathcal{S}_{\lfloor s\rfloor_{T / M}, t}^{M} \mathbb{1}_{\left\{\mathcal{V}\left(\mathcal{X}_{\lfloor s\rfloor_{T / M}^{M}}^{M}\right) \leq M / T\right\}} F\left(\mathcal{X}_{\lfloor s\rfloor_{T / M}}^{M}\right) d s+\mathcal{O}_{t}^{M}$.

\subsection{Analysis of the error between the numerical approximation and its semilinear integrated version}

Lemma 4.1. Assume the setting in Section 4.1 and let $\alpha \in(0, \infty), p \in[1, \infty), t \in(0, T], M \in \mathbb{N}$. Then

$$
\begin{aligned}
& \left\|\mathbf{X}_{t}^{M}-\overline{\mathcal{X}}_{t}^{M}\right\|_{\mathcal{L}^{p}(\mathbb{P} ; V)} \leq\left(\int_{0}^{t}\left\|e^{(t-s) A}-\mathcal{S}_{\lfloor s\rfloor_{T / M}, t}^{M}\right\|_{L(H, V)} d s\right)\left[\sup _{s \in[0, T)}\left\|F\left(\mathcal{X}_{s}^{M}\right)\right\|_{\mathcal{L}^{p}(\mathbb{P} ; H)}\right] \\
& +\frac{T^{(1+\alpha-\rho)}}{(1-\rho) M^{\alpha}}\left[\sup _{s \in(0, T)} s^{\rho}\left\|e^{s A}\right\|_{L(H, V)}\right]\left[\sup _{s \in[0, T)}\left\|\mathcal{V}\left(\mathcal{X}_{s}^{M}\right)\right\|_{\mathcal{L}^{2 p \alpha(\mathbb{P} ; \mathbb{R})}}^{\alpha}\left\|F\left(\mathcal{X}_{s}^{M}\right)\right\|_{\mathcal{L}^{2 p}(\mathbb{P} ; H)}\right] .
\end{aligned}
$$

Proof of Lemma 4.1. Observe that Lemma 3.5 and Hölder's inequality imply

$$
\begin{aligned}
& \left\|\mathbf{X}_{t}^{M}-\overline{\mathcal{X}}_{t}^{M}\right\|_{\mathcal{L}^{p}(\mathbb{P} ; V)} \\
& \leq \frac{T^{\alpha}}{M^{\alpha}}\left[\sup _{s \in(0, T)} s^{\rho}\left\|e^{s A}\right\|_{L(H, V)}\right] \int_{0}^{t}(t-s)^{-\rho}\left\|\left|\mathcal{V}\left(\mathcal{X}_{\lfloor s\rfloor_{T / M}^{M}}^{M}\right)\right|^{\alpha}\right\| F\left(\mathcal{X}_{\lfloor s\rfloor_{T / M}^{M}}^{M}\left\|_{H}\right\|_{\mathcal{L}^{p}(\mathbb{P} ; \mathbb{R})} d s\right. \\
& +\int_{0}^{t}\left\|e^{(t-s) A}-\mathcal{S}_{\lfloor s\rfloor_{T / M}, t}^{M}\right\|_{L(H, V)}\left\|F\left(\mathcal{X}_{\lfloor s\rfloor_{T / M}}^{M}\right)\right\|_{\mathcal{L}^{p}(\mathbb{P} ; H)} d s \\
& \leq \frac{T^{\alpha}}{M^{\alpha}}\left[\sup _{s \in(0, T)} s^{\rho}\left\|e^{s A}\right\|_{L(H, V)}\right] \int_{0}^{t}(t-s)^{-\rho}\left\|\mathcal{V}\left(\mathcal{X}_{\lfloor s\rfloor_{T / M}^{M}}\right)\right\|_{\mathcal{L}^{2 p \alpha}(\mathbb{P} ; \mathbb{R})}^{\alpha}\left\|F\left(\mathcal{X}_{\lfloor s\rfloor_{T / M}}^{M}\right)\right\|_{\mathcal{L}^{2 p}(\mathbb{P} ; H)} d s \\
& +\left(\int_{0}^{t}\left\|e^{(t-s) A}-\mathcal{S}_{\lfloor s\rfloor_{T / M}, t}\right\|_{L(H, V)} d s\right)\left[\sup _{s \in[0, t)}\left\|F\left(\mathcal{X}_{s}^{M}\right)\right\|_{\mathcal{L}^{p}(\mathbb{P} ; H)}\right] .
\end{aligned}
$$

The proof of Lemma 4.1 is thus completed. 


\subsection{Analysis of the error between the exact solution and the semilinear integrated version of the numerical approximation}

Lemma 4.2. Assume the setting in Section 4.1 and let $\kappa \in(2, \infty), t \in[0, T], p \in[2, \infty), M \in \mathbb{N}$. Then

$$
\begin{aligned}
& \left\|\bar{X}_{t}-\mathbf{X}_{t}^{M}\right\|_{\mathcal{L}^{p(\mathbb{P} ; H)}} \\
& \leq \frac{e^{\kappa C T / 2} \sqrt{2^{[\varphi-1]^{+}} L}}{\sqrt{C(\kappa-2)}}\left[1+\sup _{s \in(0, T)}\left\|\mathbf{X}_{s}^{M}\right\|_{\mathcal{L}^{p \varphi}(\mathbb{P} ; V)}^{\varphi / 2}+\sup _{s \in(0, T)}\left\|O_{s}\right\|_{\mathcal{L}^{p \varphi}(\mathbb{P} ; V)}^{\varphi / 2}+\sup _{s \in[0, T)}\left\|\mathcal{X}_{s}^{M}\right\|_{\mathcal{L}^{p \varphi}(\mathbb{P} ; V)}^{\varphi / 2}\right] \\
& \cdot\left[\int _ { 0 } ^ { t } \left(\left\|\mathbf{X}_{s}^{M}-\mathbf{X}_{\lfloor s\rfloor_{T / M}}^{M}\right\|_{\mathcal{L}^{2 p}(\mathbb{P} ; V)}+\left\|\mathbf{X}_{\lfloor s\rfloor_{T / M}}^{M}-\overline{\mathcal{X}}_{\lfloor s\rfloor_{T / M}}^{M}\right\|_{\mathcal{L}^{2 p(\mathbb{P} ; V)}}+\left\|O_{s}-O_{\lfloor s\rfloor_{T / M}}\right\|_{\mathcal{L}^{2 p}(\mathbb{P} ; V)}\right.\right. \\
& \left.\left.+\left\|O_{\lfloor s\rfloor_{T / M}}-\mathcal{O}_{\lfloor s\rfloor_{T / M}}^{M}\right\|_{\mathcal{L}^{2 p(\mathbb{P} ; V)}}\right)^{2} d s\right]^{\frac{1}{2}} .
\end{aligned}
$$

Proof of Lemma 4.2. Note that Lemma 3.6 and Hölder's inequality imply

$$
\begin{aligned}
& \left\|\bar{X}_{t}-\mathbf{X}_{t}^{M}\right\|_{\mathcal{L}^{p}(\mathbb{P} ; H)} \\
& \leq \frac{e^{\kappa C T / 2} \sqrt{2^{[\varphi-1]^{+}} L}}{\sqrt{C(\kappa-2)}}\left[\int_{0}^{t} \|\left(1+\left\|\mathbf{X}_{s}^{M}\right\|_{V}^{\varphi}+\left\|O_{s}\right\|_{V}^{\varphi}+\left\|\mathcal{X}_{\lfloor s\rfloor_{T / M}}^{M}\right\|_{V}^{\varphi}\right)\left(\left\|\mathbf{X}_{s}^{M}-\mathbf{X}_{\lfloor s\rfloor_{T / M}}^{M}\right\|_{V}\right.\right. \\
& \left.\left.+\left\|\mathbf{X}_{\lfloor s\rfloor_{T / M}}^{M}-\overline{\mathcal{X}}_{\lfloor s\rfloor_{T / M}}^{M}\right\|_{V}+\left\|O_{s}-O_{\lfloor s\rfloor_{T / M}}\right\|_{V}+\left\|O_{\lfloor s\rfloor_{T / M}}-\mathcal{O}_{\lfloor s\rfloor_{T / M}}^{M}\right\|_{V}\right)^{2} \|_{\mathcal{L}^{p / 2}(\mathbb{P} ; \mathbb{R})} d s\right]^{\frac{1}{2}} \\
& \leq \frac{e^{\kappa C T / 2} \sqrt{2^{[\varphi-1]^{+}} L}}{\sqrt{C(\kappa-2)}}\left[\int_{0}^{t}\|1+\| \mathbf{X}_{s}^{M}\left\|_{V}^{\varphi}+\right\| O_{s}\left\|_{V}^{\varphi}+\right\| \mathcal{X}_{\lfloor s\rfloor_{T / M}^{M}}^{M}\left\|_{V}^{\varphi}\right\|_{\mathcal{L}^{p}(\mathbb{P} ; \mathbb{R})}\|\| \mathbf{X}_{s}^{M}-\mathbf{X}_{\lfloor s\rfloor_{T / M}}^{M} \|_{V}\right. \\
& \left.+\left\|\mathbf{X}_{\lfloor s\rfloor_{T / M}}^{M}-\overline{\mathcal{X}}_{\lfloor s\rfloor_{T / M}^{M}}^{M}\right\|_{V}+\left\|O_{s}-O_{\lfloor s\rfloor_{T / M}}\right\|_{V}+\left\|O_{\lfloor s\rfloor_{T / M}}-\mathcal{O}_{\lfloor s\rfloor_{T / M}}^{M}\right\|_{V} \|_{\mathcal{L}^{2 p}(\mathbb{P} ; \mathbb{R})}^{2} d s\right]^{\frac{1}{2}} .
\end{aligned}
$$

This shows

$$
\begin{aligned}
& \left\|\bar{X}_{t}-\mathbf{X}_{t}^{M}\right\|_{\mathcal{L}^{p}(\mathbb{P} ; H)} \\
& \leq \frac{e^{\kappa C T / 2 \sqrt{2[\varphi-1]^{+}} L}}{\sqrt{C(\kappa-2)}}\left[\int_{0}^{t}\left(1+\left\|\mathbf{X}_{s}^{M}\right\|_{\mathcal{L}^{p \varphi(\mathbb{P} ; V)}}^{\varphi}+\left\|O_{s}\right\|_{\mathcal{L}^{p \varphi(\mathbb{P} ; V)}}^{\varphi}+\left\|\mathcal{X}_{\lfloor s\rfloor_{T / M}}^{M}\right\|_{\mathcal{L}^{p \varphi}(\mathbb{P} ; V)}^{\varphi}\right)\right. \\
& \quad \cdot\left(\left\|\mathbf{X}_{s}^{M}-\mathbf{X}_{\lfloor s\rfloor_{T / M}}^{M}\right\|_{\mathcal{L}^{2 p}(\mathbb{P} ; V)}+\left\|\mathbf{X}_{\lfloor s\rfloor_{T / M}}^{M}-\overline{\mathcal{X}}_{\lfloor s\rfloor_{T / M}}^{M}\right\|_{\mathcal{L}^{2 p}(\mathbb{P} ; V)}+\left\|O_{s}-O_{\lfloor s\rfloor_{T / M}}\right\|_{\mathcal{L}^{2 p}(\mathbb{P} ; V)}\right. \\
& \left.\left.\quad+\left\|O_{\lfloor s\rfloor_{T / M}}-\mathcal{O}_{\lfloor s\rfloor_{T / M}}^{M}\right\|_{\mathcal{L}^{2 p}(\mathbb{P} ; V)}\right)^{2} d s\right]^{\frac{1}{2}} .
\end{aligned}
$$

The fact that $\forall x_{1}, x_{2}, x_{3}, x_{4} \in[0, \infty): \sqrt{x_{1}+x_{2}+x_{3}+x_{4}} \leq \sqrt{x_{1}}+\sqrt{x_{2}}+\sqrt{x_{3}}+\sqrt{x_{4}}$ completes the proof of Lemma 4.2. 


\subsection{Analysis of the error between the exact solution and the numerical approximation}

Lemma 4.3 (Strong temporal regularity of the semilinear integrated version of the numerical approximation). Assume the setting in Section 4.1 and let $p \in[1, \infty), \varrho \in[0,1-\rho), t_{1}, t_{2} \in[0, T]$, $M \in \mathbb{N}$ with $t_{1}<t_{2}$. Then

$$
\begin{aligned}
& \left\|\mathbf{X}_{t_{2}}^{M}-\mathbf{X}_{t_{1}}^{M}\right\|_{\mathcal{L}^{p}(\mathbb{P} ; V)} \leq\left(t_{2}-t_{1}\right)^{\varrho} T^{(1-\rho-\varrho)}\left[\sup _{s \in(0, T)} s^{\rho}\left\|e^{s A}\right\|_{L(H, V)}\right]\left[\sup _{s \in[0, T)}\left\|F\left(\mathcal{X}_{s}^{M}\right)\right\|_{\mathcal{L}^{p}(\mathbb{P} ; H)}\right] \\
& \cdot\left(\frac{1}{(1-\rho)}+\frac{2^{(\rho+\varrho)}}{(1-\rho-\varrho)}\left[\sup _{s \in(0, T)}\left\|(-s A)^{\varrho} e^{s A}\right\|_{L(H)}\right]\left[\sup _{s \in(0, T)}\left\|(-s A)^{-\varrho}\left(e^{s A}-\operatorname{Id}_{H}\right)\right\|_{L(H)}\right]\right) .
\end{aligned}
$$

Proof of Lemma 4.3. Note that Lemma 3.4 implies

$$
\begin{gathered}
\left\|\mathbf{X}_{t_{2}}^{M}-\mathbf{X}_{t_{1}}^{M}\right\|_{\mathcal{L}^{p}(\mathbb{P} ; V)} \leq\left[\sup _{s \in(0, T)} s^{\rho}\left\|e^{s A}\right\|_{L(H, V)}\right] \int_{t_{1}}^{t_{2}}\left(t_{2}-s\right)^{-\rho}\left\|F\left(\mathcal{X}_{\lfloor s\rfloor_{T / M}}^{M}\right)\right\|_{\mathcal{L}^{p}(\mathbb{P} ; H)} d s \\
+2^{(\rho+\varrho)}\left(t_{2}-t_{1}\right)^{\varrho}\left[\sup _{s \in(0, T)} s^{\rho}\left\|e^{s A}\right\|_{L(H, V)}\right]\left[\sup _{s \in(0, T)}\left\|(-s A)^{\varrho} e^{s A}\right\|_{L(H)}\right] \\
\quad \cdot\left[\sup _{s \in(0, T)}\left\|(-s A)^{-\varrho}\left(e^{s A}-\operatorname{Id}_{H}\right)\right\|_{L(H)}\right] \int_{0}^{t_{1}}\left(t_{1}-s\right)^{-(\rho+\varrho)}\left\|F\left(\mathcal{X}_{\lfloor s\rfloor_{T / M}^{M}}\right)\right\|_{\mathcal{L}^{p}(\mathbb{P} ; H)} d s .
\end{gathered}
$$

Hence, we obtain

$$
\begin{aligned}
& \left\|\mathbf{X}_{t_{2}}^{M}-\mathbf{X}_{t_{1}}^{M}\right\|_{\mathcal{L}^{p}(\mathbb{P} ; V)} \leq \frac{\left(t_{2}-t_{1}\right)^{(1-\rho)}}{(1-\rho)}\left[\sup _{s \in(0, T)} s^{\rho}\left\|e^{s A}\right\|_{L(H, V)}\right]\left[\sup _{s \in[0, T)}\left\|F\left(\mathcal{X}_{s}^{M}\right)\right\|_{\mathcal{L}^{p}(\mathbb{P} ; H)}\right] \\
& +\frac{2^{(\rho+\varrho)}\left(t_{2}-t_{1}\right)^{\varrho}\left|t_{1}\right|^{(1-\rho-\varrho)}}{(1-\rho-\varrho)}\left[\sup _{s \in(0, T)} s^{\rho}\left\|e^{s A}\right\|_{L(H, V)}\right]\left[\sup _{s \in(0, T)}\left\|(-s A)^{\varrho} e^{s A}\right\|_{L(H)}\right] \\
& \quad \cdot\left[\sup _{s \in(0, T)}\left\|(-s A)^{-\varrho}\left(e^{s A}-\operatorname{Id}_{H}\right)\right\|_{L(H)}\right]\left[\sup _{s \in[0, T)}\left\|F\left(\mathcal{X}_{s}^{M}\right)\right\|_{\mathcal{L}^{p}(\mathbb{P} ; H)}\right] .
\end{aligned}
$$

This completes the proof Lemma 4.3.

Proposition 4.4. Assume the setting in Section 4.1, let $\alpha \in(0, \infty), \theta \in(0,1 / 2), \varrho \in[0,1-\rho)$, $p \in[\max \{2,1 / \varphi\}, \infty)$, and assume that

$$
\begin{aligned}
& \sup _{M \in \mathbb{N}} \sup _{t \in[0, T]}\left\|\mathcal{X}_{t}^{M}\right\|_{\mathcal{L}^{p \max \{1, \varphi\}(\mathbb{P} ; V)}}+\sup _{M \in \mathbb{N}} \sup _{t \in[0, T)}\left\|\left|\mathcal{V}\left(\mathcal{X}_{t}^{M}\right)\right|^{2 \alpha}+\right\| F\left(\mathcal{X}_{t}^{M}\right)\left\|_{H}^{2}\right\|_{\mathcal{L}^{p \max \{2, \varphi\}(\mathbb{P} ; \mathbb{R})}} \\
& +\sup _{M \in \mathbb{N}} \sup _{t \in(0, T]} M^{\varrho} \int_{0}^{t}\left\|e^{(t-s) A}-\mathcal{S}_{\lfloor s\rfloor_{T / M}, t}^{M}\right\|_{L(H, V)} d s+\sup _{t \in[0, T)}\left\|O_{t}\right\|_{\mathcal{L}^{p \max \{2, \varphi\}(\mathbb{P} ; V)}} \\
& +\sup _{M \in \mathbb{N}} \sup _{t \in[0, T]} M^{\theta}\left[\left\|O_{t}-\mathcal{O}_{t}^{M}\right\|_{\mathcal{L}^{p \max \{2, \varphi\}(\mathbb{P} ; V)}}+\left|\lfloor t\rfloor_{T / M}\right|^{\theta}\left\|O_{t}-O_{\lfloor t\rfloor_{T / M}}\right\|_{\mathcal{L}^{2 p(\mathbb{P} ; V)}}\right]<\infty .
\end{aligned}
$$

Then $\sup _{M \in \mathbb{N}} \sup _{t \in[0, T]}\left[\left\|X_{t}\right\|_{\mathcal{L}^{p}(\mathbb{P} ; H)}+M^{\min \{\alpha, \varrho, \theta\}}\left\|X_{t}-\mathcal{X}_{t}^{M}\right\|_{\mathcal{L}^{p}(\mathbb{P} ; H)}\right]<\infty$. 
Proof of Proposition 4.4. First of all, observe that the triangle inequality and (74) implies

$$
\sup _{M \in \mathbb{N}} \sup _{t \in[0, T)}\left\|\mathcal{O}_{t}^{M}\right\|_{\mathcal{L}^{p \max \{2, \varphi\}(\mathbb{P} ; V)}} \leq \sup _{M \in \mathbb{N}} \sup _{t \in[0, T)}\|\| \mathcal{O}_{t}^{M}-O_{t}\left\|_{V}+\right\| O_{t}\left\|_{V}\right\|_{\mathcal{L}^{p \max \{2, \varphi\}(\mathbb{P} ; \mathbb{R})}}<\infty .
$$

This, the triangle inequality, and (74) assure that

$$
\sup _{M \in \mathbb{N}} \sup _{t \in[0, T)}\left\|\overline{\mathcal{X}}_{t}^{M}\right\|_{\mathcal{L}^{p \varphi}(\mathbb{P} ; V)} \leq \sup _{M \in \mathbb{N}} \sup _{t \in[0, T)}\left[\left\|\mathcal{X}_{t}^{M}\right\|_{\mathcal{L}^{p \max \{1, \varphi\}(\mathbb{P} ; V)}}+\left\|\mathcal{O}_{t}^{M}\right\|_{\mathcal{L}^{p \max \{2, \varphi\}(\mathbb{P} ; V)}}\right]<\infty
$$

Next note that Lemma 4.1, (174), and the assumption that $\sup _{t \in(0, T)} t^{\rho}\left\|e^{t A}\right\|_{L(H, V)}<\infty$ prove that

$$
\begin{aligned}
& \sup _{M \in \mathbb{N}} \sup _{t \in[0, T]} M^{\min \{\alpha, \varrho\}}\left\|\mathbf{X}_{t}^{M}-\overline{\mathcal{X}}_{t}^{M}\right\|_{\mathcal{L}^{p \max \{2, \varphi\}(\mathbb{P} ; V)}}=\sup _{M \in \mathbb{N}} \sup _{t \in(0, T]} M^{\min \{\alpha, \varrho\}}\left\|\mathbf{X}_{t}^{M}-\overline{\mathcal{X}}_{t}^{M}\right\|_{\mathcal{L}^{p \max \{2, \varphi\}(\mathbb{P} ; V)}} \\
& \leq \sup _{M \in \mathbb{N}} \sup _{t \in(0, T]}\left\{\left(M^{\varrho} \int_{0}^{t}\left\|e^{(t-s) A}-\mathcal{S}_{\lfloor s\rfloor_{T / M}, t}^{M}\right\|_{L(H, V)} d s\right)\left[\sup _{s \in[0, T)}\left\|F\left(\mathcal{X}_{s}^{M}\right)\right\|_{\mathcal{L}^{p \max \{2, \varphi\}(\mathbb{P} ; H)}}\right]\right\} \\
& +\frac{T^{(1+\alpha-\rho)}}{(1-\rho)}\left[\sup _{s \in(0, T)} s^{\rho}\left\|e^{s A}\right\|_{L(H, V)}\right]\left[\sup _{M \in \mathbb{N}} \sup _{s \in[0, T)}\left\|\mathcal{V}\left(\mathcal{X}_{s}^{M}\right)\right\|_{\mathcal{L}^{2 \alpha p \max \{2, \varphi\}(\mathbb{P} ; \mathbb{R})}}^{\alpha}\left\|F\left(\mathcal{X}_{s}^{M}\right)\right\|_{\mathcal{L}^{2 p \max \{2, \varphi\}(\mathbb{P} ; H)}}\right] \\
& <\infty \text {. }
\end{aligned}
$$

Combining this, the triangle inequality, and (76) ensures

$$
\sup _{M \in \mathbb{N}} \sup _{t \in[0, T)}\left\|\mathbf{X}_{t}^{M}\right\|_{\mathcal{L}^{p \varphi}(\mathbb{P} ; V)} \leq \sup _{M \in \mathbb{N}} \sup _{t \in[0, T)}\left[\left\|\mathbf{X}_{t}^{M}-\overline{\mathcal{X}}_{t}^{M}\right\|_{\mathcal{L}^{p \max \{2, \varphi\}(\mathbb{P} ; V)}}+\left\|\overline{\mathcal{X}}_{t}^{M}\right\|_{\mathcal{L}^{p \varphi}(\mathbb{P} ; V)}\right]<\infty
$$

Furthermore, observe that the fact that $A: D(A) \subseteq H \rightarrow H$ is a generator of an analytic semigroup with $\operatorname{spectrum}(A) \subseteq\{z \in \mathcal{C}: \operatorname{Re}(z)<0\}$ implies that $\sup _{s \in(0, T)}\left\|(-s A)^{-\varrho}\left(e^{s A}-\operatorname{Id}_{H}\right)\right\|_{L(H)}+$ $\sup _{s \in(0, T)}\left\|(-s A) \varrho e^{s A}\right\|_{L(H)}<\infty$. This, the assumption that $\sup _{t \in(0, T)} t^{\rho}\left\|e^{t A}\right\|_{L(H, V)}<\infty$, (174), and Lemma 4.3 show

$$
\begin{aligned}
& \sup _{M \in \mathbb{N}} \sup _{t \in[0, T]} M^{\varrho}\left\|\mathbf{X}_{t}^{M}-\mathbf{X}_{\lfloor t\rfloor_{T / M}^{M}}^{M}\right\|_{\mathcal{L}^{2 p}(\mathbb{P} ; V)} \\
& \leq\left(\frac{1}{(1-\rho)}+\frac{2^{(\rho+\varrho)}}{(1-\rho-\varrho)}\left[\sup _{s \in(0, T)}\left\|(-s A)^{\varrho} e^{s A}\right\|_{L(H)}\right]\left[\sup _{s \in(0, T)}\left\|(-s A)^{-\varrho}\left(e^{s A}-\operatorname{Id}_{H}\right)\right\|_{L(H)}\right]\right) \\
& \cdot T^{(1-\rho)}\left[\sup _{s \in(0, T)} s^{\rho}\left\|e^{s A}\right\|_{L(H, V)}\right]\left[\sup _{M \in \mathbb{N}} \sup _{s \in[0, T)}\left\|F\left(\mathcal{X}_{s}^{M}\right)\right\|_{\mathcal{L}^{2 p}(\mathbb{P} ; H)}\right]<\infty .
\end{aligned}
$$

Moreover, note that Lemma 4.2, the triangle inequality, and the fact that $\forall x, y \in \mathbb{R}:(x+y)^{2} \leq$ 
$2\left(x^{2}+y^{2}\right)$ assure for all $\kappa \in(2, \infty)$ that

$$
\begin{aligned}
& \sup _{M \in \mathbb{N}} \sup _{t \in[0, T]} M^{\min \{\alpha, \varrho, \theta\}}\left\|\bar{X}_{t}-\mathbf{X}_{t}^{M}\right\|_{\mathcal{L}^{p}(\mathbb{P} ; H)} \\
& \leq \frac{e^{\kappa C T / 2} \sqrt{2^{[\varphi-1]^{+}} L}}{\sqrt{C(\kappa-2)}}\left[1+\sup _{M \in \mathbb{N}} \sup _{s \in(0, T)}\left\|\mathbf{X}_{s}^{M}\right\|_{\mathcal{L}^{p \varphi}(\mathbb{P} ; V)}^{\varphi / 2}+\sup _{s \in(0, T)}\left\|O_{s}\right\|_{\mathcal{L}^{p \varphi}(\mathbb{P} ; V)}^{\varphi / 2}+\sup _{M \in \mathbb{N}} \sup _{s \in[0, T)}\left\|\mathcal{X}_{s}^{M}\right\|_{\mathcal{L}^{p \varphi}(\mathbb{P} ; V)}^{\varphi / 2}\right] \\
& \cdot \sup _{M \in \mathbb{N}} M^{\min \{\alpha, \varrho, \theta\}}\left\{\left[\int_{0}^{\frac{T}{M}}\left(\left\|O_{s}\right\|_{\mathcal{L}^{2 p}(\mathbb{P} ; V)}+\left\|O_{\lfloor s\rfloor_{T / M}}\right\|_{\mathcal{L}^{2 p}(\mathbb{P} ; V)}\right)^{2} d s+\int_{\frac{T}{M}}^{T}\left\|O_{s}-O_{\lfloor s\rfloor_{T / M}}\right\|_{\mathcal{L}^{2 p}(\mathbb{P} ; V)}^{2} d s\right]^{\frac{1}{2}}\right. \\
& +\left[\int _ { 0 } ^ { T } \left(\left\|\mathbf{X}_{s}^{M}-\mathbf{X}_{\lfloor s\rfloor_{T / M}}^{M}\right\|\left\|_{\mathcal{L}^{2 p}(\mathbb{P} ; V)}+\right\| \mathbf{X}_{\lfloor s\rfloor_{T / M}}^{M}-\overline{\mathcal{X}}_{\lfloor s\rfloor_{T / M}^{M}}^{M} \|_{\mathcal{L}^{2 p}(\mathbb{P} ; V)}\right.\right. \\
& \left.\left.\left.+\left\|O_{\lfloor s\rfloor_{T / M}}-\mathcal{O}_{\lfloor s\rfloor_{T / M}}^{M}\right\|_{\mathcal{L}^{2 p(\mathbb{P} ; V)}}\right)^{2} d s\right]^{\frac{1}{2}}\right\} .
\end{aligned}
$$

The estimate

$$
\begin{aligned}
& \sup _{M \in \mathbb{N}}\left[\int_{\frac{T}{M}}^{T}\left|\lfloor s\rfloor_{T / M}\right|^{-2 \theta} d s\right]=\sup _{M \in \mathbb{N}}\left[\sum_{l=1}^{M-1} \int_{\frac{l T}{M}}^{\frac{(l+1) T}{M}} \frac{M^{2 \theta}}{(l T)^{2 \theta}} d s\right]=\sup _{M \in \mathbb{N}}\left[\frac{T^{(1-2 \theta)}}{M^{(1-2 \theta)}} \sum_{l=1}^{M-1} \frac{1}{l^{2 \theta}}\right] \\
& \leq \sup _{M \in \mathbb{N}}\left[\frac{T^{(1-2 \theta)}}{M^{(1-2 \theta)}}\left(1+\int_{1}^{M} \frac{1}{s^{2 \theta}} d s\right)\right]=\sup _{M \in \mathbb{N}}\left[\frac{T^{(1-2 \theta)}}{M^{(1-2 \theta)}}\left(1+\frac{\left(M^{(1-2 \theta)}-1\right)}{(1-\theta)}\right)\right] \leq \frac{T^{(1-2 \theta)}}{(1-\theta)},
\end{aligned}
$$

inequality (74), inequalities (77) -(79), and the fact that $\forall x, y \in[0, \infty): \sqrt{x+y} \leq \sqrt{x}+\sqrt{y}$ therefore imply that

$$
\begin{aligned}
& \sup _{M \in \mathbb{N}} \sup _{t \in[0, T]} M^{\min \{\alpha, \varrho, \theta\}}\left\|\bar{X}_{t}-\mathbf{X}_{t}^{M}\right\|_{\mathcal{L}^{p}(\mathbb{P} ; H)} \\
& \leq \frac{e^{\kappa C T / 2} \sqrt{2^{[\varphi-1]^{+}} L}}{\sqrt{C(\kappa-2)}}\left[1+\sup _{M \in \mathbb{N}} \sup _{s \in(0, T)}\left\|\mathbf{X}_{s}^{M}\right\|_{\mathcal{L}^{p \varphi}(\mathbb{P} ; V)}^{\varphi / 2}+\sup _{s \in(0, T)}\left\|O_{s}\right\|_{\mathcal{L}^{p \varphi}(\mathbb{P} ; V)}^{\varphi / 2}+\sup _{M \in \mathbb{N}} \sup _{s \in[0, T)}\left\|\mathcal{X}_{s}^{M}\right\|_{\mathcal{L}^{p \varphi}(\mathbb{P} ; V)}^{\varphi / 2}\right] \\
& \cdot \sqrt{(1+T)} \sup _{M \in \mathbb{N}}\left[2 \sup _{s \in[0, T)}\left\|O_{s}\right\|_{\mathcal{L}^{2 p}(\mathbb{P} ; V)}+\sup _{s \in(0, T)}\left|M\lfloor s\rfloor_{T / M}\right|^{\theta}\left\|O_{s}-O_{\lfloor s\rfloor_{T / M}}\right\|_{\mathcal{L}^{2 p}(\mathbb{P} ; V)}\left(\int_{\frac{T}{M}}^{T}\left|\lfloor s\rfloor_{T / M}\right|^{-2 \theta} d s\right)^{\frac{1}{2}}\right. \\
& \left.+\sup _{s \in[0, T]}\left(M^{\varrho}\left\|\mathbf{X}_{s}^{M}-\mathbf{X}_{\lfloor s\rfloor_{T / M}}^{M}\right\|_{\mathcal{L}^{2 p(\mathbb{P} ; V)}}+M^{\min \{\alpha, \varrho\}}\left\|\mathbf{X}_{s}^{M}-\overline{\mathcal{X}}_{s}^{M}\right\|_{\mathcal{L}^{2 p(\mathbb{P} ; V)}}+M^{\theta}\left\|O_{s}-\mathcal{O}_{s}^{M}\right\|_{\mathcal{L}^{2 p}(\mathbb{P} ; V)}\right)\right] \\
& <\infty .
\end{aligned}
$$

Next note that the triangle inequality, the assumption that $V \subseteq H$ continuously, (74), (777), and (82) 
prove

$$
\begin{aligned}
& \sup _{M \in \mathbb{N}} \sup _{t \in[0, T]} M^{\min \{\alpha, \varrho, \theta\}}\left\|X_{t}-\mathcal{X}_{t}^{M}\right\|_{\mathcal{L}^{p}(\mathbb{P} ; H)} \\
& \leq \sup _{M \in \mathbb{N}} \sup _{t \in[0, T]} M^{\min \{\alpha, \varrho, \theta\}}\left[\left\|\bar{X}_{t}-\overline{\mathcal{X}}_{t}^{M}\right\|_{\mathcal{L}^{p}(\mathbb{P} ; H)}+\left\|O_{t}-\mathcal{O}_{t}^{M}\right\|_{\mathcal{L}^{p}(\mathbb{P} ; H)}\right] \\
& \leq \sup _{M \in \mathbb{N}} \sup _{t \in[0, T]} M^{\min \{\alpha, \varrho, \theta\}}\left\|\bar{X}_{t}-\mathbf{X}_{t}^{M}\right\|_{\mathcal{L}^{p}(\mathbb{P} ; H)} \\
& \quad+\left[\sup _{v \in V \backslash\{0\}} \frac{\|v\|_{H}}{\|v\|_{V}}\right] \sup _{M \in \mathbb{N}} \sup _{t \in[0, T]} M^{\min \{\alpha, \varrho\}}\left\|\mathbf{X}_{t}^{M}-\overline{\mathcal{X}}_{t}^{M}\right\|_{\mathcal{L}^{p \max \{2, \varphi\}(\mathbb{P} ; V)}} \\
& \quad+\left[\sup _{v \in V \backslash\{0\}} \frac{\|v\|_{H}}{\|v\|_{V}}\right] \sup _{M \in \mathbb{N}} \sup _{t \in[0, T]} M^{\theta}\left\|O_{t}-\mathcal{O}_{t}^{M}\right\|_{\mathcal{L}^{p \max \{2, \varphi\}(\mathbb{P} ; V)}}<\infty .
\end{aligned}
$$

This, the triangle inequality, the assumption that $V \subseteq H$ continuously, and (74) assure that

$$
\sup _{t \in[0, T]}\left\|X_{t}\right\|_{\mathcal{L}^{p}(\mathbb{P} ; H)} \leq \sup _{M \in \mathbb{N}} \sup _{t \in[0, T]}\left[\left\|X_{t}-\mathcal{X}_{t}^{M}\right\|_{\mathcal{L}^{p}(\mathbb{P} ; H)}+\left[\sup _{v \in V \backslash\{0\}} \frac{\|v\|_{H}}{\|v\|_{V}}\right]\left\|\mathcal{X}_{t}^{M}\right\|_{\mathcal{L}^{p \max \{1, \varphi\}(\mathbb{P} ; V)}}\right]<\infty .
$$

The proof of Proposition 4.4 is thus completed.

\section{Main result}

\section{$5.1 \quad$ Setting}

Let $T, c, \varphi, \phi, \chi \in(0, \infty)$, let $\left(H,\langle\cdot, \cdot\rangle_{H},\|\cdot\|_{H}\right)$ be a separable $\mathbb{R}$-Hilbert space, let $A: D(A) \subseteq$ $H \rightarrow H$ be a generator of an analytic semigroup with $\operatorname{spectrum}(A) \subseteq\{z \in \mathbb{C}: \operatorname{Re}(z)<0\}$, let $\left(H_{r},\langle\cdot, \cdot\rangle_{H_{r}},\|\cdot\|_{H_{r}}\right), r \in \mathbb{R}$, be a family of interpolation spaces associated to $-A$ (cf., e.g., Definition 3.5.26 in [23]), let $\left(V,\|\cdot\|_{V}\right)$ be a separable $\mathbb{R}$-Banach space with $H_{1} \subseteq V \subseteq H$ densely and continuously, let $F \in \mathcal{C}(V, H), \Phi, \Psi \in \mathcal{M}(\mathcal{B}(V), \mathcal{B}([0, \infty))),\left(\mathcal{S}^{M}\right)_{M \in \mathbb{N}} \subseteq \mathcal{M}\left(\mathcal{B}\left(\left\{(s, t) \in[0, T]^{2}: s<\right.\right.\right.$ $t\}), \mathcal{B}(L(H, V)))$ satisfy for all $u, v, x, y \in V, M \in \mathbb{N}, r_{1}, r_{2}, r_{3} \in[0, T], s \in\left\{0, \frac{T}{M}, \frac{2 T}{M}, \ldots, \frac{(M-1) T}{M}\right\}$, $t \in\left(s, s+\frac{T}{M}\right]$ with $x-y \in H_{1}$ and $r_{1}<r_{2}<r_{3}$ that $\|F(u+v)\|_{H} \leq c\left(1+|\Phi(u)|^{\phi}+|\Phi(v)|^{\phi}\right)$, $\|F(x)-F(y)\|_{H}^{2} \leq c\|x-y\|_{V}^{2}\left(1+\|x\|_{V}^{\varphi}+\|y\|_{V}^{\varphi}\right),\langle x-y, A(x-y)+F(x)-F(y)\rangle_{H} \leq c\|x-y\|_{H}^{2}$, $\mathcal{S}_{r_{1}, r_{3}}^{M}=\mathcal{S}_{r_{2}, r_{3}}^{M} \mathcal{S}_{r_{1}, r_{2}}^{M}$, and

$$
\Phi\left(\mathcal{S}_{s, t}^{M}\left[u+(t-s) \mathbb{1}_{\left[0,(M / T)^{\chi}\right]}\left(\|u+v\|_{V}\right) F(u+v)\right]\right) \leq e^{c(t-s)}[\Phi(u)+(t-s) \Psi(v)],
$$

let $(\Omega, \mathcal{F}, \mathbb{P})$ be a probability space, let $O, X:[0, T] \times \Omega \rightarrow V$ be stochastic processes with continuous sample paths, let $\mathcal{O}^{M}, \mathcal{X}^{M}:[0, T] \times \Omega \rightarrow V, M \in \mathbb{N}$, be stochastic processes, and assume for all $t \in[0, T], M \in \mathbb{N}$ that $\mathbb{P}\left(\lim \sup _{r \searrow 0} \sup _{0 \leq s<u \leq T} \frac{s\left\|O_{u}-O_{s}\right\|_{V}}{(u-s)^{r}}<\infty\right)=\mathbb{P}\left(X_{t}=\int_{0}^{t} e^{(t-s) A} F\left(X_{s}\right) d s+\right.$ $\left.O_{t}\right)=\mathbb{P}\left(\mathcal{X}_{t}^{M}=\int_{0}^{t} \mathcal{S}_{\lfloor s\rfloor_{T / M}, t}^{M} \mathbb{1}_{\left\{\left\|\mathcal{X}_{\lfloor s\rfloor_{T / M}^{M}}^{M}\right\|_{V} \leq(M / T) \chi\right\}} F\left(\mathcal{X}_{\lfloor s\rfloor_{T / M}}^{M}\right) d s+\mathcal{O}_{t}^{M}\right)=1$. 


\subsection{A priori bounds}

Corollary 5.1. Assume the setting in Section 5.1, let $p \in[\max \{1,1 / \phi\}, \infty), \rho \in[0,1)$, and assume that $\sup _{M \in \mathbb{N}} \sup _{0 \leq s<t<T}(t-s)^{\rho}\left\|\mathcal{S}_{[s\rfloor_{T / M}, t}^{M}\right\|_{L(H, V)}+\sup _{M \in \mathbb{N}} \sup _{t \in[0, T)}\left\|\Phi\left(\mathcal{O}_{t}^{M}\right)+\Psi\left(\mathcal{O}_{t}^{M}\right)\right\|_{\mathcal{L}^{p \phi}(\mathbb{P} ; \mathbb{R})}$ $+\sup _{M \in \mathbb{N}} \sup _{t \in[0, T]}\left\|\mathcal{O}_{t}^{M}\right\|_{\mathcal{L}^{p}(\mathbb{P} ; V)}<\infty$. Then $\sup _{M \in \mathbb{N}} \sup _{t \in[0, T]}\left\|\mathcal{X}_{t}^{M}\right\|_{\mathcal{L}^{p}(\mathbb{P} ; V)}<\infty$.

Proof of Corollary [5.1. Throughout this proof let $\tilde{\mathcal{X}}^{M}:[0, T] \times \Omega \rightarrow V, M \in \mathbb{N}$, be the stochastic processes which satisfy for all $t \in[0, T], M \in \mathbb{N}$ that

$$
\tilde{\mathcal{X}}_{t}^{M}=\int_{0}^{t} \mathcal{S}_{\lfloor s\rfloor_{T / M}, t}^{M} \mathbb{1}_{\left\{\left\|\tilde{\mathcal{X}}_{\lfloor s\rfloor_{T / M}^{M}}^{M}\right\|_{V} \leq(M / T)^{\chi}\right\}} F\left(\tilde{\mathcal{X}}_{\lfloor s\rfloor_{T / M}^{M}}^{M}\right) d s+\mathcal{O}_{t}^{M}
$$

Next note that the triangle inequality, the fact that $\forall t \in[0, T], M \in \mathbb{N}: \mathbb{P}\left(\mathcal{X}_{t}^{M}=\tilde{\mathcal{X}}_{t}^{M}\right)=1$, and Corollary 2.4 (with $Y_{t}=\tilde{\mathcal{X}}_{t}^{M}-\mathcal{O}_{t}^{M}$ for $t \in(0, T], M \in \mathbb{N}$ in the notation of Corollary 2.4) prove that

$$
\begin{aligned}
& \sup _{M \in \mathbb{N}} \sup _{t \in[0, T]}\left\|\mathcal{X}_{t}^{M}\right\|_{\mathcal{L}^{p}(\mathbb{P} ; V)}=\sup _{M \in \mathbb{N}} \sup _{t \in[0, T]}\left\|\tilde{\mathcal{X}}_{t}^{M}\right\|_{\mathcal{L}^{p}(\mathbb{P} ; V)} \\
& \leq \sup _{M \in \mathbb{N}} \sup _{t \in[0, T]}\left\|\mathcal{O}_{t}^{M}\right\|_{\mathcal{L}^{p}(\mathbb{P} ; V)}+\sup _{M \in \mathbb{N}} \sup _{t \in(0, T]}\left\|\tilde{\mathcal{X}}_{t}^{M}-\mathcal{O}_{t}^{M}\right\|_{\mathcal{L}^{p}(\mathbb{P} ; V)} \\
& \leq \sup _{M \in \mathbb{N}} \sup _{t \in[0, T]}\left\|\mathcal{O}_{t}^{M}\right\|_{\mathcal{L}^{p}(\mathbb{P} ; V)}+\frac{c T^{(1-\rho)}}{(1-\rho)}\left[\sup _{M \in \mathbb{N}} \sup _{0 \leq s<t<T}(t-s)^{\rho}\left\|\mathcal{S}_{\lfloor s\rfloor_{T / M}, t}\right\|_{L(H, V)}\right] \\
& \cdot\left(1+\sup _{M \in \mathbb{N}} \sup _{t \in[0, T)}\left\|\Phi\left(\mathcal{O}_{t}^{M}\right)\right\|_{\mathcal{L}^{p \phi}(\mathbb{P} ; \mathbb{R})}^{\phi}+2^{[\phi-1]^{+}} e^{c \phi T}\left[|\Phi(0)|^{\phi}+T^{\phi} \sup _{M \in \mathbb{N}} \sup _{t \in[0, T)}\left\|\Psi\left(\mathcal{O}_{t}^{M}\right)\right\|_{\mathcal{L}^{p \phi}(\mathbb{P} ; \mathbb{R})}^{\phi}\right]\right)<\infty .
\end{aligned}
$$

The proof of Corollary 5.1 is thus completed.

Corollary 5.2. Assume the setting in Section [5.1, let $p \in[\max \{1,1 / \phi\}, \infty)$, and assume that $\sup _{M \in \mathbb{N}} \sup _{t \in[0, T)}\left\|\Phi\left(\mathcal{O}_{t}^{M}\right)+\Psi\left(\mathcal{O}_{t}^{M}\right)\right\|_{\mathcal{L}^{p \phi}(\mathbb{P} ; \mathbb{R})}<\infty$. Then $\sup _{M \in \mathbb{N}} \sup _{t \in[0, T)}\left\|F\left(\mathcal{X}_{t}^{M}\right)\right\|_{\mathcal{L}^{p}(\mathbb{P} ; H)}<\infty$.

Proof of Corollary 5.2. Throughout this proof let $\tilde{\mathcal{X}}^{M}:[0, T] \times \Omega \rightarrow V, M \in \mathbb{N}$, be the stochastic processes which satisfy for all $t \in[0, T], M \in \mathbb{N}$ that

$$
\tilde{\mathcal{X}}_{t}^{M}=\int_{0}^{t} \mathcal{S}_{\lfloor s\rfloor_{T / M}, t}^{M} \mathbb{1}_{\left\{\left\|\tilde{\mathcal{X}}_{\lfloor s\rfloor_{T / M}^{M}}^{M}\right\|_{V} \leq(M / T)^{\chi}\right\}} F\left(\tilde{\mathcal{X}}_{\lfloor s\rfloor_{T / M}^{M}}^{M}\right) d s+\mathcal{O}_{t}^{M}
$$

In the next step observe that Lemma 2.2 (with $Y_{t}=\tilde{\mathcal{X}}_{t}^{M}-\mathcal{O}_{t}^{M}$ for $t \in[0, T], M \in \mathbb{N}$ in the notation of Lemma 2.2) ensures that for all $t \in[0, T], M \in \mathbb{N}$ it holds that

$$
\left\|F\left(\tilde{\mathcal{X}}_{t}^{M}\right)\right\|_{H} \leq c\left(1+2^{[\phi-1]^{+}} e^{c \phi t}\left[|\Phi(0)|^{\phi}+\left|\int_{0}^{t} \Psi\left(\mathcal{O}_{\lfloor s\rfloor_{T / M}}^{M}\right) d s\right|^{\phi}\right]+\left|\Phi\left(\mathcal{O}_{t}^{M}\right)\right|^{\phi}\right) .
$$


This and the fact that $\forall t \in[0, T], M \in \mathbb{N}: \mathbb{P}\left(\mathcal{X}_{t}^{M}=\tilde{\mathcal{X}}_{t}^{M}\right)=1$ imply that

$$
\begin{aligned}
& \sup _{M \in \mathbb{N}} \sup _{t \in[0, T)}\left\|F\left(\mathcal{X}_{t}^{M}\right)\right\|_{\mathcal{L}^{p}(\mathbb{P} ; H)}=\sup _{M \in \mathbb{N}} \sup _{t \in[0, T)}\left\|F\left(\tilde{\mathcal{X}}_{t}^{M}\right)\right\|_{\mathcal{L}^{p}(\mathbb{P} ; H)} \\
& \leq \sup _{M \in \mathbb{N}} \sup _{t \in[0, T)}\left\{c\left(1+2^{[\phi-1]^{+}} e^{c \phi t}\left[|\Phi(0)|^{\phi}+t^{\phi} \sup _{s \in[0, t)}\left\|\Psi\left(\mathcal{O}_{\lfloor s\rfloor_{T / M}^{M}}^{M}\right)\right\|_{\mathcal{L}^{p \phi}(\mathbb{P} ; \mathbb{R})}^{\phi}\right]+\left\|\Phi\left(\mathcal{O}_{t}^{M}\right)\right\|_{\mathcal{L}^{p \phi}(\mathbb{P} ; \mathbb{R})}^{\phi}\right)\right\} \\
& \leq c\left(1+2^{[\phi-1]^{+}} e^{c \phi T}\left[|\Phi(0)|^{\phi}+T^{\phi} \sup _{M \in \mathbb{N}} \sup _{t \in[0, T)}\left\|\Psi\left(\mathcal{O}_{\lfloor t]_{T / M}^{M}}^{M}\right)\right\|_{\mathcal{L}^{p \phi}(\mathbb{P} ; \mathbb{R})}^{\phi}\right]+\sup _{M \in \mathbb{N}} \sup _{t \in[0, T)}\left\|\Phi\left(\mathcal{O}_{t}^{M}\right)\right\|_{\mathcal{L}^{p \phi}(\mathbb{P} ; \mathbb{R})}^{\phi}\right) \\
& <\infty .
\end{aligned}
$$

The proof of Corollary 5.2 is thus completed.

\subsection{Main result}

Theorem 5.3. Assume the setting in Section 5.1 , let $\vartheta \in(0, \infty), \theta \in(0,1 / 2), \rho \in[0,1), \varrho \in$ $[0,1-\rho), p \in\left[\max \left\{2, \frac{1}{\varphi}, \frac{1}{2 \phi} \min \left\{1, \frac{2}{\varphi}, \frac{1}{2 \vartheta}, \frac{1}{\vartheta \varphi}\right\}\right\}, \infty\right)$, and assume that

$$
\begin{aligned}
& \sup _{M \in \mathbb{N}} \sup _{0 \leq s<t \leq T}\left[t^{\rho}\left\|e^{t A}\right\|_{L(H, V)}+(t-s)^{\rho}\left\|\mathcal{S}_{\lfloor s\rfloor_{T / M}, t}^{M}\right\|_{L(H, V)}+M^{\varrho} \int_{0}^{t}\left\|e^{(t-u) A}-\mathcal{S}_{\lfloor u\rfloor_{T / M}, t}^{M}\right\|_{L(H, V)} d u\right] \\
& +\sup _{M \in \mathbb{N}} \sup _{t \in[0, T]}\left[\left\|\Phi\left(\mathcal{O}_{t}^{M}\right)+\Psi\left(\mathcal{O}_{t}^{M}\right)\right\|_{\mathcal{L}^{2 p \phi \max \{2, \varphi, 2 \vartheta, \vartheta \varphi\}(\mathbb{P} ; \mathbb{R})}}+\left|M\lfloor t\rfloor_{T / M}\right|^{\theta}\left\|O_{t}-O_{\lfloor t\rfloor_{T / M}}\right\|_{\mathcal{L}^{2 p(\mathbb{P} ; V)}}\right] \\
& +\sup _{M \in \mathbb{N}} \sup _{t \in[0, T]}\|\| O_{t}\left\|_{V}+M^{\theta}\right\| O_{t}-\mathcal{O}_{t}^{M}\left\|_{V}\right\|_{\mathcal{L}^{p \max \{2, \varphi, 4 \vartheta, 2 \vartheta \varphi\}(\mathbb{P} ; \mathbb{R})}}<\infty .
\end{aligned}
$$

Then

$$
\sup _{M \in \mathbb{N}} \sup _{t \in[0, T]}\left[\left\|\mathcal{X}_{t}^{M}\right\|_{\mathcal{L}^{p \max \{2, \varphi, 4 \vartheta, 2 \vartheta \varphi\}(\mathbb{P} ; V)}}+\left\|X_{t}\right\|_{\mathcal{L}^{p}(\mathbb{P} ; H)}+M^{\min \{\vartheta \chi, \varrho, \theta\}}\left\|X_{t}-\mathcal{X}_{t}^{M}\right\|_{\mathcal{L}^{p}(\mathbb{P} ; H)}\right]<\infty .
$$

Proof of Theorem 5.3. Throughout this proof assume w.l.o.g. that $H \neq\{0\}$, let $\tilde{\mathcal{X}}^{M}:[0, T] \times \Omega \rightarrow$ $V, M \in \mathbb{N}$, be the stochastic processes which satisfy for all $t \in[0, T], M \in \mathbb{N}$ that $\tilde{\mathcal{X}}_{t}^{M}=$ $\int_{0}^{t} \mathcal{S}_{\lfloor s\rfloor_{T / M}, t}^{M} \mathbb{1}_{\left\{\left\|\tilde{\mathcal{X}}_{\lfloor s\rfloor_{T / M}^{M}}\right\|_{V} \leq(M / T) \chi\right\}} F\left(\tilde{\mathcal{X}}_{\lfloor s\rfloor_{T / M}^{M}}^{M}\right) d s+\mathcal{O}_{t}^{M}$, let $\tilde{\Omega} \subseteq \Omega$ be the set given by $\tilde{\Omega}=\{\omega \in \Omega:(\forall t \in$ $\left.\left.[0, T]: X_{t}(\omega)=\int_{0}^{t} e^{(t-s) A} F\left(X_{s}(\omega)\right) d s+O_{t}(\omega)\right)\right\} \cap\left\{\omega \in \Omega: \lim \sup _{r \searrow 0} \sup _{0 \leq s<u \leq T} \frac{s\left\|O_{u}(\omega)-O_{s}(\omega)\right\|_{V}}{(u-s)^{r}}<\right.$ $\infty\}$, let $\tilde{O}, \tilde{X}:[0, T] \times \Omega \rightarrow V$ be the stochastic processes which satisfy for all $t \in[0, T], \omega \in \tilde{\Omega}$ that $\tilde{O}_{t}(\omega)=O_{t}(\omega), \tilde{X}_{t}(\omega)=X_{t}(\omega)$ and which satisfy for all $t \in[0, T], \omega \in \Omega \backslash \tilde{\Omega}$ that $\tilde{O}_{t}(\omega)=$ $-\int_{0}^{t} e^{(t-s) A} F(0) d s, \tilde{X}_{t}(\omega)=0$, let $\kappa \in(0,1-\rho)$ be a real number, and let $K \in[0, \infty)$ be the real number with the property that $K=\|F(0)\|_{H}+\sup _{t \in(0, T]}\left[t^{\rho}\left\|e^{t A}\right\|_{L(H, V)}+\left\|(-t A)^{\kappa} e^{t A}\right\|_{L(H)}+\right.$ $\left.\left\|(-t A)^{-\kappa}\left(e^{t A}-\mathrm{Id}_{H}\right)\right\|_{L(H)}\right]$. The assumption that $\sup _{t \in(0, T]} t^{\rho}\left\|e^{t A}\right\|_{L(H, V)}<\infty$ and the assumption that $A: D(A) \subseteq H \rightarrow H$ is a generator of an analytic semigroup with $\operatorname{spectrum}(A) \subseteq\{z \in$ $\mathbb{C}: \operatorname{Re}(z)<0\}$ ensure that such a real number $K$ does indeed exist. Next note that for all $s, t \in[0, T]$, 
$\omega \in \Omega \backslash \tilde{\Omega}$ with $s<t$ it holds that

$$
\begin{aligned}
&\left\|\tilde{O}_{t}(\omega)-\tilde{O}_{s}(\omega)\right\|_{V}=\left\|\int_{0}^{t} e^{(t-u) A} F(0) d u-\int_{0}^{s} e^{(s-u) A} F(0) d u\right\|_{V} \\
& \leq\left\|\int_{s}^{t} e^{(t-u) A} F(0) d u\right\|_{V}+\left\|\int_{0}^{s}\left(e^{(t-u) A}-e^{(s-u) A}\right) F(0) d u\right\|_{V} \\
& \leq \int_{s}^{t}\left\|e^{(t-u) A}\right\|_{L(H, V)}\|F(0)\|_{H} d u+\int_{0}^{s}\left\|e^{\frac{1}{2}(s-u) A}\right\|_{L(H, V)}\left\|e^{\frac{1}{2}(s-u) A}\left(e^{(t-s)}-\operatorname{Id}_{H}\right) F(0)\right\|_{H} d u \\
& \leq\left[\sup _{u \in(0, t-s)} u^{\rho}\left\|e^{u A}\right\|_{L(H, V)}\right] \int_{s}^{t}(t-u)^{-\rho}\|F(0)\|_{H} d u+2^{\rho}\left[\sup _{u \in(0, s)} u^{\rho}\left\|e^{u A}\right\|_{L(H, V)}\right] \\
& \cdot \int_{0}^{s}(s-u)^{-\rho}\left\|(-A)^{\kappa} e^{\frac{1}{2}(s-u) A}\right\|_{L(H)}\left\|(-A)^{-\kappa}\left(e^{(t-s) A}-\operatorname{Id}_{H}\right)\right\|_{L(H)}\|F(0)\|_{H} d u .
\end{aligned}
$$

This proves that for all $s, t \in[0, T], \omega \in \Omega \backslash \tilde{\Omega}$ with $s<t$ it holds that

$$
\begin{aligned}
& \left\|\tilde{O}_{t}(\omega)-\tilde{O}_{s}(\omega)\right\|_{V} \\
& \leq \frac{K^{2}(t-s)^{(1-\rho)}}{(1-\rho)}+2^{(\rho+\kappa)} K^{2}(t-s)^{\kappa}\left[\sup _{u \in(0, s)}\left\|(-u A)^{\kappa} e^{u A}\right\|_{L(H)}\right] \\
& \quad \cdot\left[\sup _{u \in(0, T]}\left\|(-u A)^{-\kappa}\left(e^{u A}-\operatorname{Id}_{H}\right)\right\|_{L(H)}\right] \int_{0}^{s}(s-u)^{-(\rho+\kappa)} d u \\
& \leq \frac{K^{2}(t-s)^{(1-\rho)}}{(1-\rho)}+\frac{2^{(\rho+\kappa)} K^{4}(t-s)^{\kappa} s^{(1-\rho-\kappa)}}{(1-\rho-\kappa)} \leq(t-s)^{\kappa} T^{(1-\rho-\kappa)}\left[\frac{K^{2}}{(1-\rho)}+\frac{2^{(\rho+\kappa)} K^{4}}{(1-\rho-\kappa)}\right] .
\end{aligned}
$$

Hence, we obtain that for all $\omega \in \Omega \backslash \tilde{\Omega}, r \in[0, \kappa]$ it holds that

$$
\begin{aligned}
\sup _{0 \leq s<t \leq T} \frac{s\left\|\tilde{O}_{t}(\omega)-\tilde{O}_{s}(\omega)\right\|_{V}}{(t-s)^{r}} & \leq \sup _{0 \leq s<t \leq T}\left(s(t-s)^{(\kappa-r)} T^{(1-\rho-\kappa)}\left[\frac{K^{2}}{(1-\rho)}+\frac{2^{(\rho+\kappa)} K^{4}}{(1-\rho-\kappa)}\right]\right) \\
& \leq T^{(2-\rho-r)}\left[\frac{K^{2}}{(1-\rho)}+\frac{2^{(\rho+\kappa)} K^{4}}{(1-\rho-\kappa)}\right]<\infty
\end{aligned}
$$

Combining this with the fact that $\forall \omega \in \tilde{\Omega}: \lim _{\sup _{r}{ }_{0}} \sup _{0 \leq s<t \leq T} \frac{s\left\|\tilde{O}_{t}(\omega)-\tilde{O}_{s}(\omega)\right\|_{V}}{(t-s)^{r}}<\infty$ ensures that for all $\omega \in \Omega$ it holds that

$$
\limsup _{r \searrow 0} \sup _{0 \leq s<t \leq T} \frac{s\left\|\tilde{O}_{t}(\omega)-\tilde{O}_{s}(\omega)\right\|_{V}}{(t-s)^{r}}<\infty .
$$

In the next step note that for all $t \in[0, T]$ it holds that $\mathbb{P}\left(\tilde{O}_{t}=O_{t}\right) \geq \mathbb{P}(\tilde{\Omega})=1$. This and (91) show that

$$
\begin{aligned}
& \sup _{M \in \mathbb{N}} \sup _{t \in[0, T]}\left[\left|M\lfloor t\rfloor_{T / M}\right|^{\theta}\left\|\tilde{O}_{t}-\tilde{O}_{\lfloor t\rfloor_{T / M}}\right\|_{\mathcal{L}^{2 p}(\mathbb{P} ; V)}+\|\| \tilde{O}_{t}\left\|_{V}+M^{\theta}\right\| \tilde{O}_{t}-\mathcal{O}_{t}^{M}\left\|_{V}\right\|_{\left.\mathcal{L}^{p \max \{2, \varphi, 4 \vartheta, 2 \vartheta \varphi\}(\mathbb{P} ; \mathbb{R})}\right]}\right] \\
& <\infty
\end{aligned}
$$


The triangle inequality hence implies that

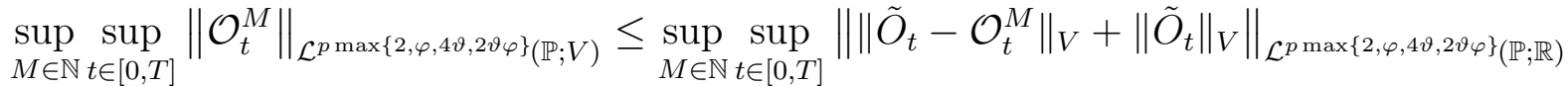

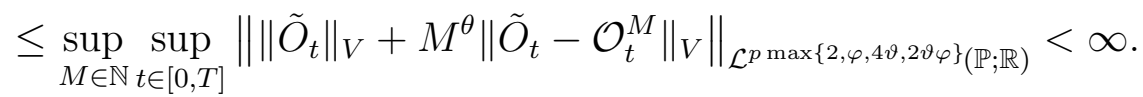

In addition, observe that the assumption that $p \geq \max \left\{2, \frac{1}{\varphi}, \frac{1}{2 \phi} \min \left\{1, \frac{2}{\varphi}, \frac{1}{2 \vartheta}, \frac{1}{\vartheta \varphi}\right\}\right\}$ ensures that $p \max \{2, \varphi, 4 \vartheta, 2 \vartheta \varphi\} \geq 1 / \phi$. Combining (91), (98), and the fact that $\forall t \in[0, T], M \in \mathbb{N}: \mathbb{P}\left(\tilde{\mathcal{X}}_{t}^{M}=\right.$ $\left.\mathcal{X}_{t}^{M}\right)=1$ with Corollary 5.1 hence proves that

$$
\sup _{M \in \mathbb{N}} \sup _{t \in[0, T]}\left\|\tilde{\mathcal{X}}_{t}^{M}\right\|_{\mathcal{L}^{p \max \{2, \varphi, 4 \vartheta, 2 \vartheta \varphi\}(\mathbb{P} ; V)}}=\sup _{M \in \mathbb{N}} \sup _{t \in[0, T]}\left\|\mathcal{X}_{t}^{M}\right\|_{\mathcal{L}^{p \max \{2, \varphi, 4 \vartheta, 2 \vartheta \varphi\}(\mathbb{P} ; V)}}<\infty .
$$

In the next step we combine (91), the fact that $2 p \max \{2, \varphi, 2 \vartheta, \vartheta \varphi\} \geq p \max \{2, \varphi, 4 \vartheta, 2 \vartheta \varphi\} \geq 1 / \phi$, and the fact that $\forall t \in[0, T], M \in \mathbb{N}: \mathbb{P}\left(\tilde{\mathcal{X}}_{t}^{M}=\mathcal{X}_{t}^{M}\right)=1$ with Corollary $[5.2$ to obtain that

$$
\sup _{M \in \mathbb{N}} \sup _{t \in[0, T)}\left\|F\left(\tilde{\mathcal{X}}_{t}^{M}\right)\right\|_{\mathcal{L}^{2 p \max \{2, \varphi, 2 \vartheta, \vartheta \varphi\}(\mathbb{P} ; H)}}=\sup _{M \in \mathbb{N}} \sup _{t \in[0, T)}\left\|F\left(\mathcal{X}_{t}^{M}\right)\right\|_{\mathcal{L}^{2 p \max \{2, \varphi, 2 \vartheta, \vartheta \varphi\}(\mathbb{P} ; H)}}<\infty .
$$

This and (99) imply that

$$
\sup _{M \in \mathbb{N}} \sup _{t \in[0, T]}\left\|\tilde{\mathcal{X}}_{t}^{M}\right\|_{\mathcal{L}^{p \max \{1, \varphi\}(\mathbb{P} ; V)}}+\sup _{M \in \mathbb{N}} \sup _{t \in[0, T)}\|\| \tilde{\mathcal{X}}_{t}^{M}\left\|_{V}^{2 \vartheta}+\right\| F\left(\tilde{\mathcal{X}}_{t}^{M}\right)\left\|_{H}^{2}\right\|_{\mathcal{L}^{p \max \{2, \varphi\}(\mathbb{P} ; \mathbb{R})}}<\infty .
$$

Combining this, (91), (96)), and (97) with Proposition 4.4 (with $C=c, L=c, \alpha=\vartheta \chi, \mathcal{V}(v)=$ $\|v\|_{V}^{1 / \chi}, X_{t}=\tilde{X}_{t}, O_{t}=\tilde{O}_{t}, \bar{X}_{t}=\tilde{X}_{t}-\tilde{O}_{t}, \mathcal{O}_{t}^{M}=\mathcal{O}_{t}^{M}, \mathcal{X}_{t}^{M}=\tilde{\mathcal{X}}_{t}^{M}, \overline{\mathcal{X}}_{t}^{M}=\tilde{\mathcal{X}}_{t}^{M}-\mathcal{O}_{t}^{M}$, and $\mathbf{X}_{t}^{M}=\int_{0}^{t} e^{(t-s) A} F\left(\tilde{\mathcal{X}}_{[s\rfloor_{T / M}^{M}}^{M}\right) d s$ for $v \in V, t \in[0, T], M \in \mathbb{N}$ in the notation of Proposition 4.4) ensures that $\sup _{M \in \mathbb{N}} \sup _{t \in[0, T]}\left[\left\|\tilde{X}_{t}\right\|_{\mathcal{L}^{p}(\mathbb{P} ; H)}+M^{\min \{\vartheta \chi, \varrho, \theta\}}\left\|\tilde{X}_{t}-\tilde{\mathcal{X}}_{t}^{M}\right\|_{\mathcal{L}^{p}(\mathbb{P} ; H)}\right]<\infty$. This, the fact that $\forall t \in[0, T]: \mathbb{P}\left(X_{t}=\tilde{X}_{t}\right) \geq \mathbb{P}(\tilde{\Omega})=1$, the fact that $\forall t \in[0, T], M \in \mathbb{N}: \mathbb{P}\left(\mathcal{X}_{t}^{M}=\tilde{\mathcal{X}}_{t}^{M}\right)=1$, and (99) establish (92). The proof of Theorem 5.3 is thus completed.

\section{Examples}

\subsection{Setting}

Let $T, \nu \in(0, \infty), d \in \mathbb{N}, n \in\{1,3,5, \ldots\}, a_{0}, a_{1}, \ldots, a_{n-1}, b_{1} \in \mathbb{R}, a_{n} \in(-\infty, 0), b_{2} \in\left(b_{1}, \infty\right), \mathcal{D}=$ $\left(b_{1}, b_{2}\right)^{d} \subseteq \mathbb{R}^{d},\left(H,\langle\cdot, \cdot\rangle_{H},\|\cdot\|_{H}\right)=\left(L^{2}\left(\lambda_{\mathcal{D}} ; \mathbb{R}\right),\langle\cdot, \cdot\rangle_{L^{2}\left(\lambda_{\mathcal{D}} ; \mathbb{R}\right)},\|\cdot\|_{L^{2}\left(\lambda_{\mathcal{D}} ; \mathbb{R}\right)}\right),\left(V,\|\cdot\|_{V}\right)=\left(L^{2 n^{2}}\left(\lambda_{\mathcal{D}} ; \mathbb{R}\right)\right.$, $\left.\|\cdot\|_{L^{2 n^{2}}\left(\lambda_{\mathcal{D}} ; \mathbb{R}\right)}\right), F \in \mathcal{C}(V, H), \mathbf{F} \in \mathcal{C}\left(\mathcal{L}^{2 n^{2}}\left(\lambda_{D} ; \mathbb{R}\right), \mathcal{L}^{2}\left(\lambda_{D} ; \mathbb{R}\right)\right)$ satisfy for all $v \in V, w \in \mathcal{L}^{2 n^{2}}\left(\lambda_{D} ; \mathbb{R}\right)$ that $F(v)=\sum_{k=0}^{n} a_{k} v^{k}$ and $\mathbf{F}(w)=\sum_{k=0}^{n} a_{k} w^{k}$, let $A: D(A) \subseteq H \rightarrow H$ be the Laplacian with Dirichlet boundary conditions on $H$ times the real number $\nu$, let $\left(H_{r},\langle\cdot, \cdot\rangle_{H_{r}},\|\cdot\|_{H_{r}}\right), r \in \mathbb{R}$, be a family of interpolation spaces associated to $-A$ (see, e.g., Definition 3.5.26 in [23]), let $(\Omega, \mathcal{F}, \mathbb{P}$ ) be a probability space with a normal filtration $\left(\mathcal{F}_{t}\right)_{t \in[0, T]}$, and let $(\dot{\varepsilon}):\left\{[v]_{\lambda_{D}, \mathcal{B}(\mathbb{R})} \in H:(v: \mathcal{D} \rightarrow\right.$ $\mathbb{R}$ is a uniformly continuous function $)\} \rightarrow \mathcal{C}(\mathcal{D}, \mathbb{R})$ be the function with the property that for all uniformly continuous functions $v: \mathcal{D} \rightarrow \mathbb{R}$ it holds that $\underline{[v]_{\lambda_{D}, \mathcal{B}(\mathbb{R})}}=v$. 


\subsection{Auxiliary lemmas}

In this subsection we establish a few elementary and partially well-known auxiliary lemmas that we need to apply Theorem 5.3 above (see Corollary 6.14 and Corollary 6.16 below for details).

Lemma 6.1. Assume the setting in Section 6.1 and let $q \in\{2,4,6, \ldots\}, v \in L^{q}\left(\lambda_{\mathcal{D}} ; \mathbb{R}\right), t \in[0, T]$. Then $\max \left\{\left\|e^{t A} v\right\|_{L^{q}\left(\lambda_{\mathcal{D}} ; \mathbb{R}\right)},\left\|\left(\operatorname{Id}_{H}-t A\right)^{-1} v\right\|_{L^{q}\left(\lambda_{\mathcal{D}} ; \mathbb{R}\right)}\right\} \leq\|v\|_{L^{q}\left(\lambda_{\mathcal{D}} ; \mathbb{R}\right)}$.

Proof of Lemma 6.1. Throughout this proof assume w.l.o.g. that $t \in(0, T]$ (otherwise the proof is clear). Note that the fundamental theorem of calculus implies for all $\varepsilon \in(0, t)$ that

$$
\left\|e^{t A} v\right\|_{L^{q}\left(\lambda_{\mathcal{D}} ; \mathbb{R}\right)}^{q}=\left\|e^{\varepsilon A} v\right\|_{L^{q}\left(\lambda_{\mathcal{D}} ; \mathbb{R}\right)}^{q}+\int_{\varepsilon}^{t}\left(\frac{\partial}{\partial s}\left\|e^{s A} v\right\|_{L^{q}\left(\lambda_{\mathcal{D}} ; \mathbb{R}\right)}^{q}\right) d s .
$$

Furthermore, integration by parts shows that for all $s \in(0, t)$ it holds that

$$
\begin{aligned}
& \frac{\partial}{\partial s}\left\|e^{s A} v\right\|_{L^{q}\left(\lambda_{\mathcal{D}} ; \mathbb{R}\right)}^{q}=\int_{\mathcal{D}} \frac{\partial}{\partial s}\left[\left(\underline{e^{s A} v}\right)(x)\right]^{q} \lambda_{\mathbb{R}^{d}}(d x)=q \int_{\mathcal{D}}\left[\left(\underline{e^{s A} v}\right)(x)\right]^{(q-1)}\left[\frac{\partial}{\partial s}\left(\underline{e^{s A} v}\right)(x)\right] \lambda_{\mathbb{R}^{d}}(d x) \\
& =\nu q \sum_{k=1}^{d} \int_{\mathcal{D}}\left[\left(\underline{e^{s A} v}\right)\left(x_{1}, \ldots, x_{d}\right)\right]^{(q-1)}\left[\frac{\partial^{2}}{\partial x_{k}^{2}}\left(\underline{e^{s A} v}\right)\left(x_{1}, \ldots, x_{d}\right)\right] \lambda_{\mathbb{R}^{d}}\left(d x_{1}, \ldots, d x_{d}\right) \\
& =-\nu q(q-1) \sum_{k=1}^{d} \int_{\mathcal{D}}\left[\left(\underline{e^{s A} v}\right)\left(x_{1}, \ldots, x_{d}\right)\right]^{(q-2)}\left[\frac{\partial}{\partial x_{k}}\left(\underline{e^{s A} v}\right)\left(x_{1}, \ldots, x_{d}\right)\right]^{2} \lambda_{\mathbb{R}^{d}}\left(d x_{1}, \ldots, d x_{d}\right) \leq 0 .
\end{aligned}
$$

Combining (102) and (103) proves for all $\varepsilon \in(0, t)$ that $\left\|e^{t A} v\right\|_{L^{q}\left(\lambda_{\mathcal{D}} ; \mathbb{R}\right)} \leq\left\|e^{\varepsilon A} v\right\|_{L^{q}\left(\lambda_{\mathcal{D}} ; \mathbb{R}\right)}$. Therefore, we obtain that $\left\|e^{t A} v\right\|_{L^{q}\left(\lambda_{\mathcal{D}} ; \mathbb{R}\right)} \leq\|v\|_{L^{q}\left(\lambda_{\mathcal{D}} ; \mathbb{R}\right)}$. This and the Hille-Yosida theorem (see, e.g., Theorem 1.3.1 in Pazy [37]) imply that $\left\|\left(\operatorname{Id}_{H}-t A\right)^{-1} v\right\|_{L^{q}\left(\lambda_{\mathcal{D}} ; \mathbb{R}\right)} \leq\|v\|_{L^{q}\left(\lambda_{\mathcal{D}} ; \mathbb{R}\right)}$. The proof of Lemma 6.1 is thus completed.

Lemma 6.2. Assume the setting in Section 6.1, let $\eta: \mathbb{N}_{0} \times \mathbb{N}_{0} \rightarrow(0, \infty)$ be a function, and let $q \in\{2 n, 2 n+2,2 n+4, \ldots\}, h \in(0, T], t \in[0, h], u, v \in \mathcal{L}^{n q}\left(\lambda_{\mathcal{D}} ; \mathbb{R}\right), \chi \in\left[0, \frac{1}{2 n}\right]$. Then

$$
\begin{aligned}
& \left\|u+t \mathbb{1}_{\left[0, h^{-\chi}\right]}\left(\|u+v\|_{\mathcal{L}^{n q}\left(\lambda_{\mathcal{D}} ; \mathbb{R}\right)}\right) \mathbf{F}(u+v)\right\|_{\mathcal{L}^{q}\left(\lambda_{\mathcal{D}} ; \mathbb{R}\right)}^{q} \\
& \leq q t\left(a_{n}+\sum_{k=0}^{n} \sum_{j=0}^{\min \{k, n-1\}}\left(\begin{array}{c}
k \\
j
\end{array}\right)\left|a_{k}\right|\left|\eta_{(k, j)}\right|^{\frac{(q+n-1)}{(q+j-1)}}\right)\|u\|_{\mathcal{L}^{(q+n-1)}\left(\lambda_{\mathcal{D}} ; \mathbb{R}\right)}^{(q+n-1)} \\
& \quad+e^{t}\|u\|_{\mathcal{L}^{q}\left(\lambda_{\mathcal{D}} ; \mathbb{R}\right)}^{q}+t \max \left\{1, \lambda_{\mathbb{R}^{d}}(\mathcal{D})\right\} \max \left\{1,\|v\|_{\mathcal{L}^{(q+n-1)}\left(\lambda_{\mathcal{D}} ; \mathbb{R}\right)}^{(q+n-1)}\right\} \\
& \quad \cdot\left(2 q \sum_{k=0}^{n} \sum_{j=0}^{\min \{k, n-1\}}\left(\begin{array}{c}
k \\
j
\end{array}\right)\left|a_{k}\right|\left|\eta_{(k, j)}\right|^{-\frac{(q+n-1)}{(n-j)}}+\left[q(n+1) \max ^{n}\{1, T\} \max _{j \in\{0,1, \ldots, n\}}\left|a_{j}\right|\right]^{q}\right) .
\end{aligned}
$$


Proof of Lemma 6.2. First of all, note that

$$
\begin{aligned}
\left\|u+t \mathbb{1}_{\left[0, h^{-} \chi\right]}\left(\|u+v\|_{\mathcal{L}^{n q}\left(\lambda_{\mathcal{D}} ; \mathbb{R}\right)}\right) \mathbf{F}(u+v)\right\|_{\mathcal{L}^{q}\left(\lambda_{\mathcal{D}} ; \mathbb{R}\right)}^{q} \\
=\int_{\mathcal{D}}\left|u(x)+t \mathbb{1}_{\left[0, h^{-\chi}\right]}\left(\|u+v\|_{\mathcal{L}^{n q}\left(\lambda_{\mathcal{D}} ; \mathbb{R}\right)}\right)\left(\sum_{k=0}^{n} a_{k}[u(x)+v(x)]^{k}\right)\right|^{q} \lambda_{\mathbb{R}^{d}}(d x) \\
=\|u\|_{\mathcal{L}^{q}\left(\lambda_{\mathcal{D}} ; \mathbb{R}\right)}^{q}+q t \mathbb{1}_{\left[0, h^{-\chi}\right]}\left(\|u+v\|_{\mathcal{L}^{n q}\left(\lambda_{\mathcal{D}} ; \mathbb{R}\right)}\right) \int_{\mathcal{D}}[u(x)]^{(q-1)}\left(\sum_{k=0}^{n} a_{k}[u(x)+v(x)]^{k}\right) \lambda_{\mathbb{R}^{d}}(d x) \\
\quad+\sum_{l=2}^{q}\left(\begin{array}{l}
q \\
l
\end{array}\right) t^{l} \mathbb{1}_{\left[0, h^{-\chi}\right]}\left(\|u+v\|_{\mathcal{L}^{n q}\left(\lambda_{\mathcal{D}} ; \mathbb{R}\right)}\right) \int_{\mathcal{D}}[u(x)]^{(q-l)}\left[\sum_{k=0}^{n} a_{k}[u(x)+v(x)]^{k}\right]^{l} \lambda_{\mathbb{R}^{d}}(d x) .
\end{aligned}
$$

Furthermore, observe that Young's inequality assures that

$$
\begin{aligned}
& q t \mathbb{1}_{\left[0, h^{-\chi}\right]}\left(\|u+v\|_{\mathcal{L}^{n q}\left(\lambda_{\mathcal{D}} ; \mathbb{R}\right)}\right) \int_{\mathcal{D}}[u(x)]^{(q-1)}\left(\sum_{k=0}^{n} a_{k}[u(x)+v(x)]^{k}\right) \lambda_{\mathbb{R}^{d}}(d x) \\
& =q t \mathbb{1}_{\left[0, h^{-\chi}\right]}\left(\|u+v\|_{\mathcal{L}^{n q}\left(\lambda_{\mathcal{D}} ; \mathbb{R}\right)}\right) \sum_{k=0}^{n} \sum_{j=0}^{k}\left(\begin{array}{c}
k \\
j
\end{array}\right) a_{k} \int_{\mathcal{D}}[u(x)]^{(q+j-1)}[v(x)]^{(k-j)} \lambda_{\mathbb{R}^{d}}(d x) \\
& \leq q t a_{n}\|u\|_{\mathcal{L}^{(q+n-1)}\left(\lambda_{\mathcal{D}} ; \mathbb{R}\right)}^{(q+n-1)}+q t \sum_{k=0}^{n} \sum_{j=0}^{\min \{k, n-1\}}\left(\begin{array}{c}
k \\
j
\end{array}\right)\left|a_{k}\right| \int_{\mathcal{D}} \eta_{(k, j)}|u(x)|^{(q+j-1)} \frac{|v(x)|^{(k-j)}}{\eta_{(k, j)}} \lambda_{\mathbb{R}^{d}}(d x) \\
& \leq q t a_{n}\|u\|_{\mathcal{L}^{(q+n-1)}\left(\lambda_{\mathcal{D}} ; \mathbb{R}\right)}^{(q+n-1)} \\
& +q t \sum_{k=0}^{n} \sum_{j=0}^{\min \{k, n-1\}}\left(\begin{array}{c}
k \\
j
\end{array}\right)\left|a_{k}\right| \int_{\mathcal{D}}\left[\left|\eta_{(k, j)}\right|^{\frac{(q+n-1)}{(q+j-1)}}|u(x)|^{(q+n-1)}+\left|\eta_{(k, j)}\right|^{-\frac{(q+n-1)}{(n-j)}}|v(x)|^{\frac{(k-j)(q+n-1)}{(n-j)}}\right] \lambda_{\mathbb{R}^{d}}(d x) .
\end{aligned}
$$

This implies that

$$
\begin{aligned}
& q t \mathbb{1}_{\left[0, h^{-\chi}\right]}\left(\|u+v\|_{\mathcal{L}^{n q}\left(\lambda_{\mathcal{D}} ; \mathbb{R}\right)}\right) \int_{\mathcal{D}}[u(x)]^{(q-1)}\left(\sum_{k=0}^{n} a_{k}[u(x)+v(x)]^{k}\right) \lambda_{\mathbb{R}^{d}}(d x) \\
& \leq q t\left[a_{n}+\sum_{k=0}^{n} \sum_{j=0}^{\min \{k, n-1\}}\left(\begin{array}{l}
k \\
j
\end{array}\right)\left|a_{k}\right|\left|\eta_{(k, j)}\right|^{\frac{(q+n-1)}{(q+j-1)}}\right]\|u\|_{\mathcal{L}^{(q+n-1)}\left(\lambda_{\mathcal{D}} ; \mathbb{R}\right)}^{(q+n-1)} \\
& +q t \sum_{k=0}^{n} \sum_{j=0}^{\min \{k, n-1\}}\left(\begin{array}{l}
k \\
j
\end{array}\right)\left|a_{k}\right|\left|\eta_{(k, j)}\right|^{-\frac{(q+n-1)}{(n-j)}} \int_{\mathcal{D}} \max \left\{1,|v(x)|^{(q+n-1)}\right\} \lambda_{\mathbb{R}^{d}}(d x) \\
& \leq q t\left[a_{n}+\sum_{k=0}^{n} \sum_{j=0}^{\min \{k, n-1\}}\left(\begin{array}{l}
k \\
j
\end{array}\right)\left|a_{k}\right|\left|\eta_{(k, j)}\right|^{\frac{(q+n-1)}{(q+j-1)}}\right]\|u\|_{\mathcal{L}^{(q+n-1)}\left(\lambda_{\mathcal{D}} ; \mathbb{R}\right)}^{(q+n-1)} \\
& +q t \sum_{k=0}^{n} \sum_{j=0}^{\min \{k, n-1\}}\left(\begin{array}{l}
k \\
j
\end{array}\right)\left|a_{k}\right|\left|\eta_{(k, j)}\right|^{-\frac{(q+n-1)}{(n-j)}}\left(\lambda_{\mathbb{R}^{d}}(\mathcal{D})+\|v\|_{\mathcal{L}^{(q+n-1)}\left(\lambda_{\mathcal{D}} ; \mathbb{R}\right)}^{(q+n-1)}\right) \text {. }
\end{aligned}
$$


Moreover, note that the fact that $\forall m \in \mathbb{N},\left(z_{l}\right)_{l \in\{0,1, \ldots, m\}} \subseteq \mathbb{R}, r \in[0, \infty):\left|z_{0}+z_{1}+\ldots+z_{m}\right|^{r} \leq$ $(m+1)^{[r-1]^{+}}\left(\left|z_{0}\right|^{r}+\left|z_{1}\right|^{r}+\ldots+\left|z_{m}\right|^{r}\right)$ and Hölder's inequality imply that

$$
\begin{aligned}
& \sum_{l=2}^{q}\left(\begin{array}{l}
q \\
l
\end{array}\right) t^{l} \mathbb{1}_{\left[0, h^{-}-\right]}\left(\|u+v\|_{\mathcal{L}^{n q}\left(\lambda_{\mathcal{D}} ; \mathbb{R}\right)}\right) \int_{\mathcal{D}}[u(x)]^{(q-l)}\left[\sum_{k=0}^{n} a_{k}[u(x)+v(x)]^{k}\right]^{l} \lambda_{\mathbb{R}^{d}}(d x) \\
& \leq \sum_{l=2}^{q} \sum_{k=0}^{n}\left(\begin{array}{l}
q \\
l
\end{array}\right)(n+1)^{(l-1)} t^{l}\left|a_{k}\right|^{l} \mathbb{1}_{\left[0, h^{-\chi}\right]}\left(\|u+v\|_{\mathcal{L}^{n q}\left(\lambda_{\mathcal{D}} ; \mathbb{R}\right)}\right) \int_{\mathcal{D}}|u(x)|^{(q-l)}|u(x)+v(x)|^{k l} \lambda_{\mathbb{R}^{d}}(d x) \\
& \leq \sum_{l=2}^{q}\left(\begin{array}{l}
q \\
l
\end{array}\right)(n+1)^{(l-1)} t^{l}\left|a_{0}\right|^{l} \mathbb{1}_{\left[0, h^{-\chi}\right]}\left(\|u+v\|_{\mathcal{L}^{n q}\left(\lambda_{\mathcal{D}} ; \mathbb{R}\right)}\right)\|u\|_{\mathcal{L}^{q}\left(\lambda_{\mathcal{D}} ; \mathbb{R}\right)}^{(q-l)}\left|\lambda_{\mathbb{R}^{d}}(\mathcal{D})\right|^{\frac{l}{q}} \\
& \quad+\sum_{l=2}^{q} \sum_{k=1}^{n}\left(\begin{array}{l}
q \\
l
\end{array}\right)(n+1)^{(l-1)} t^{l}\left|a_{k}\right|^{l} \mathbb{1}_{\left[0, h^{-\chi}\right]}\left(\|u+v\|_{\mathcal{L}^{n q}\left(\lambda_{\mathcal{D}} ; \mathbb{R}\right)}\right)\|u\|_{\mathcal{L}^{q}\left(\lambda_{\mathcal{D}} ; \mathbb{R}\right)}^{(q-l)}\|u+v\|_{\mathcal{L}^{k q}\left(\lambda_{\mathcal{D}} ; \mathbb{R}\right)}^{k l} .
\end{aligned}
$$

Again Hölder's inequality hence shows that

$$
\begin{aligned}
& \sum_{l=2}^{q}\left(\begin{array}{l}
q \\
l
\end{array}\right) t^{l} \mathbb{1}_{\left[0, h^{-\chi}\right]}\left(\|u+v\|_{\mathcal{L}^{n q}\left(\lambda_{\mathcal{D}} ; \mathbb{R}\right)}\right) \int_{\mathcal{D}}[u(x)]^{(q-l)}\left[\sum_{k=0}^{n} a_{k}[u(x)+v(x)]^{k}\right]^{l} \lambda_{\mathbb{R}^{d}}(d x) \\
& \leq \sum_{l=2}^{q}\left(\begin{array}{l}
q \\
l
\end{array}\right)(n+1)^{(l-1)} t^{l}\left|a_{0}\right|^{l}\|u\|_{\mathcal{L}^{q}\left(\lambda_{\mathcal{D}} ; \mathbb{R}\right)}^{(q-l)}\left|\lambda_{\mathbb{R}^{d}}(\mathcal{D})\right|^{\frac{l}{q}} \\
& +\sum_{l=2}^{q} \sum_{k=1}^{n}\left(\begin{array}{l}
q \\
l
\end{array}\right)(n+1)^{(l-1)} t^{l}\left|a_{k}\right|^{l} \mathbb{1}_{\left[0, h^{-\chi}\right]}\left(\|u+v\|_{\mathcal{L}^{n q}\left(\lambda_{\mathcal{D}} ; \mathbb{R}\right)}\right)\|u\|_{\mathcal{L}^{q}\left(\lambda_{\mathcal{D}} ; \mathbb{R}\right)}^{(q-l)}\|u+v\|_{\mathcal{L}^{n q}\left(\lambda_{\mathcal{D}} ; \mathbb{R}\right)}^{k l}\left|\lambda_{\mathbb{R}^{d}}(\mathcal{D})\right|^{\frac{l(n-k)}{n q}} \\
& \leq \sum_{l=2}^{q}\left(\begin{array}{l}
q \\
l
\end{array}\right)(n+1)^{(l-1)} t^{l}\left[\max _{j \in\{0, \ldots, n\}}\left|a_{j}\right|^{l}\right]\left[\|u\|_{\mathcal{L}^{q}\left(\lambda_{\mathcal{D}} ; \mathbb{R}\right)}^{(q-l)}\left|\lambda_{\mathbb{R}^{d}}(\mathcal{D})\right|^{\frac{l}{q}}+\sum_{k=1}^{n} h^{-k l \chi}\|u\|_{\mathcal{L}^{q}\left(\lambda_{\mathcal{D}} ; \mathbb{R}\right)}^{(q-l)}\left|\lambda_{\mathbb{R}^{d}}(\mathcal{D})\right|^{\frac{l(n-k)}{n q}}\right] \\
& \leq t \sum_{l=2}^{q}\left(\begin{array}{l}
q \\
l
\end{array}\right)(n+1)^{(l-1)}\left[\max _{j \in\{0, \ldots, n\}}\left|a_{j}\right|^{l}\right] \sum_{k=0}^{n} h^{(l-1-k l \chi)}\|u\|_{\mathcal{L}^{q}\left(\lambda_{\mathcal{D}} ; \mathbb{R}\right)}^{(q-l)}\left|\lambda_{\mathbb{R}^{d}}(\mathcal{D})\right|^{\frac{l(n-k)}{n q}} .
\end{aligned}
$$

Young's inequality therefore ensures that

$$
\begin{aligned}
& \sum_{l=2}^{q}\left(\begin{array}{l}
q \\
l
\end{array}\right) t^{l} \mathbb{1}_{\left[0, h^{-\chi}\right]}\left(\|u+v\|_{\mathcal{L}^{n q}\left(\lambda_{\mathcal{D}} ; \mathbb{R}\right)}\right) \int_{\mathcal{D}}[u(x)]^{(q-l)}\left[\sum_{k=0}^{n} a_{k}[u(x)+v(x)]^{k}\right]^{l} \lambda_{\mathbb{R}^{d}}(d x) \\
& \leq t \sum_{l=2}^{q}\left(\begin{array}{l}
q \\
l
\end{array}\right) \frac{q^{l}(n+1)^{l}}{q^{l}(n+1)}\left[\max _{j \in\{0, \ldots, n\}}\left|a_{j}\right|^{l}\right] \sum_{k=0}^{n} \max \left\{1, T^{(l-1)}\right\}\|u\|_{\mathcal{L}^{q}\left(\lambda_{\mathcal{D}} ; \mathbb{R}\right)}^{(q-l)}\left|\lambda_{\mathbb{R}^{d}}(\mathcal{D})\right|^{\frac{l(n-k)}{n q}} \\
& \leq t \sum_{l=2}^{q}\left(\begin{array}{l}
q \\
l
\end{array}\right) \frac{1}{q^{l}(n+1)} \sum_{k=0}^{n}\left(\|u\|_{\mathcal{L}^{q}\left(\lambda_{\mathcal{D}} ; \mathbb{R}\right)}^{q}+q^{q}(n+1)^{q} \max \left\{1, T^{\frac{q(l-1)}{l}}\right\}\left[\max _{j \in\{0,1, \ldots, n\}}\left|a_{j}\right|^{q}\right]\left|\lambda_{\mathbb{R}^{d}}(\mathcal{D})\right|^{\frac{(n-k)}{n}}\right) \\
& \leq t \sum_{l=2}^{q} \frac{1}{l !}\left(\|u\|_{\mathcal{L}^{q}\left(\lambda_{\mathcal{D}} ; \mathbb{R}\right)}^{q}+\left[q(n+1) \max \{1, T\} \max _{j \in\{0,1, \ldots, n\}}\left|a_{j}\right|\right]^{q} \max \left\{1, \lambda_{\mathbb{R}^{d}}(\mathcal{D})\right\}\right) .
\end{aligned}
$$


Combining (105), (107), and (110) yields that

$$
\begin{aligned}
& \left\|u+t \mathbb{1}_{\left[0, h^{-\chi}\right]}\left(\|u+v\|_{\mathcal{L}^{n q}\left(\lambda_{\mathcal{D}} ; \mathbb{R}\right)}\right) \mathbf{F}(u+v)\right\|_{\mathcal{L}^{q}\left(\lambda_{\mathcal{D}} ; \mathbb{R}\right)}^{q} \\
& \leq\|u\|_{\mathcal{L}^{q}\left(\lambda_{\mathcal{D}} ; \mathbb{R}\right)}^{q}+q t\left[a_{n}+\sum_{k=0}^{n} \sum_{j=0}^{\min \{k, n-1\}}\left(\begin{array}{c}
k \\
j
\end{array}\right)\left|a_{k}\right|\left|\eta_{(k, j)}\right|^{\frac{(q+n-1)}{(q+j-1)}}\right]\|u\|_{\mathcal{L}^{(q+n-1)}\left(\lambda_{\mathcal{D}} ; \mathbb{R}\right)}^{(q+n-1)} \\
& \quad+q t \sum_{k=0}^{n} \sum_{j=0}^{\min \{k, n-1\}}\left(\begin{array}{c}
k \\
j
\end{array}\right)\left|a_{k}\right|\left|\eta_{(k, j)}\right|^{-\frac{(q+n-1)}{(n-j)}}\left(\lambda_{\mathbb{R}^{d}}(\mathcal{D})+\|v\|_{\mathcal{L}^{(q+n-1)}\left(\lambda_{\mathcal{D}} ; \mathbb{R}\right)}^{(q+n-1)}\right) \\
& \quad+t \sum_{l=2}^{q} \frac{1}{l !}\left(\|u\|_{\mathcal{L}^{q}\left(\lambda_{\mathcal{D}} ; \mathbb{R}\right)}^{q}+\left[q(n+1) \max \{1, T\} \max _{j \in\{0,1, \ldots, n\}}\left|a_{j}\right|\right]^{q} \max \left\{1, \lambda_{\mathbb{R}^{d}}(\mathcal{D})\right\}\right) .
\end{aligned}
$$

The fact that $\sum_{l=2}^{q} \frac{1}{l !}=\left(\sum_{l=0}^{q} \frac{1}{l !}\right)-2 \leq e-2<1$ hence assures that

$$
\begin{aligned}
& \left\|u+t \mathbb{1}_{\left[0, h^{-\chi}\right]}\left(\|u+v\|_{\mathcal{L}^{n q}\left(\lambda_{\mathcal{D}} ; \mathbb{R}\right)}\right) \mathbf{F}(u+v)\right\|_{\mathcal{L}^{q}\left(\lambda_{\mathcal{D}} ; \mathbb{R}\right)}^{q} \\
& \leq(1+t)\|u\|_{\mathcal{L}^{q}\left(\lambda_{\mathcal{D}} ; \mathbb{R}\right)}^{q}+q t\left[a_{n}+\sum_{k=0}^{n} \sum_{j=0}^{\min \{k, n-1\}}\left(\begin{array}{c}
k \\
j
\end{array}\right)\left|a_{k}\right|\left|\eta_{(k, j)}\right|^{\frac{(q+n-1)}{(q+j-1)}}\right]\|u\|_{\mathcal{L}^{(q+n-1)}\left(\lambda_{\mathcal{D}} ; \mathbb{R}\right)}^{(q+n-1)} \\
& \quad+t \max \left\{1, \lambda_{\mathbb{R}^{d}}(\mathcal{D}),\|v\|_{\mathcal{L}^{(q+n-1)}\left(\lambda_{\mathcal{D}} ; \mathbb{R}\right)}^{(q+n-1)}\right\} \\
& \quad\left(2 q \sum_{k=0}^{n} \sum_{j=0}^{\min \{k, n-1\}}\left(\begin{array}{c}
k \\
j
\end{array}\right)\left|a_{k}\right|\left|\eta_{(k, j)}\right|^{-\frac{(q+n-1)}{(n-j)}}+\left[q(n+1) \max \{1, T\} \max _{j \in\{0,1, \ldots, n\}}\left|a_{j}\right|\right]^{q}\right) .
\end{aligned}
$$

This and the fact that $1+t \leq e^{t}$ complete the proof of Lemma 6.2.

Corollary 6.3. Assume the setting in Section 6.1 and let $q \in\{2 n, 2 n+2,2 n+4, \ldots\}$. Then there exists a real number $K \in(0, \infty)$ such that for all $h \in(0, T], t \in[0, h], u, v \in L^{n q}\left(\lambda_{\mathcal{D}} ; \mathbb{R}\right), \chi \in\left[0, \frac{1}{2 n}\right]$ it holds that

$$
\begin{aligned}
& \max \left\{\left\|e^{t A}\left[u+t \mathbb{1}_{\left[0, h^{-\chi}\right]}\left(\|u+v\|_{L^{n q}\left(\lambda_{\mathcal{D}} ; \mathbb{R}\right)}\right) F(u+v)\right]\right\|_{L^{q}\left(\lambda_{\mathcal{D}} ; \mathbb{R}\right)}^{q},\right. \\
& \left.\quad\left\|\left(\operatorname{Id}_{H}-t A\right)^{-1}\left[u+t \mathbb{1}_{\left[0, h^{-\chi}\right]}\left(\|u+v\|_{L^{n q}\left(\lambda_{\mathcal{D}} ; \mathbb{R}\right)}\right) F(u+v)\right]\right\|_{L^{q}\left(\lambda_{\mathcal{D}} ; \mathbb{R}\right)}^{q}\right\} \\
& \leq e^{t}\left[\|u\|_{L^{q}\left(\lambda_{\mathcal{D}} ; \mathbb{R}\right)}^{q}+t K \max \left\{1,\|v\|_{L^{(q+n-1)}\left(\lambda_{\mathcal{D}} ; \mathbb{R}\right)}^{(q+n-1)}\right\}\right] .
\end{aligned}
$$

Proof of Corollary 6.3. Combining Lemma 6.1 and Lemma 6.2 implies (113). The proof of Corollary 6.3 is thus completed.

Lemma 6.4. Assume the setting in Section 6.1 and let $t \in(0, \infty), \kappa \in[0,2], j \in\{2,3, \ldots\}$. Then $\left\|(-j t A)^{\kappa}\left(\operatorname{Id}_{H}-t A\right)^{-j}\right\|_{L(H)} \leq 4$.

Proof of Lemma 6.4. Note that the assumption that $A: D(A) \subseteq H \rightarrow H$ is the Laplacian with Dirichlet boundary conditions on $H$ ensures that there exists an orthonormal basis $\mathbb{B} \subseteq H$ of $H$ and a mapping $\lambda: \mathbb{B} \rightarrow(0, \infty)$ such that $D(A)=\left\{v \in H: \sum_{b \in \mathbb{B}}\left|\lambda_{b}\langle b, v\rangle_{H}\right|^{2}<\infty\right\}$ and such that 
for all $v \in D(A)$ it holds that $A v=\sum_{b \in \mathbb{B}}-\lambda_{b}\langle b, v\rangle_{H} b$. Next observe that for all $x \in[0, \infty)$ it holds that

$$
(1+x)^{j} \geq 1+\frac{j(j-1)}{2} x^{2}=1+\frac{j}{2}\left[\left(\frac{j}{2}+\left(\frac{j}{2}-1\right)\right) x^{2}\right] \geq 1+\frac{j^{2} x^{2}}{4} \geq \frac{(j x)^{\kappa}}{4} .
$$

This implies that

$$
\left\|(-j t A)^{\kappa}\left(\operatorname{Id}_{H}-t A\right)^{-j}\right\|_{L(H)}=\sup _{b \in \mathbb{B}}\left[\frac{\left(j t \lambda_{b}\right)^{\kappa}}{\left(1+t \lambda_{b}\right)^{j}}\right] \leq \sup _{x \in[0, \infty)}\left(\frac{(j x)^{\kappa}}{(1+x)^{j}}\right) \leq 4 .
$$

The proof of Lemma 6.4 is thus completed.

Lemma 6.5. Assume the setting in Section 6.1 and let $q \in[2, \infty), \rho \in[d / 4-d / 2 q, \infty)$ satisfy $d / 4-d / 2 q<1$. Then $\sup _{M \in \mathbb{N}} \sup _{0 \leq s<t \leq T}\left[t^{\rho}\left\|e^{t A}\right\|_{L\left(H, L^{q}\left(\lambda_{\mathcal{D}} ; \mathbb{R}\right)\right)}+(t-s)^{\rho}\left\|e^{\left(t-\lfloor s\rfloor_{T / M}\right) A}\right\|_{L\left(H, L^{q}\left(\lambda_{\mathcal{D}} ; \mathbb{R}\right)\right)}\right]<\infty$ and

$$
\sup _{M \in \mathbb{N}} \sup _{0 \leq s<t \leq T}(t-s)^{\rho}\left\|\left(\operatorname{Id}_{H}-\left(t-\lfloor t\rfloor_{T / M}\right) A\right)^{-1}\left(\operatorname{Id}_{H}-\frac{T}{M} A\right)^{\left(\lfloor s\rfloor_{T / M}-\lfloor t\rfloor_{T / M}\right) \frac{M}{T}}\right\|_{L\left(H, L^{q}\left(\lambda_{\mathcal{D}} ; \mathbb{R}\right)\right)}<\infty .
$$

Proof of Lemma 6.5. Note that the fact that for all $r \in[0, \infty)$ it holds that $H_{r} \subseteq W^{2 r, 2}(\mathcal{D}, \mathbb{R})$ continuously (cf., e.g., (A.46) in Da Prato \& Zabczyk [8] and Lunardi [33]) and the fact that for all $r \in[d / 4-d / 2 q, \infty)$ it holds that $W^{2 r, 2}(\mathcal{D}, \mathbb{R}) \subseteq W^{0, q}(\mathcal{D}, \mathbb{R})=L^{q}\left(\lambda_{\mathcal{D}} ; \mathbb{R}\right)$ continuously imply that there exists a real number $C \in(0, \infty)$ such that for all $v \in H_{(d / 4-d / 2 q)}$ it holds that $\|v\|_{L^{q}\left(\lambda_{\mathcal{D}} ; \mathbb{R}\right)} \leq$ $C\|v\|_{H_{(d / 4-d / 2 q)}}$. This and the fact that $\forall s \in[0, \infty), \kappa \in[0,1]:\left\|(-s A)^{\kappa} e^{s A}\right\|_{L(H)} \leq 1$ prove that for all $t \in(0, T]$ it holds that

$$
\begin{aligned}
& \sup _{v \in H \backslash\{0\}}\left[\frac{t^{\rho}\left\|e^{t A} v\right\|_{L^{q}\left(\lambda_{\mathcal{D}} ; \mathbb{R}\right)}}{\|v\|_{H}}\right] \\
& \leq \sup _{v \in H \backslash\{0\}}\left[\frac{C t^{\rho}\left\|e^{t A} v\right\|_{H_{(d / 4-d / 2 q)}}}{\|v\|_{H}}\right]=\sup _{v \in H \backslash\{0\}}\left[\frac{C t^{\rho}\left\|(-A)^{(d / 4-d / 2 q)} e^{t A} v\right\|_{H}}{\|v\|_{H}}\right] \\
& \leq C t^{(\rho-[d / 4-d / 2 q])}\left\|(-t A)^{(d / 4-d / 2 q)} e^{t A}\right\|_{L(H)} \leq C t^{(\rho-[d / 4-d / 2 q])} .
\end{aligned}
$$

Hence, we obtain that $\sup _{t \in(0, T]} t^{\rho}\left\|e^{t A}\right\|_{L\left(H, L^{q}\left(\lambda_{\mathcal{D}} ; \mathbb{R}\right)\right)} \leq C T^{(\rho-[d / 4-d / 2 q])}<\infty$. This shows that

$$
\begin{aligned}
& \sup _{M \in \mathbb{N}} \sup _{0 \leq s<t \leq T}(t-s)^{\rho}\left\|e^{\left(t-\lfloor s\rfloor_{T / M}\right) A}\right\|_{L\left(H, L^{q}\left(\lambda_{\mathcal{D}} ; \mathbb{R}\right)\right)} \\
& \leq \sup _{M \in \mathbb{N}} \sup _{0 \leq s<t \leq T}\left(t-\lfloor s\rfloor_{T / M}\right)^{\rho}\left\|e^{\left(t-\lfloor s\rfloor_{T / M}\right) A}\right\|_{L\left(H, L^{q}\left(\lambda_{\mathcal{D}} ; \mathbb{R}\right)\right)} \leq \sup _{t \in(0, T]} t^{\rho}\left\|e^{t A}\right\|_{L\left(H, L^{q}\left(\lambda_{\mathcal{D}} ; \mathbb{R}\right)\right)}<\infty .
\end{aligned}
$$

In addition, note that the fact that $\forall x, y \in \mathbb{R}, r \in(0, \infty):|x+y|^{r} \leq 2^{[r-1]^{+}}\left(|x|^{r}+|y|^{r}\right)$ implies 
that for all $s, t \in[0, T], M \in \mathbb{N}$ with $s<t$ it holds that

$$
\begin{aligned}
& \sup _{v \in H \backslash\{0\}}\left[\frac{(t-s)^{\rho}}{\|v\|_{H}}\left\|\left(\operatorname{Id}_{H}-\left(t-\lfloor t\rfloor_{T / M}\right) A\right)^{-1}\left(\operatorname{Id}_{H}-\frac{T}{M} A\right)^{\left(\lfloor s\rfloor_{T / M}-\lfloor t\rfloor_{T / M}\right) \frac{M}{T}} v\right\|_{L^{q}\left(\lambda_{\mathcal{D}} ; \mathbb{R}\right)}\right] \\
& \leq \sup _{v \in H \backslash\{0\}}\left[\frac{C(t-s)^{\rho}}{\|v\|_{H}}\left\|\left(\operatorname{Id}_{H}-\left(t-\lfloor t\rfloor_{T / M}\right) A\right)^{-1}\left(\operatorname{Id}_{H}-\frac{T}{M} A\right)^{\left(\lfloor s\rfloor_{T / M}-\lfloor t\rfloor_{T / M}\right) \frac{M}{T}} v\right\|_{H_{(d / 4-d / 2 q)}}\right] \\
& =\sup _{v \in H \backslash\{0\}}\left[\frac{C(t-s)^{\rho}}{\|v\|_{H}}\left\|(-A)^{(d / 4-d / 2 q)}\left(\operatorname{Id}_{H}-\left(t-\lfloor t\rfloor_{T / M}\right) A\right)^{-1}\left(\operatorname{Id}_{H}-\frac{T}{M} A\right)^{\left(\lfloor s\rfloor_{T / M}-\lfloor t\rfloor_{T / M}\right) \frac{M}{T}} v\right\|_{H}\right] \\
& \leq C\left(t-\lfloor s\rfloor_{T / M}\right)^{\rho}\left\|(-A)^{(d / 4-d / 2 q)}\left(\operatorname{Id}_{H}-\left(t-\lfloor t\rfloor_{T / M}\right) A\right)^{-1}\left(\operatorname{Id}_{H}-\frac{T}{M} A\right)^{\left(\lfloor s\rfloor_{T / M}-\lfloor t\rfloor_{T / M}\right) \frac{M}{T}}\right\|_{L(H)} \\
& \leq 2^{[\rho-1]^{+}} C\left[\left(t-\lfloor t\rfloor_{T / M}\right)^{\rho}+\left(\lfloor t\rfloor_{T / M}-\lfloor s\rfloor_{T / M}\right)^{\rho}\right] \\
& \cdot\left\|(-A)^{(d / 4-d / 2 q)}\left(\operatorname{Id}_{H}-\left(t-\lfloor t\rfloor_{T / M}\right) A\right)^{-1}\left(\operatorname{Id}_{H}-\frac{T}{M} A\right)^{\left(\lfloor s\rfloor_{T / M}-\lfloor t\rfloor_{T / M}\right) \frac{M}{T}}\right\|_{L(H)} .
\end{aligned}
$$

This assures that

$$
\begin{aligned}
& \sup _{M \in \mathbb{N}} \sup _{0 \leq s<t \leq T}(t-s)^{\rho}\left\|\left(\operatorname{Id}_{H}-\left(t-\lfloor t\rfloor_{T / M}\right) A\right)^{-1}\left(\operatorname{Id}_{H}-\frac{T}{M} A\right)^{\left(\lfloor s\rfloor_{T / M}-\lfloor t\rfloor_{T / M}\right) \frac{M}{T}}\right\|_{L\left(H, L^{q}\left(\lambda_{\mathcal{D}} ; \mathbb{R}\right)\right)} \\
& \leq \sup _{M \in \mathbb{N}} \sup _{0 \leq s<t \leq T} 2^{[\rho-1]^{+}} C\left(t-\lfloor t\rfloor_{T / M}\right)^{\rho}\left\|(-A)^{(d / 4-d / 2 q)}\left(\operatorname{Id}_{H}-\left(t-\lfloor t\rfloor_{T / M}\right) A\right)^{-1}\right\|_{L(H)} \\
& \cdot\left\|\left(\operatorname{Id}_{H}-\frac{T}{M} A\right)^{-1}\right\|_{L(H)}^{\left(\lfloor t\rfloor_{T / M}-\lfloor s\rfloor_{T / M}\right) \frac{M}{T}} \\
& +\sup _{M \in \mathbb{N}} \sup _{t \in\left[\frac{T}{M}, T\right]} \sup _{s \in\left[0,\lfloor t\rfloor_{T / M}\right)} 2^{[\rho-1]^{+}} C\left(\lfloor t\rfloor_{T / M}-\lfloor s\rfloor_{T / M}\right)^{\rho}\left\|\left(\operatorname{Id}_{H}-\left(t-\lfloor t\rfloor_{T / M}\right) A\right)^{-1}\right\|_{L(H)} \\
& \cdot\left\|(-A)^{(d / 4-d / 2 q)}\left(\operatorname{Id}_{H}-\frac{T}{M} A\right)^{\left(\lfloor s\rfloor_{T / M}-\lfloor t\rfloor_{T / M}\right) \frac{M}{T}}\right\|_{L(H)} \text {. }
\end{aligned}
$$

The fact that $\forall s \in[0, \infty), \kappa \in[0,1]:\left\|(-s A)^{\kappa}\left(\operatorname{Id}_{H}-s A\right)^{-1}\right\|_{L(H)} \leq 1$ therefore yields that

$$
\begin{aligned}
& \sup _{M \in \mathbb{N}} \sup _{0 \leq s<t \leq T}(t-s)^{\rho}\left\|\left(\operatorname{Id}_{H}-\left(t-\lfloor t\rfloor_{T / M}\right) A\right)^{-1}\left(\operatorname{Id}_{H}-\frac{T}{M} A\right)^{\left(\lfloor s\rfloor_{T / M}-\lfloor t\rfloor_{T / M}\right)^{\frac{M}{T}}}\right\|_{L\left(H, L^{q}\left(\lambda_{\mathcal{D}} ; \mathbb{R}\right)\right)} \\
& \leq \sup _{M \in \mathbb{N}} \sup _{t \in(0, T]} 2^{[\rho-1]^{+}} C\left(t-\lfloor t\rfloor_{T / M}\right)^{\rho}\left\|(-A)^{(d / 4-d / 2 q)}\left(\operatorname{Id}_{H}-\left(t-\lfloor t\rfloor_{T / M}\right) A\right)^{-1}\right\|_{L(H)} \\
& +\sup _{M \in \mathbb{N}} \sup _{l \in\{1,2, \ldots, M\}} \sup _{k \in\{0,1, \ldots, l-1\}} 2^{[\rho-1]^{+}} C\left(\frac{T}{M}(l-k)\right)^{\rho}\left\|(-A)^{(d / 4-d / 2 q)}\left(\operatorname{Id}_{H}-\frac{T}{M} A\right)^{-(l-k)}\right\|_{L(H)} \\
& \leq 2^{[\rho-1]^{+}} C \sup _{M \in \mathbb{N}}\left[\sup _{t \in(0, T]}\left(t-\lfloor t\rfloor_{T / M}\right)^{(\rho-[d / 4-d / 2 q])}+\left(\frac{T}{M}\right)^{\rho}\left\|(-A)^{(d / 4-d / 2 q)}\left(\operatorname{Id}_{H}-\frac{T}{M} A\right)^{-1}\right\|_{L(H)}\right] \\
& +2^{[\rho-1]^{+}} C \sup _{M \in\{2,3, \ldots\}} \sup _{l \in\{2,3, \ldots, M\}} \sup _{k \in\{0,1, \ldots, l-2\}}\left(\frac{T}{M}(l-k)\right)^{\rho}\left\|(-A)^{(d / 4-d / 2 q)}\left(\operatorname{Id}_{H}-\frac{T}{M} A\right)^{-(l-k)}\right\|_{L(H)} .
\end{aligned}
$$

This, Lemma 6.4, and again the fact that $\forall s \in[0, \infty), \kappa \in[0,1]:\left\|(-s A)^{\kappa}\left(\operatorname{Id}_{H}-s A\right)^{-1}\right\|_{L(H)} \leq 1$ 
prove that

$$
\begin{aligned}
& \sup _{M \in \mathbb{N}} \sup _{0 \leq s<t \leq T}(t-s)^{\rho}\left\|\left(\operatorname{Id}_{H}-\left(t-\lfloor t\rfloor_{T / M}\right) A\right)^{-1}\left(\operatorname{Id}_{H}-\frac{T}{M} A\right)^{\left(\lfloor s\rfloor_{T / M}-\lfloor t\rfloor_{T / M}\right) \frac{M}{T}}\right\|_{L\left(H, L^{q}\left(\lambda_{\mathcal{D}} ; \mathbb{R}\right)\right)} \\
& \leq 2^{[\rho-1]^{+}} C\left[\sup _{M \in \mathbb{N}} 2\left(\frac{T}{M}\right)^{(\rho-[d / 4-d / 2 q])}+\sup _{M \in\{2,3, \ldots\}} \sup _{l \in\{2,3, \ldots, M\}} \sup _{k \in\{0,1, \ldots, l-2\}} 4\left(\frac{T}{M}(l-k)\right)^{(\rho-[d / 4-d / 2 q])}\right] \\
& \leq 6 \cdot 2^{[\rho-1]^{+}} C T^{(\rho-[d / 4-d / 2 q])}<\infty .
\end{aligned}
$$

The proof of Lemma 6.5 is thus completed.

Lemma 6.6. Assume the setting in Section 6.1 and let $q \in\{2 n, 2 n+2,2 n+4, \ldots\}, v, w \in$ $L^{n q}\left(\lambda_{\mathcal{D}} ; \mathbb{R}\right)$. Then

$$
\|F(v+w)\|_{H} \leq 2^{(n+1)} \sqrt{\max \left\{1, \lambda_{\mathbb{R}^{d}}(\mathcal{D})\right\}}\left[\max _{k \in\{0,1, \ldots, n\}}\left|a_{k}\right|\right]\left(1+\|v\|_{L^{q}\left(\lambda_{\mathcal{D}} ; \mathbb{R}\right)}^{q / 2}+\|w\|_{L^{q}\left(\lambda_{\mathcal{D}} ; \mathbb{R}\right)}^{q / 2}\right) .
$$

Proof of Lemma 6.6. Note that Hölder's inequality ensures that

$$
\begin{aligned}
\|F(v+w)\|_{H} & \leq \sum_{k=0}^{n}\left|a_{k}\right|\left\|[v+w]^{k}\right\|_{H}=\left|a_{0}\right| \sqrt{\lambda_{\mathbb{R}^{d}}(\mathcal{D})}+\sum_{k=1}^{n}\left|a_{k}\right|\|v+w\|_{L^{2 k}\left(\lambda_{\mathcal{D}} ; \mathbb{R}\right)}^{k} \\
& \leq\left|a_{0}\right| \sqrt{\lambda_{\mathbb{R}^{d}}(\mathcal{D})}+\sum_{k=1}^{n}\left|a_{k}\right|\left[\left|\lambda_{\mathbb{R}^{d}}(\mathcal{D})\right|^{\left(\frac{1}{2 k}-\frac{1}{q}\right)}\|v+w\|_{L^{q}\left(\lambda_{\mathcal{D}} ; \mathbb{R}\right)}\right]^{k} \\
& \leq\left|a_{0}\right| \sqrt{\lambda_{\mathbb{R}^{d}}(\mathcal{D})}+\sum_{k=1}^{n}\left|a_{k}\right|\left|\lambda_{\mathbb{R}^{d}}(\mathcal{D})\right|^{\left(\frac{1}{2}-\frac{k}{q}\right)}\left[\|v\|_{L^{q}\left(\lambda_{\mathcal{D}} ; \mathbb{R}\right)}+\|w\|_{L^{q}\left(\lambda_{\mathcal{D}} ; \mathbb{R}\right)}\right]^{k} \\
& =\sum_{k=0}^{n}\left|a_{k}\right|\left|\lambda_{\mathbb{R}^{d}}(\mathcal{D})\right|^{\left(\frac{1}{2}-\frac{k}{q}\right)}\left[\|v\|_{L^{q}\left(\lambda_{\mathcal{D}} ; \mathbb{R}\right)}+\|w\|_{L^{q}\left(\lambda_{\mathcal{D}} ; \mathbb{R}\right)}\right]^{k} .
\end{aligned}
$$

The fact that $\forall x, y, r \in[0, \infty):(x+y)^{r} \leq 2^{[r-1]^{+}}\left(x^{r}+y^{r}\right)$ hence implies that

$$
\begin{aligned}
\|F(v+w)\|_{H} & \leq \sqrt{\max \left\{1, \lambda_{\mathbb{R}^{d}}(\mathcal{D})\right\}} \sum_{k=0}^{n}\left|a_{k}\right| 2^{(k-1)}\left[\|v\|_{L^{q}\left(\lambda_{\mathcal{D}} ; \mathbb{R}\right)}^{k}+\|w\|_{L^{q}\left(\lambda_{\mathcal{D}} ; \mathbb{R}\right)}^{k}\right] \\
& \leq \sqrt{\max \left\{1, \lambda_{\mathbb{R}^{d}}(\mathcal{D})\right\}} \sum_{k=0}^{n}\left|a_{k}\right| 2^{k}\left[1+\|v\|_{L^{q}\left(\lambda_{\mathcal{D}} ; \mathbb{R}\right)}^{q / 2}+\|w\|_{L^{q}\left(\lambda_{\mathcal{D}} ; \mathbb{R}\right)}^{q / 2}\right] \\
& \leq \sqrt{\max \left\{1, \lambda_{\mathbb{R}^{d}}(\mathcal{D})\right\}}\left[\max _{k \in\{0,1, \ldots, n\}}\left|a_{k}\right|\right]\left[\sum_{k=0}^{n} 2^{k}\right]\left[1+\|v\|_{L^{q}\left(\lambda_{\mathcal{D}} ; \mathbb{R}\right)}^{q / 2}+\|w\|_{L^{q}\left(\lambda_{\mathcal{D}} ; \mathbb{R}\right)}^{q / 2}\right] .
\end{aligned}
$$

This completes the proof of Lemma 6.6.

Lemma 6.7. Assume the setting in Section 6.1 and let $v, w \in V$ with $v-w \in H_{1}$. Then

$$
\begin{aligned}
& \langle v-w, A(v-w)+F(v)-F(w)\rangle_{H} \\
& \leq(n-1) \max \left\{1,\left|a_{n}\right|^{(2-n)}\right\} \max \left\{1, \max _{k \in\{0,1, \ldots, n-1\}}\left[k\left|a_{k}\right|\right]^{(n-1)}\right\}\|v-w\|_{H}^{2} .
\end{aligned}
$$


Proof of Lemma 6.7. Throughout this proof assume w.l.o.g. that $n>1$ (otherwise the proof is clear) and let $f: \mathbb{R} \rightarrow \mathbb{R}$ be the function with the property that for all $x \in \mathbb{R}$ it holds that $f(x)=\sum_{k=0}^{n} a_{k} x^{k}$. Note that the fundamental theorem of calculus implies that for all $x, y \in \mathbb{R}$ it holds that

$$
(x-y)(f(x)-f(y))=(x-y) \int_{y}^{x} f^{\prime}(z) d z \leq\left[\sup _{z \in \mathbb{R}} f^{\prime}(z)\right](x-y)^{2} .
$$

Next note that Young's inequality shows that for all $x \in \mathbb{R}$ it holds that

$$
\begin{aligned}
& f^{\prime}(x)=\sum_{k=1}^{n} k a_{k} x^{(k-1)} \leq n a_{n} x^{(n-1)}+\left|a_{1}\right|+\sum_{k=2}^{n-1} k\left|a_{k}\right||x|^{(k-1)} \\
& =n a_{n} x^{(n-1)}+\left|a_{1}\right|+\sum_{k=2}^{n-1} k\left|a_{k}\right|\left[\frac{(n-1)\left|a_{n}\right|}{(k-1) \max \left\{1, k\left|a_{k}\right|\right\}}\right]^{\frac{(k-1)}{(n-1)}}|x|^{(k-1)}\left[\frac{(n-1)\left|a_{n}\right|}{(k-1) \max \left\{1, k\left|a_{k}\right|\right\}}\right]^{-\frac{(k-1)}{(n-1)}} \\
& \leq n a_{n} x^{(n-1)}+\left|a_{1}\right|+\sum_{k=2}^{n-1} k\left|a_{k}\right|\left(\frac{\left|a_{n}\right| x^{(n-1)}}{\max \left\{1, k\left|a_{k}\right|\right\}}+\frac{(n-k)}{(n-1)}\left[\frac{(n-1)\left|a_{n}\right|}{(k-1) \max \left\{1, k\left|a_{k}\right|\right\}}\right]^{-\frac{(k-1)}{(n-k)}}\right) \\
& \leq\left[n a_{n}+(n-2)\left|a_{n}\right|\right] x^{(n-1)}+\left|a_{1}\right|+\sum_{k=2}^{n-1} k\left|a_{k}\right|\left[\frac{\max \left\{1, k\left|a_{k}\right|\right\}}{\left|a_{n}\right|}\right]^{(k-1)} \frac{(k-k)}{n-1} \\
& \leq \sum_{k=1}^{n} \max \left\{1,\left|a_{n}\right|^{-(n-2)}\right\}\left[\max \left\{1, k\left|a_{k}\right|\right\}\right]^{(n-1)} \\
& \leq(n-1) \max \left\{1,\left|a_{n}\right|^{-(n-2)}\right\} \max \left\{1, \max _{k \in\{0,1, \ldots, n-1\}}\left[k\left|a_{k}\right|\right]^{(n-1)}\right\} .
\end{aligned}
$$

In addition, observe that (127)-(128) prove that

$$
\begin{aligned}
& \langle v-w, F(v)-F(w)\rangle_{H}=\int_{\mathcal{D}}(v(x)-w(x))[f(v(x))-f(w(x))] \lambda_{\mathbb{R}^{d}}(d x) \\
& \leq(n-1) \max \left\{1,\left|a_{n}\right|^{-(n-2)}\right\} \max \left\{1, \max _{k \in\{0,1, \ldots, n-1\}}\left[k\left|a_{k}\right|\right]^{(n-1)}\right\} \int_{\mathcal{D}}(v(x)-w(x))^{2} \lambda_{\mathbb{R}^{d}}(d x) \\
& =(n-1) \max \left\{1,\left|a_{n}\right|^{-(n-2)}\right\} \max \left\{1, \max _{k \in\{0,1, \ldots, n-1\}}\left[k\left|a_{k}\right|\right]^{(n-1)}\right\}\|v-w\|_{H}^{2} .
\end{aligned}
$$

Furthermore, note that

$$
\begin{aligned}
\langle v-w, A(v-w)\rangle_{H} & =-\left\langle v-w,(-A)^{1 / 2}(-A)^{1 / 2}(v-w)\right\rangle_{H} \\
& =-\left\langle(-A)^{1 / 2}(v-w),(-A)^{1 / 2}(v-w)\right\rangle_{H}=-\left\|(-A)^{1 / 2}(v-w)\right\|_{H} \leq 0 .
\end{aligned}
$$

Combining this with (129) completes the proof of Lemma 6.7. 
Lemma 6.8. Assume the setting in Section 6.1 and let $v, w \in V$. Then

$$
\begin{aligned}
& \|F(v)-F(w)\|_{H}^{2} \\
& \leq \max \left\{1, \lambda_{\mathbb{R}^{d}}(\mathcal{D})\right\}\left[\frac{n(n+1)}{2} \max _{j \in\{1,2, \ldots, n\}}\left|a_{j}\right|\right]^{2}\|v-w\|_{V}^{2}\left(1+\|v\|_{V}^{\max \{1,2(n-1)\}}+\|w\|_{V}^{\max \{1,2(n-1)\}}\right) .
\end{aligned}
$$

Proof of Lemma 6.8. Throughout this proof assume w.l.o.g. that $n>1$ (otherwise the proof is clear). Next note that the fundamental theorem of calculus implies that for all $k \in \mathbb{N}, x, y \in \mathbb{R}$ it holds that

$$
\begin{aligned}
\left|x^{k}-y^{k}\right| & =\left|\int_{0}^{1} k(y+r(x-y))^{(k-1)}(x-y) d r\right| \leq k|x-y| \int_{0}^{1}|r x+(1-r) y|^{(k-1)} d r \\
& \leq k|x-y| \int_{0}^{1}\left(r|x|^{(k-1)}+(1-r)|y|^{(k-1)}\right) d r .
\end{aligned}
$$

This and Hölder's inequality prove that

$$
\begin{aligned}
& \|F(v)-F(w)\|_{H}=\left\|\sum_{k=0}^{n} a_{k}\left(v^{k}-w^{k}\right)\right\|_{H} \leq \sum_{k=1}^{n}\left|a_{k}\right|\left\|v^{k}-w^{k}\right\|_{H} \\
& \leq \sum_{k=1}^{n} k\left|a_{k}\right| \int_{0}^{1}\left\||v-w|\left(r|v|^{(k-1)}+(1-r)|w|^{(k-1)}\right)\right\|_{H} d r \\
& \leq \sum_{k=1}^{n} k\left|a_{k}\right| \int_{0}^{1}\|v-w\|_{V}\left\|r|v|^{(k-1)}+(1-r)|w|^{(k-1)}\right\|_{L^{2 n^{2} /\left(n^{2}-1\right)}\left(\lambda_{\mathcal{D}} ; \mathbb{R}\right)} d r \\
& \leq\|v-w\|_{V}\left[\left|a_{1}\right| \sqrt{\max \left\{1, \lambda_{\mathbb{R}^{d}}(\mathcal{D})\right\}}\right. \\
& \left.+\sum_{k=2}^{n} k\left|a_{k}\right| \int_{0}^{1}\left(r\|v\|_{L^{2 n^{2}(k-1) /\left(n^{2}-1\right)\left(\lambda_{\mathcal{D}} ; \mathbb{R}\right)}}^{(k-1)}+(1-r)\|w\|_{L^{2 n^{2}(k-1) /\left(n^{2}-1\right)\left(\lambda_{\mathcal{D}} ; \mathbb{R}\right)}}^{(k-1)}\right) d r\right] \text {. }
\end{aligned}
$$


Again Hölder's inequality hence ensures that

$$
\begin{aligned}
& \|F(v)-F(w)\|_{H} \\
& \leq\|v-w\|_{V}\left[\left|a_{1}\right| \sqrt{\max \left\{1, \lambda_{\mathbb{R}^{d}}(\mathcal{D})\right\}}\right. \\
& \left.+\frac{1}{2} \sum_{k=2}^{n} k\left|a_{k}\right|\left(\|v\|_{L^{2 n^{2}(k-1) /\left(n^{2}-1\right)\left(\lambda_{\mathcal{D}} ; \mathbb{R}\right)}}^{(k-1)}+\|w\|_{L^{2 n^{2}(k-1) /\left(n^{2}-1\right)\left(\lambda_{\mathcal{D}} ; \mathbb{R}\right)}}^{(k-1)}\right)\right] \\
& \leq\|v-w\|_{V}\left[\left|a_{1}\right| \sqrt{\max \left\{1, \lambda_{\mathbb{R}^{d}}(\mathcal{D})\right\}}+\frac{1}{2} \sum_{k=2}^{n} k\left|a_{k}\right|\left|\lambda_{\mathbb{R}^{d}}(\mathcal{D})\right|^{\frac{\left(n^{2}-k\right)}{2 n^{2}}}\left(\|v\|_{V}^{(k-1)}+\|w\|_{V}^{(k-1)}\right)\right] \\
& \leq \frac{1}{2}\|v-w\|_{V} \sqrt{\max \left\{1, \lambda_{\mathbb{R}^{d}}(\mathcal{D})\right\}}\left[\max _{j \in\{1,2, \ldots, n\}}\left|a_{j}\right|\right] \sum_{k=1}^{n} k\left(\|v\|_{V}^{(k-1)}+\|w\|_{V}^{(k-1)}\right) \\
& \leq \frac{n(n+1)}{4}\|v-w\|_{V} \sqrt{\max \left\{1, \lambda_{\mathbb{R}^{d}}(\mathcal{D})\right\}}\left[\max _{j \in\{1,2, \ldots, n\}}\left|a_{j}\right|\right]\left(\max \left\{1,\|v\|_{V}^{(n-1)}\right\}+\max \left\{1,\|w\|_{V}^{(n-1)}\right\}\right) \text {. }
\end{aligned}
$$

Combining this with the fact that $\forall x, y \in \mathbb{R}:|x+y|^{2} \leq 2\left(x^{2}+y^{2}\right)$ completes the proof of Lemma 6.8,

Lemma 6.9. Assume the setting in Section 6.1 and let $q \in[2, \infty), \varrho \in[0, \infty) \cap(-\infty, 1+d / 2 q-d / 4)$. Then $\sup _{M \in \mathbb{N}} \sup _{t \in(0, T]} M^{\varrho} \int_{0}^{t}\left\|e^{(t-s) A}-e^{\left(t-\lfloor s\rfloor_{T / M}\right) A}\right\|_{L\left(H, L^{q}\left(\lambda_{\mathcal{D}} ; \mathbb{R}\right)\right)} d s<\infty$.

Proof of Lemma 6.9. Observe that for all $t \in(0, T], M \in \mathbb{N}$ it holds that

$$
\begin{aligned}
& \int_{0}^{t}\left\|e^{(t-s) A}-e^{\left(t-\lfloor s\rfloor_{T / M}\right) A}\right\|_{L\left(H, L^{q}\left(\lambda_{\mathcal{D}} ; \mathbb{R}\right)\right)} d s \\
& =\int_{0}^{t}\left\|e^{(t-s) A}\left(\operatorname{Id}_{H}-e^{\left(s-\lfloor s\rfloor_{T / M}\right) A}\right)\right\|_{L\left(H, L^{q}\left(\lambda_{\mathcal{D}} ; \mathbb{R}\right)\right)} d s \\
& \leq \int_{0}^{t}\left\|e^{\frac{1}{2}(t-s) A}\right\|_{L\left(H, L^{q}\left(\lambda_{\mathcal{D}} ; \mathbb{R}\right)\right)}\left\|e^{\frac{1}{2}(t-s) A}\left(\operatorname{Id}_{H}-e^{\left(s-\lfloor s\rfloor_{T / M}\right) A}\right)\right\|_{L(H)} d s \\
& \leq\left[\sup _{s \in(0, T]} s^{(d / 4-d / 2 q)}\left\|e^{s A}\right\|_{L\left(H, L^{q}\left(\lambda_{\mathcal{D}} ; \mathbb{R}\right)\right)}\right] \int_{0}^{t}\left[\frac{1}{2}(t-s)\right]^{-(\varrho+d / 4-d / 2 q)}\left(s-\lfloor s\rfloor_{T / M}\right)^{\varrho} \\
& \quad \cdot\left\|\left(-\frac{1}{2}(t-s) A\right)^{\varrho} e^{\frac{1}{2}(t-s) A}\right\|_{L(H)}\left\|\left(-\left(s-\lfloor s\rfloor_{T / M}\right) A\right)^{-\varrho}\left(\operatorname{Id}_{H}-e^{\left(s-\lfloor s\rfloor_{T / M}\right) A}\right)\right\|_{L(H)} d s .
\end{aligned}
$$

This and the facts that $\forall s \in[0, \infty), r \in[0,1]:\left\|(-s A)^{r} e^{s A}\right\|_{L(H)} \leq 1$ and $\forall s \in(0, \infty), r \in$ $[0,1]:\left\|(-s A)^{-r}\left(\operatorname{Id}_{H}-e^{s A}\right)\right\|_{L(H)} \leq 1$ show that for all $t \in(0, T], M \in \mathbb{N}$ it holds that

$$
\begin{aligned}
& \int_{0}^{t}\left\|e^{(t-s) A}-e^{\left(t-\lfloor s\rfloor_{T / M}\right) A}\right\|_{L\left(H, L^{q}\left(\lambda_{\mathcal{D}} ; \mathbb{R}\right)\right)} d s \\
& \leq\left[\sup _{s \in(0, T]} s^{(d / 4-d / 2 q)}\left\|e^{s A}\right\|_{L\left(H, L^{q}(\mathcal{D} ; \mathbb{R})\right)}\right] \int_{0}^{t}\left[\frac{1}{2}(t-s)\right]^{-(\varrho+d / 4-d / 2 q)} \frac{T^{\varrho}}{M^{\varrho}} d s \\
& \leq \frac{2 T^{(1+d / 2 q-d / 4)}}{(1-\varrho-d / 4+d / 2 q) M^{\varrho}}\left[\sup _{s \in(0, T]} s^{(d / 4-d / 2 q)}\left\|e^{s A}\right\|_{L\left(H, L^{q}\left(\lambda_{\mathcal{D}} ; \mathbb{R}\right)\right)}\right] .
\end{aligned}
$$


Combining this with Lemma 6.5 completes the proof of Lemma 6.9.

Lemma 6.10. Assume the setting in Section 6.1 and let $t \in(0, \infty), \kappa \in[0,2]$. Then

$$
\left\|(-t A)^{-\kappa}\left(e^{t A}-\left(\operatorname{Id}_{H}-t A\right)^{-1}\right)\right\|_{L(H)} \leq 1 .
$$

Proof of Lemma 6.10. Observe that the fundamental theorem of calculus shows that for all $v \in H$, $r \in[0,1]$ it holds that

$$
\begin{aligned}
& \left\|(-t A)^{-r}\left(e^{t A}-\left(\operatorname{Id}_{H}-t A\right)^{-1}\right) v\right\|_{H} \\
& =\left\|(-t A)^{-r}\left(\operatorname{Id}_{H}-t A\right)^{-1}\left(e^{t A}-t A e^{t A}-\operatorname{Id}_{H}\right) v\right\|_{H} \\
& =\left\|(-t A)^{-r}\left(\operatorname{Id}_{H}-t A\right)^{-1}\left(\operatorname{Id}_{H}+\int_{0}^{t} A e^{u A} d u-t A e^{t A}-\operatorname{Id}_{H}\right) v\right\|_{H} \\
& =\left\|(-t A)^{-r}\left(\operatorname{Id}_{H}-t A\right)^{-1}\left(\int_{0}^{t} A\left(e^{u A}-e^{t A}\right) d u\right) v\right\|_{H} .
\end{aligned}
$$

This and the facts that $\forall s \in(0, \infty), r \in[0,1]:\left\|(-s A)^{-r}\left(\operatorname{Id}_{H}-e^{s A}\right)\right\|_{L(H)} \leq 1, \forall s \in[0, \infty)$, $r \in[0,1]:\left\|(-s A)^{r} e^{s A}\right\|_{L(H)} \leq 1$, and $\forall s \in[0, \infty), r \in[0,1]:\left\|(-s A)^{r}\left(\operatorname{Id}_{H}-s A\right)^{-1}\right\|_{L(H)} \leq 1$ imply that for all $v \in H, r \in[0,1]$ it holds that

$$
\begin{aligned}
& \left\|(-t A)^{-r}\left(e^{t A}-\left(\operatorname{Id}_{H}-t A\right)^{-1}\right) v\right\|_{H} \\
& \leq\left\|(-t A)^{(1-r)}\left(\operatorname{Id}_{H}-t A\right)^{-1}\right\|_{L(H)}\left\|t^{-1}\left(\int_{0}^{t} e^{u A}\left(\operatorname{Id}_{H}-e^{(t-u) A}\right) d u\right) v\right\|_{H} \\
& \leq t^{-1} \int_{0}^{t}\left\|e^{u A}\right\|_{L(H)}\left\|\operatorname{Id}_{H}-e^{(t-u) A}\right\|_{L(H)}\|v\|_{H} d u \leq\|v\|_{H} .
\end{aligned}
$$

Moreover, note that a Taylor expansion proves that for all $v \in H, r \in(1,2]$ it holds that

$$
\begin{aligned}
& \left\|(-t A)^{-r}\left(e^{t A}-\left(\operatorname{Id}_{H}-t A\right)^{-1}\right) v\right\|_{H} \\
& =\left\|(-t A)^{-r}\left(\operatorname{Id}_{H}-t A\right)^{-1}\left(e^{t A}-t A e^{t A}-\operatorname{Id}_{H}\right) v\right\|_{H} \\
& =\left\|(-t A)^{-r}\left(\operatorname{Id}_{H}-t A\right)^{-1}\left(t A+\int_{0}^{t} A^{2} e^{u A}(t-u) d u-t A\left(\operatorname{Id}_{H}+\int_{0}^{t} A e^{u A} d u\right)\right) v\right\|_{H} \\
& =\left\|(-t A)^{-r}\left(\operatorname{Id}_{H}-t A\right)^{-1}\left(-\int_{0}^{t} A^{2} e^{u A} u d u\right) v\right\|_{H} .
\end{aligned}
$$

The facts that $\forall s \in[0, \infty), r \in[0,1]:\left\|(-s A)^{r}\left(\operatorname{Id}_{H}-s A\right)^{-1}\right\|_{L(H)} \leq 1$ and $\forall s \in[0, \infty), r \in$ $[0,1]:\left\|(-s A)^{r} e^{s A}\right\|_{L(H)} \leq 1$ hence yield that for all $v \in H, r \in(1,2]$ it holds that

$$
\begin{aligned}
& \left\|(-t A)^{-r}\left(e^{t A}-\left(\operatorname{Id}_{H}-t A\right)^{-1}\right) v\right\|_{H} \\
& \leq\left\|(-t A)^{(2-r)}\left(\operatorname{Id}_{H}-t A\right)^{-1}\right\|_{L(H)}\left\|t^{-2}\left(\int_{0}^{t} e^{u A} u d u\right) v\right\|_{H} \\
& \leq t^{-2} \int_{0}^{t} u\left\|e^{u A}\right\|_{L(H)}\|v\|_{H} d u \leq \frac{1}{2}\|v\|_{H} .
\end{aligned}
$$

Combining (139) and (141) completes the proof of Lemma 6.10. 
Lemma 6.11. Assume the setting in Section 6.1 and let $t \in(0, \infty), \kappa \in[0,1], m \in \mathbb{N}$. Then

$$
\left\|(-t A)^{\kappa}\left(e^{m t A}-\left(\operatorname{Id}_{H}-t A\right)^{-m}\right)\right\|_{L(H)} \leq \frac{16}{m} \text {. }
$$

Proof of Lemma 6.11. First of all, note that the triangle inequality and the facts that $\forall s \in[0, \infty)$, $r \in[0,1]:\left\|(-s A)^{r} e^{s A}\right\|_{L(H)} \leq 1$ and $\forall s \in[0, \infty), r \in[0,1]:\left\|(-s A)^{r}\left(\operatorname{Id}_{H}-s A\right)^{-1}\right\|_{L(H)} \leq 1$ imply that

$$
\left\|(-t A)^{\kappa}\left(e^{t A}-\left(\operatorname{Id}_{H}-t A\right)^{-1}\right)\right\|_{L(H)} \leq\left\|(-t A)^{\kappa} e^{t A}\right\|_{L(H)}+\left\|(-t A)^{\kappa}\left(\operatorname{Id}_{H}-t A\right)^{-1}\right\|_{L(H)} \leq 2 .
$$

and

$$
\left\|(-t A)^{\kappa}\left(e^{t A}+\left(\operatorname{Id}_{H}-t A\right)^{-1}\right)\right\|_{L(H)} \leq\left\|(-t A)^{\kappa} e^{t A}\right\|_{L(H)}+\left\|(-t A)^{\kappa}\left(\operatorname{Id}_{H}-t A\right)^{-1}\right\|_{L(H)} \leq 2 .
$$

Next observe that Lemma 6.10 and (144) prove that

$$
\begin{aligned}
& \left\|(-t A)^{\kappa}\left(e^{2 t A}-\left(\operatorname{Id}_{H}-t A\right)^{-2}\right)\right\|_{L(H)} \\
& =\left\|(-t A)^{\kappa}\left(e^{t A}+\left(\operatorname{Id}_{H}-t A\right)^{-1}\right)\left(e^{t A}-\left(\operatorname{Id}_{H}-t A\right)^{-1}\right)\right\|_{L(H)} \\
& \leq\left\|(-t A)^{\kappa}\left(e^{t A}+\left(\operatorname{Id}_{H}-t A\right)^{-1}\right)\right\|_{L(H)}\left\|e^{t A}-\left(\operatorname{Id}_{H}-t A\right)^{-1}\right\|_{L(H)} \leq 2 .
\end{aligned}
$$

Moreover, note that Lemma 6.10 ensures that for all $j \in\{3,4, \ldots\}$ it holds that

$$
\begin{aligned}
& \left\|(-t A)^{\kappa}\left(e^{j t A}-\left(\operatorname{Id}_{H}-t A\right)^{-j}\right)\right\|_{L(H)} \\
& =\left\|\sum_{l=0}^{j-1}(-t A)^{\kappa} e^{l t A}\left(\operatorname{Id}_{H}-t A\right)^{(l-j+1)}\left(e^{t A}-\left(\operatorname{Id}_{H}-t A\right)^{-1}\right)\right\|_{L(H)} \\
& \leq t^{\kappa} \sum_{l=0}^{j-1}\left\|(-A)^{2} e^{l t A}\left(\operatorname{Id}_{H}-t A\right)^{(l-j+1)}\right\|_{L(H)}\left\|(-A)^{-(2-\kappa)}\left(e^{t A}-\left(\operatorname{Id}_{H}-t A\right)^{-1}\right)\right\|_{L(H)} \\
& \leq t^{2} \sum_{l=0}^{j-1}\left\|(-A)^{2} e^{l t A}\left(\operatorname{Id}_{H}-t A\right)^{(l-j+1)}\right\|_{L(H)} .
\end{aligned}
$$

Lemma 6.4 and the fact that $\forall s \in[0, \infty), r \in[0,2]:\left\|(-s A)^{r} e^{s A}\right\|_{L(H)} \leq 1$ therefore yield that for all $j \in\{3,4, \ldots\}$ it holds that

$$
\begin{aligned}
& \left\|(-t A)^{\kappa}\left(e^{j t A}-\left(\operatorname{Id}_{H}-t A\right)^{-j}\right)\right\|_{L(H)} \\
& \leq t^{2}\left[\sum_{l=0}^{\lceil j / 2\rfloor_{1}-1}\left\|(-A)^{2}\left(\operatorname{Id}_{H}-t A\right)^{(l-j+1)}\right\|_{L(H)}+\sum_{l=\lfloor j / 2\rfloor_{1}}^{j-1}\left\|(-A)^{2} e^{l t A}\right\|_{L(H)}\right] \\
& \leq \sum_{l=0}^{\lfloor j / 2\rfloor_{1}-1} \frac{4 t^{2}}{t^{2}(j-l-1)^{2}}+\sum_{l=\lfloor j / 2\rfloor_{1}}^{j-1} \frac{t^{2}}{t^{2} l^{2}} \leq \sum_{l=0}^{\lfloor j / 2\rfloor_{1}-1} \frac{4}{\left(j-\lfloor j / 2\rfloor_{1}\right)^{2}}+\sum_{l=\lfloor j / 2\rfloor_{1}}^{j-1} \frac{1}{\left|\lfloor j / 2\rfloor_{1}\right|^{2}} \\
& =\frac{4\lfloor j / 2\rfloor_{1}}{\left|\lceil j / 2\rceil_{1}\right|^{2}}+\frac{\left(j-\lfloor j / 2\rfloor_{1}\right)}{\left|\lfloor j / 2\rfloor_{1}\right|^{2}} \leq \frac{4}{\lceil j / 2\rceil_{1}}+\frac{4}{\lceil j / 2\rceil_{1}} \leq \frac{16}{j} .
\end{aligned}
$$

Combining (143), (145), and (147) completes the proof of Lemma 6.11. 
Lemma 6.12. Assume the setting in Section 6.1 and let $q \in[2, \infty), \varrho \in[0, \infty) \cap(-\infty, 1+d / 2 q-d / 4)$. Then

$$
\sup _{M \in \mathbb{N}} \sup _{t \in(0, T]} M^{\varrho} \int_{0}^{t}\left\|e^{(t-s) A}-\left(\operatorname{Id}_{H}-\left(t-\lfloor t\rfloor_{T / M}\right) A\right)^{-1}\left(\operatorname{Id}_{H}-\frac{T}{M} A\right)^{\left(\lfloor s\rfloor_{T / M}-\lfloor t\rfloor_{T / M}\right) \frac{M}{T}}\right\|_{L\left(H, L^{q}\left(\lambda_{\mathcal{D}} ; \mathbb{R}\right)\right)} d s
$$

Proof of Lemma 6.12. Throughout this proof let $R:[0, T] \rightarrow L(H)$ be the function with the property that for all $t \in[0, T]$ it holds that $R_{t}=\left(\operatorname{Id}_{H}-t A\right)^{-1}$. Next note that the triangle inequality implies that for all $M \in \mathbb{N}, t \in(0, T]$ it holds that

$$
\begin{aligned}
& \int_{0}^{t}\left\|e^{(t-s) A}-R_{\left(t-\lfloor t\rfloor_{T / M}\right)}\left[R_{T / M}\right]^{\left(\lfloor t\rfloor_{T / M}-\lfloor s\rfloor_{T / M}\right) \frac{M}{T}}\right\|_{L\left(H, L^{q}\left(\lambda_{\mathcal{D}} ; \mathbb{R}\right)\right)} d s \\
& \leq \int_{0}^{t}\left\|e^{(t-s) A}-e^{\left(t-\lfloor s\rfloor_{T / M}\right) A}\right\|_{L\left(H, L^{q}\left(\lambda_{\mathcal{D}} ; \mathbb{R}\right)\right)} d s \\
& +\int_{0}^{t}\left\|e^{\left(t-\lfloor s\rfloor_{T / M}\right) A}-R_{\left(t-\lfloor t\rfloor_{T / M}\right)}\left[R_{T / M}\right]^{\left(\lfloor t\rfloor_{T / M}-\lfloor s\rfloor_{T / M}\right) \frac{M}{T}}\right\|_{L\left(H, L^{q}\left(\lambda_{\mathcal{D}} ; \mathbb{R}\right)\right)} d s \\
& \leq \int_{0}^{t}\left\|e^{(t-s) A}-e^{\left(t-\lfloor s\rfloor_{T / M}\right) A}\right\|_{L\left(H, L^{q}\left(\lambda_{\mathcal{D}} ; \mathbb{R}\right)\right)} d s \\
& +\int_{\max \left\{0,\lfloor t\rfloor_{T / M}-\frac{T}{M}\right\}}^{t}\left\|e^{\left(t-\lfloor s\rfloor_{T / M}\right) A}-R_{\left(t-\lfloor t\rfloor_{T / M}\right)}\left[R_{T / M}\right]^{\left(\lfloor t\rfloor_{T / M}-\lfloor s\rfloor_{T / M}\right) \frac{M}{T}}\right\|_{L\left(H, L^{q}\left(\lambda_{\mathcal{D}} ; \mathbb{R}\right)\right)} d s \\
& +\int_{0}^{\max \left\{0,\lfloor t\rfloor_{T / M}-\frac{T}{M}\right\}}\left\|e^{\left(\lfloor t\rfloor_{T / M}-\lfloor s\rfloor_{T / M}\right) A}\left(e^{\left(t-\lfloor t\rfloor_{T / M}\right) A}-R_{\left(t-\lfloor t\rfloor_{T / M}\right)}\right)\right\|_{L\left(H, L^{q}\left(\lambda_{\mathcal{D}} ; \mathbb{R}\right)\right)} d s \\
& +\int_{0}^{\max \left\{0,\lfloor t\rfloor_{T / M}-\frac{T}{M}\right\}}\left\|R_{\left(t-\lfloor t\rfloor_{T / M}\right)}\left(e^{\left(\lfloor t\rfloor_{T / M}-\lfloor s\rfloor_{T / M}\right) A}-\left[R_{T / M}\right]^{\left(\lfloor t\rfloor_{T / M}-\lfloor s\rfloor_{T / M}\right) \frac{M}{T}}\right)\right\|_{L\left(H, L^{q}\left(\lambda_{\mathcal{D}} ; \mathbb{R}\right)\right)} d s .
\end{aligned}
$$

Moreover, the triangle inequality and Lemma 6.5 prove that

$$
\begin{aligned}
& \sup _{M \in \mathbb{N}} \sup _{t \in(0, T]} M^{\varrho} \int_{\max \left\{0,\lfloor t\rfloor_{T / M}-\frac{T}{M}\right\}}^{t}\left\|e^{\left(t-\lfloor s\rfloor_{T / M}\right) A}-R_{\left(t-\lfloor t\rfloor_{T / M}\right)}\left[R_{T / M}\right]^{\left(\lfloor t\rfloor_{T / M}-\lfloor s\rfloor_{T / M}\right) \frac{M}{T}}\right\|_{L\left(H, L^{q}\left(\lambda_{\mathcal{D}} ; \mathbb{R}\right)\right)} d s \\
& \leq\left[\sup _{M \in \mathbb{N}} \sup _{t \in(0, T]} M^{\varrho} \int_{\max \left\{0,\lfloor t\rfloor_{T / M}-\frac{T}{M}\right\}}^{t}(t-s)^{-(d / 4-d / 2 q)} d s\right] \\
& \quad \cdot\left[\sup _{M \in \mathbb{N}} \sup _{0<s<t \leq T}(t-s)^{(d / 4-d / 2 q)}\left\|e^{\left(t-\lfloor s\rfloor_{T / M}\right) A}-R_{\left(t-\lfloor t\rfloor_{T / M}\right)}\left[R_{T / M}\right]^{\left(\lfloor t\rfloor_{T / M}-\lfloor s\rfloor_{T / M}\right) \frac{M}{T}}\right\|_{L\left(H, L^{q}\left(\lambda_{\mathcal{D}} ; \mathbb{R}\right)\right)}\right] \\
& \leq\left[\sup _{M \in \mathbb{N}} \frac{2 T^{(1+d / 2 q-d / 4)} M^{\varrho}}{(1+d / 2 q-d / 4) M^{(1+d / 2 q-d / 4)}}\right]\left[\sup _{s \in(0, T]} s^{(d / 4-d / 2 q)}\left\|e^{s A}\right\|_{L\left(H, L^{q}\left(\lambda_{\mathcal{D}} ; \mathbb{R}\right)\right)}\right]<\infty . \\
& \left.\quad+\sup _{M \in \mathbb{N}} \sup _{0<s<t \leq T}(t-s)^{(d / 4-d / 2 q)}\left\|R_{\left(t-\lfloor t\rfloor_{T / M}\right)}\left[R_{T / M}\right]^{\left(\lfloor t\rfloor_{T / M}-\lfloor s\rfloor_{T / M}\right) \frac{M}{T}}\right\|_{L\left(H, L^{q}\left(\lambda_{\mathcal{D}} ; \mathbb{R}\right)\right)}\right]<\infty .
\end{aligned}
$$


In the next step observe that the fact that $\forall s \in(0, \infty), r \in[0,1]:\left\|(-s A)^{r} e^{s A}\right\|_{L(H)} \leq 1$ and Lemma 6.10 assure that for all $t \in(0, T], M \in \mathbb{N}$ it holds that

$$
\begin{aligned}
& \int_{0}^{\max \left\{0,\lfloor t\rfloor_{T / M}-\frac{T}{M}\right\}}\left\|e^{\left(\lfloor t\rfloor_{T / M}-\lfloor s\rfloor_{T / M}\right) A}\left(e^{\left(t-\lfloor t\rfloor_{T / M}\right) A}-R_{\left(t-\lfloor t\rfloor_{T / M}\right)}\right)\right\|_{L\left(H, L^{q}\left(\lambda_{\mathcal{D}} ; \mathbb{R}\right)\right)} d s \\
& \leq \int_{0}^{\max \left\{0,\lfloor t\rfloor_{T / M}-\frac{T}{M}\right\}}\left\|e^{\frac{1}{2}\left(\lfloor t\rfloor_{T / M}-\lfloor s\rfloor_{T / M}\right) A}\right\|_{L\left(H, L^{q}\left(\lambda_{\mathcal{D}} ; \mathbb{R}\right)\right)} \\
& \quad \cdot\left\|e^{\frac{1}{2}\left(\lfloor t\rfloor_{T / M}-\lfloor s\rfloor_{T / M}\right) A}\left(e^{\left(t-\lfloor t\rfloor_{T / M}\right) A}-R_{\left(t-\lfloor t\rfloor_{T / M}\right)}\right)\right\|_{L(H)} d s \\
& \leq\left[\sup _{s \in(0, T]} s^{(d / 4-d / 2 q)}\left\|e^{s A}\right\|_{L\left(H, L^{q}\left(\lambda_{\mathcal{D}} ; \mathbb{R}\right)\right)}\right] \int_{0}^{\max \left\{0,\lfloor t\rfloor_{T / M}-\frac{T}{M}\right\}}\left[\frac{1}{2}\left(\lfloor t\rfloor_{T / M}-\lfloor s\rfloor_{T / M}\right)\right]^{-(d / 4-d / 2 q)} \\
& \cdot\left\|(-A) e^{\frac{1}{2}\left(\lfloor t\rfloor_{T / M}-\lfloor s\rfloor_{T / M}\right) A}\right\|_{L(H)}\left\|(-A)^{-1}\left(e^{\left(t-\lfloor t\rfloor_{T / M}\right) A}-R_{\left(t-\lfloor t\rfloor_{T / M}\right)}\right)\right\|_{L(H)} d s \\
& \leq \frac{T}{M}\left[\sup _{s \in(0, T]} s^{(d / 4-d / 2 q)}\left\|e^{s A}\right\|_{L\left(H, L^{q}\left(\lambda_{\mathcal{D}} ; \mathbb{R}\right)\right)}\right] \int_{0}^{\max \left\{0,\lfloor t\rfloor_{T / M}-\frac{T}{M}\right\}}\left[\frac{1}{2}\left(\lfloor t\rfloor_{T / M}-\lfloor s\rfloor_{T / M}\right)\right]^{-(1+d / 4-d / 2 q)} d s .
\end{aligned}
$$

Lemma 6.5 and the fact that $\forall M \in \mathbb{N}, \varepsilon \in(0, \infty): \sum_{l=2}^{M} l^{-(1+d / 4-d / 2 q+\varepsilon)} \leq \int_{1}^{M} s^{-(1+d / 4-d / 2 q+\varepsilon)} d s=$ $\frac{1-M^{-(d / 4-d / 2 q+\varepsilon)}}{d / 4-d / 2 q+\varepsilon}$ hence imply that for all $\varepsilon \in(0,1+d / 2 q-d / 4-\varrho]$ it holds that

$$
\begin{aligned}
& \sup _{M \in \mathbb{N}} \sup _{t \in(0, T]} M^{\varrho} \int_{0}^{\max \left\{0,\lfloor t\rfloor_{T / M}-\frac{T}{M}\right\}}\left\|e^{\left(\lfloor t\rfloor_{T / M}-\lfloor s\rfloor_{T / M}\right) A}\left(e^{\left(t-\lfloor t\rfloor_{T / M}\right) A}-R_{\left(t-\lfloor t\rfloor_{T / M}\right)}\right)\right\|_{L\left(H, L^{q}\left(\lambda_{\mathcal{D}} ; \mathbb{R}\right)\right)} d s \\
& \leq\left[\sup _{s \in(0, T]} s^{(d / 4-d / 2 q)}\left\|e^{s A}\right\|_{L\left(H, L^{q}\left(\lambda_{\mathcal{D}} ; \mathbb{R}\right)\right)}\right] \\
& \cdot\left[\sup _{M \in \mathbb{N}} \sup _{t \in(0, T]} \frac{4 T}{M^{(1-\varrho)}} \sum_{l=0}^{\lfloor t\rfloor_{T / M} \frac{M}{T}-2} \int_{\frac{l T}{M}}^{\frac{(l+1) T}{M}}\left[\left(\lfloor t\rfloor_{T / M} \frac{M}{T}-l\right) \frac{T}{M}\right]^{-(1+d / 4-d / 2 q)} d s\right] \\
& \leq\left[\sup _{s \in(0, T]} s^{(d / 4-d / 2 q)}\left\|e^{s A}\right\|_{L\left(H, L^{q}\left(\lambda_{\mathcal{D}} ; \mathbb{R}\right)\right)}\right]\left[\sup _{M \in \mathbb{N}} \sup _{t \in(0, T]} \frac{4 T^{(1-d / 4+d / 2 q)}}{M^{(1-\varrho-d / 4+d / 2 q)}} \sum_{l=2}^{\lfloor t\rfloor_{T / M} \frac{M}{T}} \frac{l^{\varepsilon}}{l^{(1+d / 4-d / 2 q+\varepsilon)}}\right] \\
& \leq\left[\sup _{s \in(0, T]} s^{(d / 4-d / 2 q)}\left\|e^{s A}\right\|_{L\left(H, L^{q}\left(\lambda_{\mathcal{D}} ; \mathbb{R}\right)\right)}\right]\left[\sup _{M \in \mathbb{N}} \frac{4 T^{(1-d / 4+d / 2 q)}\left(1-M^{-(d / 4-d / 2 q+\varepsilon)}\right)}{M^{(1-\varrho-d / 4+d / 2 q-\varepsilon)}(d / 4-d / 2 q+\varepsilon)}\right]<\infty .
\end{aligned}
$$

Furthermore, observe that the fact that for all $r \in[0, \infty)$ it holds that $H_{r} \subseteq W^{2 r, 2}(\mathcal{D}, \mathbb{R})$ continuously (cf., e.g., (A.46) in Da Prato \& Zabczyk [8] and Lunardi [33]) and the fact that for all $r \in[d / 4-d / 2 q, \infty)$ it holds that $W^{2 r, 2}(\mathcal{D}, \mathbb{R}) \subseteq W^{0, q}(\mathcal{D}, \mathbb{R})=L^{q}\left(\lambda_{\mathcal{D}} ; \mathbb{R}\right)$ continuously imply that there exists a real number $C \in(0, \infty)$ such that for all $v \in H_{(d / 4-d / 2 q)}$ it holds that $\|v\|_{L^{q}\left(\lambda_{\mathcal{D}} ; \mathbb{R}\right)} \leq C\|v\|_{H_{(d / 4-d / 2 q)}}$. This and the fact that $\forall s \in(0, \infty), r \in[0,1]:\left\|(-s A)^{r} R_{s}\right\|_{L(H)} \leq 1$ 
ensure that for all $M \in \mathbb{N}, s, t \in(0, T]$ with $s<\lfloor t\rfloor_{T / M}-T / M$ it holds that

$$
\begin{aligned}
& \sup _{v \in H \backslash\{0\}}\left[\frac{1}{\|v\|_{H}}\left\|R_{\left(t-\lfloor t\rfloor_{T / M}\right)}\left(e^{\left(\lfloor t\rfloor_{T / M}-\lfloor s\rfloor_{T / M}\right) A}-\left[R_{T / M}\right]^{\left(\lfloor t\rfloor_{T / M}-\lfloor s\rfloor_{T / M}\right) \frac{M}{T}}\right) v\right\|_{L^{q}\left(\lambda_{\mathcal{D}} ; \mathbb{R}\right)}\right] \\
& \leq \sup _{v \in H \backslash\{0\}}\left[\frac{C}{\|v\|_{H}}\left\|R_{\left(t-\lfloor t\rfloor_{T / M}\right)}\left(e^{\left(\lfloor t\rfloor_{T / M}-\lfloor s\rfloor_{T / M}\right) A}-\left[R_{T / M}\right]^{\left(\lfloor t\rfloor_{T / M}-\lfloor s\rfloor_{T / M}\right) \frac{M}{T}}\right) v\right\|_{H_{(d / 4-d / 2 q)}}\right] \\
& \leq \sup _{v \in H \backslash\{0\}}\left[\frac{C\left\|R_{\left(t-\lfloor t\rfloor_{T / M}\right)}\right\|_{L(H)}}{\|v\|_{H}}\left\|(-A)^{(d / 4-d / 2 q)}\left(e^{\left(\lfloor t\rfloor_{T / M}-\lfloor s\rfloor_{T / M}\right) A}-\left[R_{T / M}\right]^{\left(\lfloor t\rfloor_{T / M}-\lfloor s\rfloor_{T / M}\right) \frac{M}{T}}\right) v\right\|_{H}\right] \\
& \leq C\left\|(-A)^{(d / 4-d / 2 q)}\left(e^{\left(\lfloor t\rfloor_{T / M}-\lfloor s\rfloor_{T / M}\right) A}-\left[R_{T / M}\right]^{\left(\lfloor t\rfloor_{T / M}-\lfloor s\rfloor_{T / M}\right) \frac{M}{T}}\right)\right\|_{L(H)} .
\end{aligned}
$$

Lemma 6.11 hence shows that for all $\varepsilon \in(0,1+d / 2 q-d / 4-\varrho]$ it holds that

$$
\begin{aligned}
& \sup _{M \in \mathbb{N}} \sup _{t \in(0, T]} M^{\varrho} \int_{0}^{\max \left\{0,\lfloor t\rfloor_{T / M}-\frac{T}{M}\right\}}\left\|R_{\left(t-\lfloor t\rfloor_{T / M}\right)}\left(e^{\left(\lfloor t\rfloor_{T / M}-\lfloor s\rfloor_{T / M}\right) A}-\left[R_{T / M}\right]^{\left(\lfloor t\rfloor_{T / M}-\lfloor s\rfloor_{T / M}\right) \frac{M}{T}}\right)\right\|_{L\left(H, L^{q}\left(\lambda_{\mathcal{D}} ; \mathbb{R}\right)\right)} d s \\
& \leq \sup _{M \in \mathbb{N}} \sup _{l \in\{1,2, \ldots, M\}} M^{\varrho} \sum_{k=0}^{l-2} \int_{\frac{k T}{M}}^{\frac{(k+1) T}{M}} C\left\|(-A)^{(d / 4-d / 2 q)}\left(e^{\frac{(l-k) T}{M} A}-\left[R_{T / M}\right]^{(l-k)}\right)\right\|_{L(H)} d s \\
& \leq \sup _{M \in \mathbb{N}} \sup _{l \in\{1,2, \ldots, M\}}\left[M^{\varrho} \sum_{k=0}^{l-2} \frac{16 C T^{(1+d / 2 q-d / 4)}}{(l-k) M^{(1+d / 2 q-d / 4)}}\right]=\sup _{M \in\{2,3, \ldots\}} \sup _{l \in\{2,3, \ldots, M\}}\left[\sum_{k=2}^{l} \frac{16 C T^{(1+d / 2 q-d / 4)}}{k M^{(1+d / 2 q-d / 4-\varrho)}}\right] \\
& \leq \sup _{M \in \mathbb{N}}\left[\frac{16 C T^{(1+d / 2 q-d / 4)}}{M^{(1+d / 2 q-d / 4-\varrho)}} \int_{1}^{M} \frac{1}{s^{(1-\varepsilon)}} d s\right]=\sup _{M \in \mathbb{N}}\left[\frac{16 C T^{(1+d / 2 q-d / 4)}\left(M^{\varepsilon}-1\right)}{\varepsilon M^{(1+d / 2 q-d / 4-\varrho)}}\right] \\
& \leq \frac{16 C T^{(1+d / 2 q-d / 4)}}{\varepsilon}<\infty .
\end{aligned}
$$

Combining Lemma 6.9, (149), (150), (152), and (154) completes the proof of Lemma 6.12.

Lemma 6.13. Assume the setting in Section [6.1, let $p \in[1, \infty), \theta \in(0,1 / 4), \mathcal{D}=(0,1), \xi \in$ $\mathcal{L}^{p}(\mathbb{P} ; V)$, and let $\left(W_{t}\right)_{t \in[0, T]}$ be an $\operatorname{Id}_{H}$-cylindrical $\left(\mathcal{F}_{t}\right)_{t \in[0, T]}$-Wiener process. Then there exists an up to indistinguishability unique stochastic process $O:[0, T] \times \Omega \rightarrow V$ with continuous sample paths which satisfies

(i) that for all $t \in[0, T]$ it holds that $\left[O_{t}-e^{t A} \xi\right]_{\mathbb{P}, \mathcal{B}(H)}=\int_{0}^{t} e^{(t-s) A} d W_{s}$,

(ii) that for all $\omega \in \Omega, r \in[0, \theta]$ it holds that $\sup _{0 \leq s<t \leq T} \frac{s\left\|O_{t}(\omega)-O_{s}(\omega)\right\|_{V}}{(t-s)^{r}}<\infty$,

(iii) and that $\sup _{t \in[0, T]} \sup _{M \in \mathbb{N}}\|\| O_{t}\left\|_{V}+\left|M\lfloor t\rfloor_{T / M}\right|^{\theta}\right\| O_{t}-O_{\lfloor t\rfloor_{T / M}}\left\|_{V}\right\|_{\mathcal{L}^{p}(\mathbb{P} ; \mathbb{R})}<\infty$.

Proof of Lemma 6.13. Throughout this proof let $\tilde{O}:[0, T] \times \Omega \rightarrow V$ be an up to indistinguishability unique stochastic process which satisfies for all $t \in[0, T]$ that $\left[\tilde{O}_{t}\right]_{\mathbb{P}, \mathcal{B}(H)}=\int_{0}^{t} e^{(t-s) A} d W_{s}$, which 
satisfies for all $\omega \in \Omega, r \in(0, \theta]$ that $\sup _{0 \leq s<t \leq T}(t-s)^{-r}\left\|\tilde{O}_{t}(\omega)-\tilde{O}_{s}(\omega)\right\|_{V}<\infty$, and which satisfies for all $r \in(0, \theta]$ that $\sup _{0 \leq s<t \leq T}(t-s)^{-r}\left\|\tilde{O}_{t}-\tilde{O}_{s}\right\|_{\mathcal{L}^{p}(\mathbb{P} ; V)}<\infty$. Observe that, e.g., Lemma 4.3 in Blömker \& Jentzen [3] ensures that such a stochastic process does indeed exist. Next let $O:[0, T] \times \Omega \rightarrow V$ be the stochastic process with the property that for all $t \in[0, T]$ it holds that $O_{t}=e^{t A} \xi+\tilde{O}_{t}$. Note that the fact that for all $\omega \in \Omega$ it holds that the function $[0, T] \ni t \mapsto \tilde{O}_{t}(\omega) \in V$ is Hölder continuous and the fact that for all $\omega \in \Omega$ it holds that the function $[0, T] \ni t \mapsto e^{t A} \xi(\omega) \in V$ is continuous imply that for all $\omega \in \Omega$ it holds that the function $[0, T] \ni t \mapsto O_{t}(\omega) \in V$ is continuous. Moreover, observe that for all $t \in[0, T]$ it holds that $\left[O_{t}-e^{t A} \xi\right]_{\mathbb{P}, \mathcal{B}(H)}=\left[\tilde{O}_{t}\right]_{\mathbb{P}, \mathcal{B}(H)}=\int_{0}^{t} e^{(t-s) A} d W_{s}$. This implies (il). In addition, observe that Lemma 6.5, the fact that $\forall s \in[0, \infty), r \in[0,1]:\left\|(-s A)^{r} e^{s A}\right\|_{L(H)} \leq 1$, and the fact that $\forall s \in(0, \infty), r \in[0,1]:\left\|(-s A)^{-r}\left(e^{s A}-\operatorname{Id}_{H}\right)\right\|_{L(H)} \leq 1$ assure that for all $\omega \in \Omega, r \in(0, \theta]$ it holds that

$$
\begin{aligned}
& \sup _{0<s<t \leq T} \frac{s\left\|O_{t}(\omega)-O_{s}(\omega)\right\|_{V}}{(t-s)^{r}}=\sup _{0<s<t \leq T} \frac{s\left\|e^{t A} \xi(\omega)+\tilde{O}_{t}(\omega)-e^{s A} \xi(\omega)-\tilde{O}_{s}(\omega)\right\|_{V}}{(t-s)^{r}} \\
& \leq \sup _{0<s<t \leq T}\left[\frac{s\left\|e^{s A}\left(e^{(t-s) A}-\operatorname{Id}_{H}\right) \xi(\omega)\right\|_{V}}{(t-s)^{r}}+\frac{s\left\|\tilde{O}_{t}(\omega)-\tilde{O}_{s}(\omega)\right\|_{V}}{(t-s)^{r}}\right] \\
& \leq \sup _{0<s<t \leq T}\left[\frac{s\left\|e^{s / 2 A}\right\|_{L(H, V)}\left\|e^{s / 2 A}\left(e^{(t-s) A}-\operatorname{Id}_{H}\right)\right\|_{L(H)}\|\xi(\omega)\|_{H}}{(t-s)^{r}}+\frac{T\left\|\tilde{O}_{t}(\omega)-\tilde{O}_{s}(\omega)\right\|_{V}}{(t-s)^{r}}\right] \\
& \leq 2\left[\sup _{s \in(0, T]} s^{\left(1 / 4-1 / 4 n^{2}\right)}\left\|e^{s A}\right\|_{L(H, V)}\right]\left[\sup _{0<s<t \leq T}(t-s)^{\left(1-1 / 4+1 / 4 n^{2}-r\right)}\right. \\
& \left.\quad \cdot\left\|(-(t-s) A)^{-\left(1-1 / 4+1 / 4 n^{2}\right)}\left(e^{(t-s) A}-\operatorname{Id}_{H}\right)\right\|\left\|_{L(H)}\right\| \xi(\omega) \|_{H}\right]+\sup _{0<s<t \leq T} \frac{T\left\|\tilde{O}_{t}(\omega)-\tilde{O}_{s}(\omega)\right\|_{V}}{(t-s)^{r}} \\
& \leq 2 \max \{1, T\}\left[\sup _{s \in(0, T]} s^{\left(1 / 4-1 / 4 n^{2}\right)}\left\|e^{s A}\right\|_{L(H, V)}\right]\|\xi(\omega)\|_{H}+\sup _{0<s<t \leq T} \frac{T\left\|\tilde{O}_{t}(\omega)-\tilde{O}_{s}(\omega)\right\|_{V}}{(t-s)^{r}}<\infty .
\end{aligned}
$$

This shows (iii). Furthermore, note that Lemma 6.1 implies that

$$
\begin{aligned}
& \sup _{t \in[0, T]}\left\|O_{t}\right\|_{\mathcal{L}^{p}(\mathbb{P} ; V)}=\sup _{t \in[0, T]}\left\|e^{t A} \xi+\tilde{O}_{t}\right\|_{\mathcal{L}^{p}(\mathbb{P} ; V)} \\
& \leq\left[\sup _{t \in[0, T]}\left\|e^{t A}\right\|_{L(V)}\right]\|\xi\|_{\mathcal{L}^{p}(\mathbb{P} ; V)}+\sup _{t \in(0, T]}\left\|\tilde{O}_{t}\right\|_{\mathcal{L}^{p}(\mathbb{P} ; V)} \leq\|\xi\|_{\mathcal{L}^{p}(\mathbb{P} ; V)}+\sup _{t \in(0, T]} \frac{T^{\theta}\left\|\tilde{O}_{t}\right\|_{\mathcal{L}^{p}(\mathbb{P} ; V)}}{t^{\theta}}<\infty .
\end{aligned}
$$


In the next step observe that

$$
\begin{aligned}
& \sup _{M \in \mathbb{N}} \sup _{t \in[0, T]}\left[\left|M\lfloor t\rfloor_{T / M}\right|^{\theta}\left\|O_{t}-O_{\lfloor t\rfloor_{T / M}}\right\|_{\mathcal{L}^{p}(\mathbb{P} ; V)}\right] \\
& =\sup _{M \in \mathbb{N}} \sup _{t \in[0, T]}\left[\left|M\lfloor t\rfloor_{T / M}\right|^{\theta}\left\|e^{t A} \xi+\tilde{O}_{t}-e^{\lfloor t\rfloor_{T / M} A} \xi-\tilde{O}_{\lfloor t\rfloor_{T / M}}\right\|_{\mathcal{L}^{p}(\mathbb{P} ; V)}\right] \\
& \leq \sup _{M \in \mathbb{N}} \sup _{t \in[0, T] \backslash}\left[\left|M\lfloor t\rfloor_{T / M}\right|^{\theta}\left(t-\lfloor t\rfloor_{T / M}\right)^{\theta} \frac{\left\|e^{\lfloor t\rfloor_{T / M} A}\left(e^{\left(t-\lfloor t\rfloor_{T / M}\right) A}-\operatorname{Id}_{H}\right)\right\|_{L(V)}}{\left(t-\lfloor t\rfloor_{T / M}\right)^{\theta}}\|\xi\|_{\mathcal{L}^{p}(\mathbb{P} ; V)}\right] \\
& \quad+\sup _{M \in \mathbb{N}} \sup _{t \in[0, T] \backslash, T\}}\left[|M T|^{\theta}\left(t-\lfloor t\rfloor_{T / M}\right)^{\theta} \frac{\left\|\tilde{O}_{t}-\tilde{O}_{\lfloor t\rfloor_{T / M}}\right\|_{\mathcal{L}^{p}(\mathbb{P} ; V)}}{\left(t-\lfloor t\rfloor_{T / M}\right)^{\theta}}\right] .
\end{aligned}
$$

This and, e.g., Lemma 12.36 in Renardy \& Rogers [38] prove that

$$
\begin{aligned}
& \sup _{M \in \mathbb{N}} \sup _{t \in[0, T]}\left[\left|M\lfloor t\rfloor_{T / M}\right|^{\theta}\left\|O_{t}-O_{\lfloor t\rfloor_{T / M}}\right\|_{\mathcal{L}^{p}(\mathbb{P} ; V)}\right] \\
& \leq T^{\theta}\left[\sup _{M \in \mathbb{N}} \sup _{\substack{t \in[0, T\rfloor \backslash \\
\left\{0, \frac{T}{M}, \ldots, T\right\}}}\left\|\left(-\lfloor t\rfloor_{T / M} A\right)^{\theta} e^{\lfloor t\rfloor_{T / M} A}\right\|_{L(V)}\right. \\
& \left.\cdot\left\|\left(-\left(t-\lfloor t\rfloor_{T / M}\right) A\right)^{-\theta}\left(e^{\left(t-\lfloor t\rfloor_{T / M}\right) A}-\operatorname{Id}_{H}\right)\right\|_{L(V)}\right]\|\xi\|_{\mathcal{L}^{p}(\mathbb{P} ; V)}+\sup _{0 \leq s<t \leq T}\left[\frac{T^{2 \theta}\left\|\tilde{O}_{t}-\tilde{O}_{s}\right\|_{\mathcal{L}^{p}(\mathbb{P} ; V)}}{(t-s)^{\theta}}\right] \\
& \leq T^{\theta}\left[\sup _{s \in(0, T)}\left\|(-s A)^{\theta} e^{s A}\right\|_{L(V)}\right]\left[\sup _{s \in(0, T)}\left\|(-s A)^{-\theta}\left(e^{s A}-\operatorname{Id}_{H}\right)\right\|_{L(V)}\right]\|\xi\|_{\mathcal{L}^{p}(\mathbb{P} ; V)} \\
& \quad+\sup _{0 \leq s<t \leq T}\left[\frac{T^{2 \theta}\left\|\tilde{O}_{t}-\tilde{O}_{s}\right\|_{\mathcal{L}^{p}(\mathbb{P} ; V)}}{(t-s)^{\theta}}\right]<\infty .
\end{aligned}
$$

Combining (156) and (158) shows (iii). The proof of Lemma 6.13 is thus completed.

\subsection{Strong convergence rates for a nonlinearity-truncated exponential Euler scheme}

Corollary 6.14. Assume the setting in Section [6.1, let $\vartheta \in(0, \infty), \chi \in\left(0, \frac{1}{2 n}\right], p \in[2, \infty)$, $\mathcal{D}=(0,1), \xi \in \mathcal{L}^{(3 n-1) p \max \{2,2(n-1), 2 \vartheta, 2(n-1) \vartheta\}}(\mathbb{P} ; V)$, let $\left(W_{t}\right)_{t \in[0, T]}$ be an $\operatorname{Id}_{H^{-}}$cylindrical $\left(\mathcal{F}_{t}\right)_{t \in[0, T]^{-}}$ Wiener process, let $X:[0, T] \times \Omega \rightarrow V$ be a stochastic process with continuous sample paths which satisfies for all $t \in[0, T]$ that $\left[X_{t}-e^{t A} \xi-\int_{0}^{t} e^{(t-s) A} F\left(X_{s}\right) d s\right]_{\mathbb{P}, \mathcal{B}(H)}=\int_{0}^{t} e^{(t-s) A} d W_{s}$, and let $\mathcal{X}^{M}:[0, T] \times \Omega \rightarrow V, M \in \mathbb{N}$, be stochastic processes which satisfy for all $t \in[0, T], M \in \mathbb{N}$ that

$$
\left[\mathcal{X}_{t}^{M}-e^{t A} \xi-\int_{0}^{t} e^{\left(t-\lfloor s\rfloor_{T / M}\right) A} \mathbb{1}_{\left\{\left\|\mathcal{X}_{\left.\mathcal{L}^{M}\right\rfloor_{T / M}}\right\|_{V} \leq(M / T)^{\chi}\right\}} F\left(\mathcal{X}_{\lfloor s\rfloor_{T / M}}^{M}\right) d s\right]_{\mathbb{P}, \mathcal{B}(H)}=\int_{0}^{t} e^{\left(t-\lfloor s\rfloor_{T / M}\right) A} d W_{s}
$$


Then it holds for all $\theta \in[0,1 / 4)$ that

$$
\sup _{M \in \mathbb{N}} \sup _{t \in[0, T]}\left[\left\|\mathcal{X}_{t}^{M}\right\|_{\mathcal{L}^{p \max \{2,2(n-1), 4 \vartheta, 4(n-1) \vartheta\}(\mathbb{P} ; V)}}+\left\|X_{t}\right\|_{\mathcal{L}^{p}(\mathbb{P} ; H)}+M^{\min \{\vartheta \chi, \theta\}}\left\|X_{t}-\mathcal{X}_{t}^{M}\right\|_{\mathcal{L}^{p}(\mathbb{P} ; H)}\right]<\infty .
$$

Proof of Corollary 6.14. Throughout this proof let $\mathcal{O}^{M}:[0, T] \times \Omega \rightarrow V, M \in \mathbb{N}$, be stochastic processes with the property that for all $t \in[0, T], M \in \mathbb{N}$ it holds that $\left[\mathcal{O}_{t}^{M}-e^{t A} \xi\right]_{\mathbb{P}, \mathcal{B}(H)}=$ $\int_{0}^{t} e^{\left(t-\lfloor s\rfloor_{T / M}\right) A} d W_{s}$. Next note that Lemma 6.13 ensures that there exists a stochastic process $O:[0, T] \times \Omega \rightarrow V$ with continuous sample paths which satisfies for all $t \in[0, T]$ that $\left[O_{t}-\right.$ $\left.e^{t A} \xi\right]_{\mathbb{P}, \mathcal{B}(H)}=\int_{0}^{t} e^{(t-s) A} d W_{s}$, which satisfies for all $\omega \in \Omega$ that $\lim \sup _{r \searrow 0} \sup _{0 \leq s<t \leq T} \frac{s\left\|O_{t}(\omega)-O_{s}(\omega)\right\|_{V}}{(t-s)^{r}}<$ $\infty$, and which satisfies for all $\theta \in(0,1 / 4)$ that

$$
\sup _{M \in \mathbb{N}} \sup _{t \in[0, T]}\|\| O_{t}\left\|_{V}+\left|M\lfloor t\rfloor_{T / M}\right|^{\theta}\right\| O_{t}-O_{\lfloor t\rfloor_{T / M}}\left\|_{V}\right\|_{\mathcal{L}^{(3 n-1) p \max \{2,2(n-1), 2 \vartheta, 2(n-1) \vartheta\}(\mathbb{P} ; \mathbb{R})}}<\infty .
$$

Furthermore, note that for all $q \in[1, \infty), \theta \in[0,1 / 4)$ it holds that

$$
\begin{aligned}
& \sup _{M \in \mathbb{N}} \sup _{t \in[0, T]} M^{\theta}\left\|O_{t}-\mathcal{O}_{t}^{M}\right\|_{\mathcal{L}^{q}(\mathbb{P} ; V)} \\
& \leq \sup _{M \in \mathbb{N}} M^{\theta}\left(\mathbb{E}\left[\sup _{t \in[0, T]}\left\|O_{t}-\mathcal{O}_{t}^{M}\right\|_{V}^{q}\right]\right)^{1 / q} \leq \sup _{M \in \mathbb{N}} M^{\theta}\left(\mathbb{E}\left[\sup _{t \in[0, T]}\left\|O_{t}-\mathcal{O}_{t}^{M}\right\|_{\mathcal{C}([0,1], \mathbb{R})}^{q}\right]\right)^{1 / q}<\infty
\end{aligned}
$$

(cf., e.g., Theorem 4.13 in Cox \& van Neerven [5]). This, the triangle inequality, and (161) show that

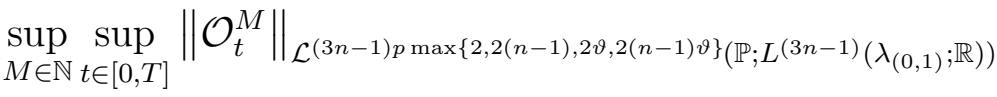

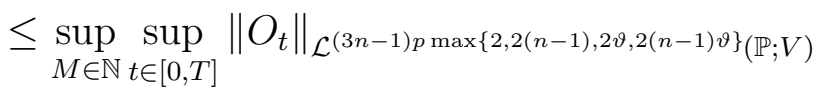

$$
\begin{aligned}
& +\sup _{M \in \mathbb{N}} \sup _{t \in[0, T]}\left\|O_{t}-\mathcal{O}_{t}^{M}\right\|_{\mathcal{L}^{(3 n-1) p \max \{2,2(n-1), 2 \vartheta, 2(n-1) \vartheta\}(\mathbb{P} ; V)}}<\infty .
\end{aligned}
$$

Moreover, note that Corollary 6.3 ensures that there exists a real number $K \in(0, \infty)$ which satisfies for all $h \in(0, T], t \in[0, h], u, v \in V$ that

$$
\begin{aligned}
& \left\|e^{t A}\left[u+t \mathbb{1}_{\left[0, h^{-\chi}\right]}\left(\|u+v\|_{V}\right) F(u+v)\right]\right\|_{L^{2 n}\left(\lambda_{(0,1)} ; \mathbb{R}\right)}^{2 n} \\
& \leq e^{t}\left[\|u\|_{L^{2 n}\left(\lambda_{(0,1)} ; \mathbb{R}\right)}^{2 n}+t K \max \left\{1,\|v\|_{L^{(3 n-1)}\left(\lambda_{(0,1)} ; \mathbb{R}\right)}^{(3 n-1)}\right\}\right] .
\end{aligned}
$$

In the next step observe that inequalities (161)-(163) imply that for all $\theta \in(0,1 / 4)$ it holds that

$$
\begin{aligned}
& \sup _{M \in \mathbb{N}} \sup _{t \in[0, T]}\|\| \mathcal{O}_{t}^{M}\left\|_{L^{2 n}\left(\lambda_{(0,1)} ; \mathbb{R}\right)}^{2 n}+K \max \left\{1,\left\|\mathcal{O}_{t}^{M}\right\|_{L^{(3 n-1)}\left(\lambda_{(0,1)} ; \mathbb{R}\right)}^{(3 n-1)}\right\}\right\|_{\mathcal{L}^{p \max \{2,2(n-1), 2 \vartheta, 2(n-1) \vartheta\}(\mathbb{P} ; \mathbb{R})}}
\end{aligned}
$$

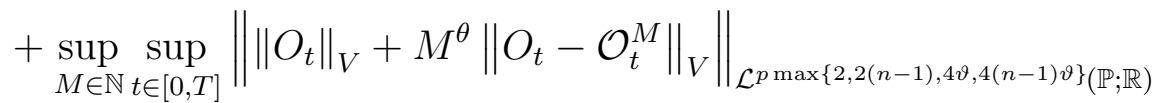

$$
\begin{aligned}
& +\sup _{M \in \mathbb{N}} \sup _{t \in[0, T]}\left[\left|M\lfloor t\rfloor_{T / M}\right|^{\theta}\left\|O_{t}-O_{\lfloor t\rfloor_{T / M}}\right\|_{\mathcal{L}^{2 p}(\mathbb{P} ; V)}\right]<\infty \text {. }
\end{aligned}
$$


Combining this and the fact that $\forall t_{1}, t_{2}, t_{3} \in[0, T]$ with $t_{1}<t_{2}<t_{3}: e^{\left(t_{3}-t_{1}\right) A}=e^{\left(t_{3}-t_{2}\right) A} e^{\left(t_{2}-t_{1}\right) A}$ with (164), Lemma 6.5, Lemma 6.6. Lemma 6.7, Lemma 6.8, and Lemma 6.9 allows us to apply Theorem 5.3 (with $\varphi=\max \{1,2(n-1)\}, \phi=1 / 2, \mathcal{S}_{s, t}^{M}=e^{(t-s) A}, \Phi(v)=\|v\|_{L^{2 n}\left(\lambda_{(0,1)} ; \mathbb{R}\right)}^{2 n}, \Psi(v)=$ $K \max \left\{1,\|v\|_{L^{(3 n-1)}\left(\lambda_{(0,1)} ; \mathbb{R}\right)}^{(3 n-1)}\right\}, \rho=1 / 4-1 / 4 n^{2}$, and $\varrho=3 / 4$ for $v \in V, M \in \mathbb{N}, s, t \in[0, T]$ with $s<t$ in the notation of Theorem [5.3) to obtain (160). The proof of Corollary 6.14 is thus completed.

The following result, Corollary 6.15, specializes Corollary 6.14 to the case where the initial random variable $\xi: \Omega \rightarrow V$ satisfies $\forall p \in(0, \infty): \mathbb{E}\left[\|\xi\|_{V}^{p}\right]<\infty$.

Corollary 6.15. Assume the setting in Section [6.1, let $\mathcal{D}=(0,1), \xi \in \cap_{p \in(0, \infty)} \mathcal{L}^{p}(\mathbb{P} ; V), \chi \in$ $\left(0, \frac{1}{2 n}\right]$, let $\left(W_{t}\right)_{t \in[0, T]}$ be an $\operatorname{Id}_{H}$-cylindrical $\left(\mathcal{F}_{t}\right)_{t \in[0, T]}$-Wiener process, let $X:[0, T] \times \Omega \rightarrow V$ be a stochastic process with continuous sample paths which satisfies for all $t \in[0, T]$ that $\left[X_{t}-e^{t A} \xi-\right.$ $\left.\int_{0}^{t} e^{(t-s) A} F\left(X_{s}\right) d s\right]_{\mathbb{P}, \mathcal{B}(H)}=\int_{0}^{t} e^{(t-s) A} d W_{s}$, and let $\mathcal{X}^{M}:[0, T] \times \Omega \rightarrow V, M \in \mathbb{N}$, be stochastic processes which satisfy for all $t \in[0, T], M \in \mathbb{N}$ that

$$
\left[\mathcal{X}_{t}^{M}-e^{t A} \xi-\int_{0}^{t} e^{\left(t-\lfloor s\rfloor_{T / M}\right) A} \mathbb{1}_{\left\{\left\|\mathcal{X}_{\mathcal{L}\rfloor_{T / M}^{M}}\right\|_{V} \leq(M / T)^{\chi}\right\}} F\left(\mathcal{X}_{\lfloor s\rfloor_{T / M}}^{M}\right) d s\right]_{\mathbb{P}, \mathcal{B}(H)}=\int_{0}^{t} e^{\left(t-\lfloor s\rfloor_{T / M}\right) A} d W_{s}
$$

Then it holds for all $\theta \in[0,1 / 4), p \in(0, \infty)$ that

$$
\sup _{M \in \mathbb{N}} \sup _{t \in[0, T]}\left[\left\|\mathcal{X}_{t}^{M}\right\|_{\mathcal{L}^{p}(\mathbb{P} ; V)}+\left\|X_{t}\right\|_{\mathcal{L}^{p}(\mathbb{P} ; H)}+M^{\theta}\left\|X_{t}-\mathcal{X}_{t}^{M}\right\|_{\mathcal{L}^{p}(\mathbb{P} ; H)}\right]<\infty
$$

\subsection{Strong convergence rates for a nonlinearity-truncated linear-implicit Euler scheme}

Corollary 6.16. Assume the setting in Section [6.1, let $\vartheta \in(0, \infty), \chi \in\left(0, \frac{1}{2 n}\right], p \in[2, \infty)$, $\mathcal{D}=(0,1), \xi \in \mathcal{L}^{(3 n-1) p \max \{2,2(n-1), 2 \vartheta, 2(n-1) \vartheta\}}\left(\mathbb{P} ; H_{3 / 4}\right)$, let $\left(W_{t}\right)_{t \in[0, T]}$ be an $\operatorname{Id}_{H^{-}}$cylindrical $\left(\mathcal{F}_{t}\right)_{t \in[0, T]^{-}}$ Wiener process, let $X:[0, T] \times \Omega \rightarrow V$ be a stochastic process with continuous sample paths which satisfies for all $t \in[0, T]$ that $\left[X_{t}-e^{t A} \xi-\int_{0}^{t} e^{(t-s) A} F\left(X_{s}\right) d s\right]_{\mathbb{P}, \mathcal{B}(H)}=\int_{0}^{t} e^{(t-s) A} d W_{s}$, and let $\mathcal{X}^{M}:[0, T] \times \Omega \rightarrow V, M \in \mathbb{N}$, be stochastic processes which satisfy for all $t \in[0, T], M \in \mathbb{N}$ that

$$
\begin{aligned}
& {\left[\mathcal{X}_{t}^{M}-\left(\operatorname{Id}_{H}-\left(t-\lfloor t\rfloor_{T / M}\right) A\right)^{-1}\left(\operatorname{Id}_{H}-\frac{T}{M} A\right)^{-\lfloor t\rfloor_{T / M} \frac{M}{T}} \xi\right.} \\
& \left.-\int_{0}^{t}\left(\operatorname{Id}_{H}-\left(t-\lfloor t\rfloor_{T / M}\right) A\right)^{-1}\left(\operatorname{Id}_{H}-\frac{T}{M} A\right)^{\left(\lfloor s\rfloor_{T / M}-\lfloor t\rfloor_{T / M}\right) \frac{M}{T}} \mathbb{1}_{\left\{\left\|\mathcal{X}_{\lfloor s\rfloor_{T / M}^{M}}^{M}\right\|_{V} \leq(M / T)^{\chi}\right\}} F\left(\mathcal{X}_{\lfloor s\rfloor_{T / M}}^{M}\right) d s\right]_{\mathbb{P}, \mathcal{B}(H)} \\
& =\int_{0}^{t}\left(\operatorname{Id}_{H}-\left(t-\lfloor t\rfloor_{T / M}\right) A\right)^{-1}\left(\operatorname{Id}_{H}-\frac{T}{M} A\right)^{\left(\lfloor s\rfloor_{T / M}-\lfloor t\rfloor_{T / M}\right) \frac{M}{T}} d W_{s} .
\end{aligned}
$$

Then it holds for all $\theta \in[0,1 / 4)$ that

$$
\sup _{M \in \mathbb{N}} \sup _{t \in[0, T]}\left[\left\|\mathcal{X}_{t}^{M}\right\|_{\mathcal{L}^{p \max \{2,2(n-1), 4 \vartheta, 4(n-1) \vartheta\}(\mathbb{P} ; V)}}+\left\|X_{t}\right\|_{\mathcal{L}^{p}(\mathbb{P} ; H)}+M^{\min \{\vartheta \chi, \theta\}}\left\|X_{t}-\mathcal{X}_{t}^{M}\right\|_{\mathcal{L}^{p}(\mathbb{P} ; H)}\right]<\infty .
$$


Proof of Corollary 6.16. Throughout this proof let $\mathcal{O}^{M}:[0, T] \times \Omega \rightarrow V, M \in \mathbb{N}$, be stochastic processes with the property that for all $t \in[0, T], M \in \mathbb{N}$ it holds that $\left[\mathcal{O}_{t}^{M}-\left(\operatorname{Id}_{H}-(t-\right.\right.$ $\left.\left.\left.\lfloor t\rfloor_{T / M}\right) A\right)^{-1}\left(\operatorname{Id}_{H}-\frac{T}{M} A\right)^{-\lfloor t\rfloor_{T / M} \frac{M}{T} \xi}\right]_{\mathbb{P}, \mathcal{B}(H)}=\int_{0}^{t}\left(\operatorname{Id}_{H}-\left(t-\lfloor t\rfloor_{T / M}\right) A\right)^{-1}\left(\operatorname{Id}_{H}-\frac{T}{M} A\right)^{\left(\lfloor s\rfloor_{T / M}-\lfloor t\rfloor_{T / M}\right) \frac{M}{T}} d W_{s}$. Next note that Lemma 6.13 together with the fact that $H_{3 / 4} \subseteq V$ continuously ensures that there exists a stochastic process $O:[0, T] \times \Omega \rightarrow V$ with continuous sample paths which satisfies for all $\omega \in \Omega$ that $\lim \sup _{r}{ }^{0} \sup _{0 \leq s<t \leq T} \frac{s\left\|O_{t}(\omega)-O_{s}(\omega)\right\|_{V}}{(t-s)^{r}}<\infty$, which satisfies for all $t \in[0, T]$ that $\left[O_{t}-e^{t A} \xi\right]_{\mathbb{P}, \mathcal{B}(H)}=\int_{0}^{t} e^{(t-s) A} d W_{s}$, and which satisfies for all $\theta \in(0,1 / 4)$ that

$$
\sup _{M \in \mathbb{N}} \sup _{t \in[0, T]}\|\| O_{t}\left\|_{V}+\left|M\lfloor t\rfloor_{T / M}\right|^{\theta}\right\| O_{t}-O_{\lfloor t\rfloor_{T / M}}\left\|_{V}\right\|_{\mathcal{L}^{(3 n-1) p \max \{2,2(n-1), 2 \vartheta, 2(n-1) \vartheta\}(\mathbb{P} ; \mathbb{R})}}<\infty .
$$

Moreover, note that for all $q \in[1, \infty), \theta \in[0,1 / 4)$ it holds that

$$
\sup _{M \in \mathbb{N}} \sup _{t \in[0, T]} M^{\theta}\left\|O_{t}-\mathcal{O}_{t}^{M}\right\|_{\mathcal{L}^{q}(\mathbb{P} ; V)} \leq \sup _{M \in \mathbb{N}} M^{\theta}\left(\mathbb{E}\left[\sup _{t \in[0, T]}\left\|O_{t}-\mathcal{O}_{t}^{M}\right\|_{V}^{q}\right]\right)^{1 / q}<\infty
$$

(cf., e.g., Theorem 1.1 in Cox \& van Neerven [6]). The triangle inequality and (170) therefore show that

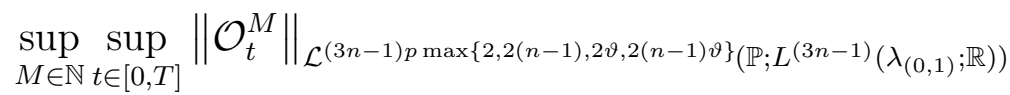

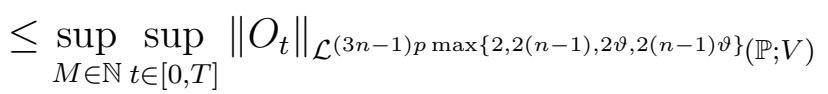

$$
\begin{aligned}
& +\sup _{M \in \mathbb{N}} \sup _{t \in[0, T]}\left\|O_{t}-\mathcal{O}_{t}^{M}\right\|_{\mathcal{L}^{(3 n-1) p \max \{2,2(n-1), 2 \vartheta, 2(n-1) \vartheta\}(\mathbb{P} ; V)}}<\infty .
\end{aligned}
$$

Next note that Corollary 6.3 ensures that there exists a real number $K \in(0, \infty)$ which satisfies for all $h \in(0, T], t \in[0, h], u, v \in V$ that

$$
\begin{aligned}
& \left\|\left(\operatorname{Id}_{H}-t A\right)^{-1}\left[u+t \mathbb{1}_{\left[0, h^{-\chi}\right]}\left(\|u+v\|_{V}\right) F(u+v)\right]\right\|_{L^{2 n}\left(\lambda_{(0,1)} ; \mathbb{R}\right)}^{2 n} \\
& \leq e^{t}\left[\|u\|_{L^{2 n}\left(\lambda_{(0,1)} ; \mathbb{R}\right)}^{2 n}+t K \max \left\{1,\|v\|_{L^{(3 n-1)}\left(\lambda_{(0,1)} ; \mathbb{R}\right)}^{(3 n-1)}\right\}\right] .
\end{aligned}
$$

Moreover, inequalities (170)-(172) assure that for all $\theta \in(0,1 / 4)$ it holds that

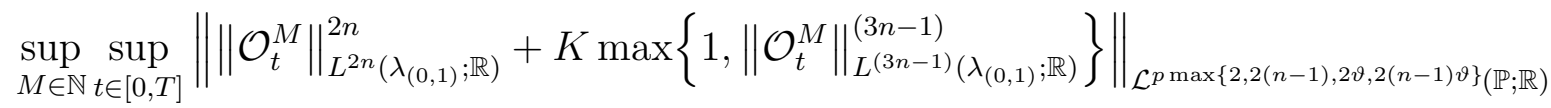

$$
\begin{aligned}
& +\sup _{M \in \mathbb{N}} \sup _{t \in[0, T]}\|\| O_{t}\left\|_{V}+M^{\theta}\right\| O_{t}-\mathcal{O}_{t}^{M}\left\|_{V}\right\|_{\mathcal{L}^{p \max \{2,2(n-1), 4 \vartheta, 4(n-1) \vartheta\}(\mathbb{P} ; \mathbb{R})}} \\
& +\sup _{M \in \mathbb{N}} \sup _{t \in[0, T]}\left[\left|M\lfloor t\rfloor_{T / M}\right|^{\theta}\left\|O_{t}-O_{\lfloor t\rfloor_{T / M}}\right\|_{\mathcal{L}^{2 p}(\mathbb{P} ; V)}\right]<\infty .
\end{aligned}
$$

In addition, observe that for all $t_{1}, t_{2}, t_{3} \in[0, T]$ with $t_{1}<t_{2}<t_{3}$ it holds that

$$
\begin{aligned}
& \left(\operatorname{Id}_{H}-\left(t_{1}-\left\lfloor t_{1}\right\rfloor_{T / M}\right) A\right)\left(\operatorname{Id}_{H}-\left(t_{3}-\left\lfloor t_{3}\right\rfloor_{T / M}\right) A\right)^{-1}\left(\operatorname{Id}_{H}-\frac{T}{M} A\right)^{\left(\left\lfloor t_{1}\right\rfloor_{T / M}-\left\lfloor t_{3}\right\rfloor_{T / M}\right) \frac{T}{M}} \\
& =\left(\operatorname{Id}_{H}-\left(t_{2}-\left\lfloor t_{2}\right\rfloor_{T / M}\right) A\right)\left(\operatorname{Id}_{H}-\left(t_{3}-\left\lfloor t_{3}\right\rfloor_{T / M}\right) A\right)^{-1}\left(\operatorname{Id}_{H}-\frac{T}{M} A\right)^{\left(\left\lfloor t_{2}\right\rfloor_{T / M}-\left\lfloor t_{3}\right\rfloor_{T / M}\right) \frac{T}{M}} \\
& \quad\left(\operatorname{Id}_{H}-\left(t_{1}-\left\lfloor t_{1}\right\rfloor_{T / M}\right) A\right)\left(\operatorname{Id}_{H}-\left(t_{2}-\left\lfloor t_{2}\right\rfloor_{T / M}\right) A\right)^{-1}\left(\operatorname{Id}_{H}-\frac{T}{M} A\right)^{\left(\left\lfloor t_{1}\right\rfloor_{T / M}-\left\lfloor t_{2}\right\rfloor_{T / M}\right) \frac{T}{M}}
\end{aligned}
$$


(cf., e.g., (142)-(146) in Da Prato et al. [7]). Combining this, (173)-(174), Lemma 6.5, Lemma 6.6, Lemma 6.7, Lemma 6.8, and Lemma 6.12 ensures that we can apply Theorem 5.3 (with $\varphi=$ $\max \{1,2(n-1)\}, \phi=1 / 2, \Phi(v)=\|v\|_{L^{2 n}\left(\lambda_{(0,1)} ; \mathbb{R}\right)}^{2 n}, \Psi(v)=K \max \left\{1,\|v\|_{L^{(3 n-1)\left(\lambda_{(0,1)} ; \mathbb{R}\right)}}^{(3 n-1)}\right\}, \mathcal{S}_{s, t}^{M}=$ $\left(\operatorname{Id}_{H}-\left(s-\lfloor s\rfloor_{T / M}\right) A\right)\left(\operatorname{Id}_{H}-\left(t-\lfloor t\rfloor_{T / M}\right) A\right)^{-1}\left(\operatorname{Id}_{H}-\frac{T}{M} A\right)^{\left(\lfloor s\rfloor_{T / M}-\lfloor t\rfloor_{T / M}\right) \frac{T}{M}}, \rho=1 / 4-1 / 4 n^{2}$, and $\varrho=3 / 4$ for $v \in V, M \in \mathbb{N}, s, t \in[0, T]$ with $s<t$ in the notation of Theorem [5.3) to obtain (169). The proof of Corollary 6.16 is thus completed.

In our next result, Corollary 6.17, we specialize Corollary 6.16 to the case where the initial random variable $\xi: \Omega \rightarrow H_{3 / 4}$ satisfies $\forall p \in(0, \infty): \mathbb{E}\left[\|\xi\|_{H_{3 / 4}}^{p}\right]<\infty$.

Corollary 6.17. Assume the setting in Section [6.1, let $\mathcal{D}=(0,1), \xi \in \cap_{p \in(0, \infty)} \mathcal{L}^{p}\left(\mathbb{P} ; H_{3 / 4}\right), \chi \in$ $\left(0, \frac{1}{2 n}\right]$, let $\left(W_{t}\right)_{t \in[0, T]}$ be an $\operatorname{Id}_{H}$-cylindrical $\left(\mathcal{F}_{t}\right)_{t \in[0, T]}$-Wiener process, let $X:[0, T] \times \Omega \rightarrow V$ be a stochastic process with continuous sample paths which satisfies for all $t \in[0, T]$ that $\left[X_{t}-e^{t A} \xi-\right.$ $\left.\int_{0}^{t} e^{(t-s) A} F\left(X_{s}\right) d s\right]_{\mathbb{P}, \mathcal{B}(H)}=\int_{0}^{t} e^{(t-s) A} d W_{s}$, and let $\mathcal{X}^{M}:[0, T] \times \Omega \rightarrow V, M \in \mathbb{N}$, be stochastic processes which satisfy for all $t \in[0, T], M \in \mathbb{N}$ that

$$
\begin{aligned}
& {\left[\mathcal{X}_{t}^{M}-\left(\operatorname{Id}_{H}-\left(t-\lfloor t\rfloor_{T / M}\right) A\right)^{-1}\left(\operatorname{Id}_{H}-\frac{T}{M} A\right)^{-\lfloor t\rfloor_{T / M} \frac{M}{T}} \xi\right.} \\
& \left.-\int_{0}^{t}\left(\operatorname{Id}_{H}-\left(t-\lfloor t\rfloor_{T / M}\right) A\right)^{-1}\left(\operatorname{Id}_{H}-\frac{T}{M} A\right)^{\left(\lfloor s\rfloor_{T / M}-\lfloor t\rfloor_{T / M}\right) \frac{M}{T}} \mathbb{1}_{\left\{\left\|\mathcal{X}_{\lfloor s\rfloor_{T / M}^{M}}\right\|_{V} \leq(M / T) \chi\right\}} F\left(\mathcal{X}_{\lfloor s\rfloor_{T / M}}^{M}\right) d s\right]_{\mathbb{P}, \mathcal{B}(H)} \\
& =\int_{0}^{t}\left(\operatorname{Id}_{H}-\left(t-\lfloor t\rfloor_{T / M}\right) A\right)^{-1}\left(\operatorname{Id}_{H}-\frac{T}{M} A\right)^{\left(\lfloor s\rfloor_{T / M}-\lfloor t\rfloor_{T / M}\right) \frac{M}{T}} d W_{s} .
\end{aligned}
$$

Then it holds for all $\theta \in[0,1 / 4), p \in(0, \infty)$ that

$$
\sup _{M \in \mathbb{N}} \sup _{t \in[0, T]}\left[\left\|\mathcal{X}_{t}^{M}\right\|_{\mathcal{L}^{p}(\mathbb{P} ; V)}+\left\|X_{t}\right\|_{\mathcal{L}^{p}(\mathbb{P} ; H)}+M^{\theta}\left\|X_{t}-\mathcal{X}_{t}^{M}\right\|_{\mathcal{L}^{p}(\mathbb{P} ; H)}\right]<\infty .
$$

\section{Acknowledgments}

This work has been partially supported through the SNSF-Research project 200021_156603 "Numerical approximations of nonlinear stochastic ordinary and partial differential equations".

\section{References}

[1] Beyn, W.-J., IsaAk, E., And Kruse, R. Stochastic C-stability and B-consistency of explicit and implicit Euler-type schemes. J. Sci. Comput. (2015), 1-33.

[2] Beyn, W.-J., IsaAk, E., And Kruse, R. Stochastic C-stability and B-consistency of explicit and implicit Milstein-type schemes. arXiv:1512.06905 (2015), 35 pages.

[3] Blömker, D., And Jentzen, A. Galerkin approximations for the stochastic Burgers equation. SIAM J. Numer. Anal. 51, 1 (2013), 694-715. 
[4] Cerrai, S. Second order PDE's in finite and infinite dimension, vol. 1762 of Lecture Notes in Mathematics. Springer-Verlag, Berlin, 2001. A probabilistic approach.

[5] Cox, S., And van Neerven, J. Convergence rates of the splitting scheme for parabolic linear stochastic Cauchy problems. SIAM J. Numer. Anal. 48, 2 (2010), 428-451.

[6] Cox, S., And van Neerven, J. Pathwise Hölder convergence of the implicit-linear Euler scheme for semi-linear SPDEs with multiplicative noise. Numer. Math. 125, 2 (2013), 259-345.

[7] Da Prato, G., Jentzen, A., And Roeckner, M. A mild Ito formula for SPDEs. arXiv:1009.3526 (2012), 39 pages.

[8] Da Prato, G., And ZabczyK, J. Stochastic equations in infinite dimensions, vol. 44 of Encyclopedia of Mathematics and its Applications. Cambridge University Press, Cambridge, 1992.

[9] Da Prato, G., And ZabczyK, J. Ergodicity for infinite-dimensional systems, vol. 229 of London Mathematical Society Lecture Note Series. Cambridge University Press, Cambridge, 1996.

[10] Dareiotis, K., Kumar, C., And Sabanis, S. On tamed Euler approximations of SDEs driven by Lévy noise with applications to delay equations. arXiv:1403.0498 (2015), 27 pages.

[11] Gyöngy, I., And Millet, A. On discretization schemes for stochastic evolution equations. Potential Anal. 23, 2 (2005), 99-134.

[12] Gyöngy, I., SABAnis, S., And ŠišKA, D. Convergence of tamed Euler schemes for a class of stochastic evolution equations. arXiv:1308.1796 (2015), 17 pages.

[13] Halidias, N. A novel approach to construct numerical methods for stochastic differential equations. Numer. Algorithms 66, 1 (2013), 79-87.

[14] Halidias, N. Construction of positivity preserving numerical schemes for some multidimensional stochastic differential equations. Discrete Contin. Dyn. Syst. Ser. B 20, 1 (2015), 153160.

[15] Halidias, N., And Stamatiou, I. S. On the numerical solution of some nonlinear stochastic differential equations using the semi-discrete method. arXiv:1309.3189 (2014), 36 pages.

[16] Hu, Y. Semi-implicit Euler-Maruyama scheme for stiff stochastic equations. In Stochastic analysis and related topics, V (Silivri, 1994), vol. 38 of Progr. Probab. Birkhäuser Boston, Boston, MA, 1996, pp. 183-202.

[17] Hutzenthaler, M., And Jentzen, A. On a perturbation theory and on strong convergence rates for stochastic ordinary and partial differential equations with non-globally monotone coefficients. arXiv:1401.0295 (2014), 41 pages.

[18] Hutzenthaler, M., And Jentzen, A. Numerical approximations of stochastic differential equations with non-globally Lipschitz continuous coefficients. Mem. Amer. Math. Soc. 236, 1112 (2015), v+99. 
[19] Hutzenthaler, M., Jentzen, A., And Kloeden, P. E. Strong and weak divergence in finite time of Euler's method for stochastic differential equations with non-globally Lipschitz continuous coefficients. Proc. R. Soc. Lond. Ser. A Math. Phys. Eng. Sci. 467 (2011), 15631576.

[20] Hutzenthaler, M., Jentzen, A., And Kloeden, P. E. Strong convergence of an explicit numerical method for SDEs with non-globally Lipschitz continuous coefficients. Ann. Appl. Probab. 22, 4 (2012), 1611-1641.

[21] Hutzenthaler, M., Jentzen, A., And Kloeden, P. E. Divergence of the multilevel Monte Carlo Euler method for nonlinear stochastic differential equations. Ann. Appl. Probab. 23, 5 (2013), 1913-1966.

[22] Hutzenthaler, M., Jentzen, A., And Wang, X. Exponential integrability properties of numerical approximation processes for nonlinear stochastic differential equations. arXiv:1309.7657 (2014), 32 pages.

[23] Jentzen, A. Numerical analysis of stochastic partial differential equations. Lecture notes, ETH Zurich, summer semester 2015, available online at http://www.math.ethz.ch/education/bachelor/lectures/fs2015/math/numsol, May 2015.

[24] Jentzen, A., And Kurniawan, R. Weak convergence rates for Euler-type approximations of semilinear stochastic evolution equations with nonlinear diffusion coefficients. arXiv:1501.03539 (2015), 51 pages.

[25] Jentzen, A., And Pušnik, P. Strong convergence rates for an explicit numerical approximation method for stochastic evolution equations with non-globally Lipschitz continuous nonlinearities. arXiv:1504.03523 (2015), 38 pages.

[26] Kovács, M., Larsson, S., and Lindgren, F. On the discretization in time of the stochastic Allen-Cahn equation. arXiv:1510.03684 (2015), 34 pages.

[27] Kumar, C., and Sabanis, S. On tamed Milstein schemes of SDEs driven by Lévy noise. arXiv:1407.5347 (2015), 45 pages.

[28] Kumar, C., And Sabanis, S. On Milstein approximations with varying coefficients: the case of super-linear diffusion coefficients. arXiv:1601.02695 (2016), 31 pages.

[29] Kurniawan, R. Numerical approximations of stochastic partial differential equations with non-globally Lipschitz continuous nonlinearities. University of Zurich and ETH Zurich, Zurich, Switzerland, 2014. 74 pages. Master thesis.

[30] Liu, W., And MaO, X. Strong convergence of the stopped Euler-Maruyama method for nonlinear stochastic differential equations. Appl. Math. Comput. 223 (2013), $389-400$.

[31] Lord, G. J., And Rougemont, J. A numerical scheme for stochastic PDEs with Gevrey regularity. IMA J. Numer. Anal. 24, 4 (2004), 587-604. 
[32] Lord, G. J., And TAmbue, A. Stochastic exponential integrators for the finite element discretization of SPDEs for multiplicative and additive noise. IMA J. Numer. Anal. 33, 2 (2013), 515-543.

[33] Lunardi, A. Interpolation theory, second ed. Appunti. Scuola Normale Superiore di Pisa (Nuova Serie). [Lecture Notes. Scuola Normale Superiore di Pisa (New Series)]. Edizioni della Normale, Pisa, 2009.

[34] Mao, X. The truncated Euler-Maruyama method for stochastic differential equations. $J$. Comput. Appl. Math. 290 (2015), $370-384$.

[35] MaO, X. Convergence rates of the truncated Euler-Maruyama method for stochastic differential equations. J. Comput. Appl. Math. 296 (2016), $362-375$.

[36] NGO, H.-L., And Luong, D.-T. Strong rate of tamed Euler-Maruyama approximation for stochastic differential equations with Hölder continuous diffusion coefficient. To appear in Braz. J. Prob. Stat., available online at http://imstat.org/bjps/papers/BJPS301.pdf, August 2015, 18 pages.

[37] PAZY, A. Semigroups of linear operators and applications to partial differential equations, vol. 44 of Applied Mathematical Sciences. Springer-Verlag, New York, 1983.

[38] Renardy, M., And Rogers, R. C. An introduction to partial differential equations, second ed., vol. 13 of Texts in Applied Mathematics. Springer-Verlag, New York, 2004.

[39] Sabanis, S. A note on tamed Euler approximations. Electron. Commun. Probab. 18 (2013), no. $47,1-10$.

[40] Sabanis, S. Euler approximations with varying coefficients: the case of superlinearly growing diffusion coefficients. arXiv:1308.1796 (2015), 24 pages.

[41] Song, M. H., Lu, Y. L., And Liu, M. Z. Convergence of the tamed Euler scheme for stochastic differential equations with piecewise continuous arguments under non-Lipschitz continuous coefficients. arXiv:1502.00058 (2015), 16 pages.

[42] SzPruch, L., And Zhāng, X. $V$-integrability, asymptotic stability and comparison theorem of explicit numerical schemes for SDEs. arXiv:1310.0785 (2015), 33 pages.

[43] Tambue, A., And Mukam, J. D. Strong convergence of the tamed and the semi-tamed Euler schemes for stochastic differential equations with jumps under non-global Lipschitz condition. arXiv:1510.04729 (2015), 35 pages.

[44] Tretyakov, M. V., And Zhang, Z. A fundamental mean-square convergence theorem for SDEs with locally Lipschitz coefficients and its applications. SIAM J. Numer. Anal. 51, 6 (2013), 3135-3162.

[45] Wang, X., And Gan, S. The tamed Milstein method for commutative stochastic differential equations with non-globally Lipschitz continuous coefficients. J. Difference Equ. Appl. 19, 3 (2013), 466-490. 
[46] Zhang, Z. New explicit balanced schemes for SDEs with locally Lipschitz coefficients. arXiv:1402.3708 (2014), 15 pages.

[47] Zong, X., Wu, F., And Huang, C. Convergence and stability of the semi-tamed Euler scheme for stochastic differential equations with non-Lipschitz continuous coefficients. Appl. Math. Comput. 228 (2014), 240 - 250. 